Galvanically Isolated, Wide-Band Current Sensors

\title{
Galvanically Isolated Wide-Band Current Sensors
}

\author{
Dissertation by \\ Fani Liakou
}

Dissertation Committee

Prof. Georgios Stamoulis, supervisor

Prof. Elias N.Houstis

Prof. Ioannis Moondanos 
Galvanically Isolated, Wide-Band Current Sensors 


\section{Acknowledgments}

First of all, I would like to thank the person who is responsible for most of the work that has been done, Dr. Panos Dimitropoulos. I would like to express my gratitude for all the knowledge and experience that I gained all these years.

I would like to thank my supervisor, Prof. George Stamoulis for the support and for making this dissertation possible. Also, I would like to thank the other two members of the dissertation committee, prof. Elias Houstis and prof. Ioannis Moondanos and all the other 4 members for the study of this thesis (prof. E. Hristoforou, prof. A. Birbas, prof. N. Eumorfopoulos and prof. P. Bozanis).

I would like to thank my friends from LMIS3 lab at EPFL for the help and support during the period that I was a visitor there and especially Dr. Mirjana Banjevic and Dr. Beat Furrer.

For the technical support I would like to mainly thank Jsyr and K. Daloukas.

Last but not least, I would like to thank my parents mainly because they always say 'If you have decided to do that, we are with you'. Also, I thank my favorite aunt Eva for the long period that I had to stay in Athens. 
Galvanically Isolated, Wide-Band Current Sensors

This work is partially funded by Marie Curie FP7 IAPP IHACS project. 
Galvanically Isolated, Wide-Band Current Sensors

This dissertation is dedicated to:

My family and

To all those who stand by me 


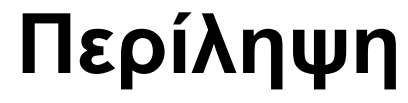

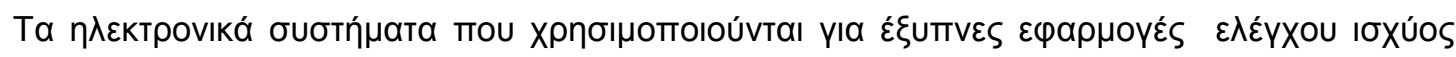

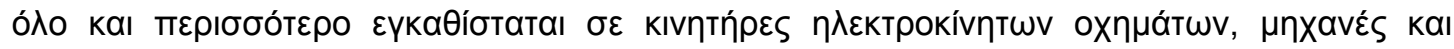

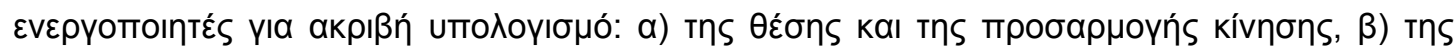

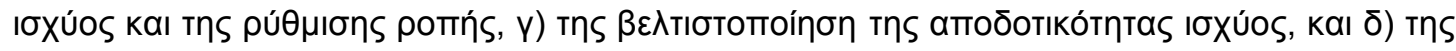

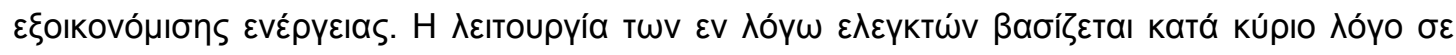

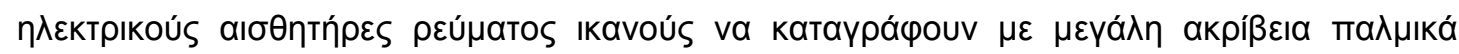

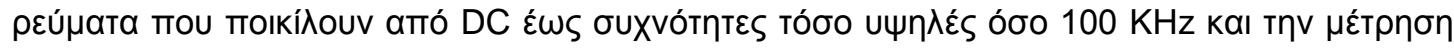

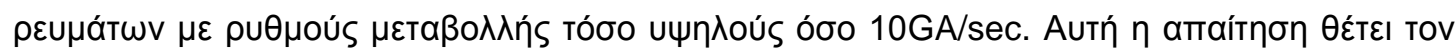

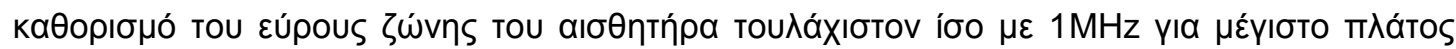

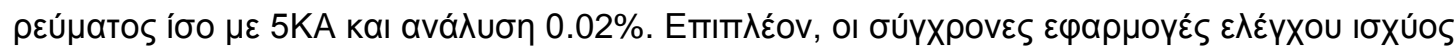

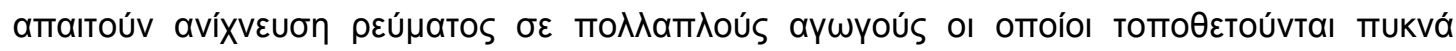

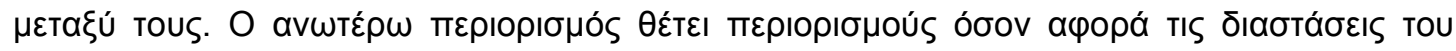

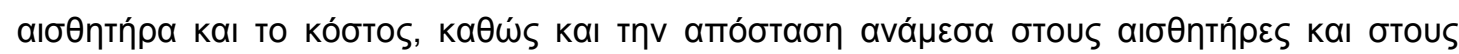

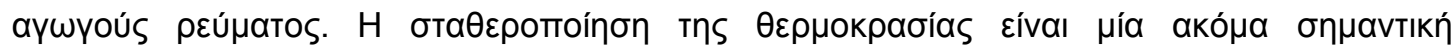

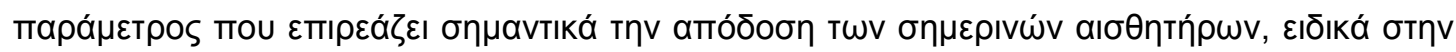

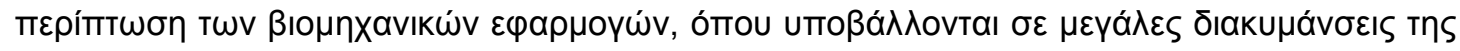
$\theta \varepsilon \rho \mu о к р а б і ́ \alpha$ s.

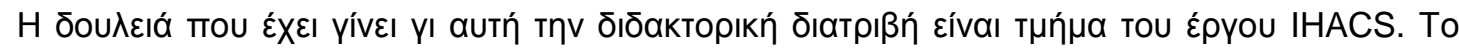

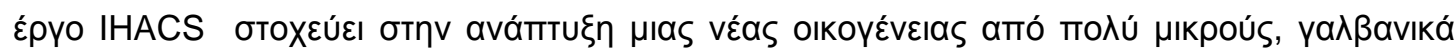

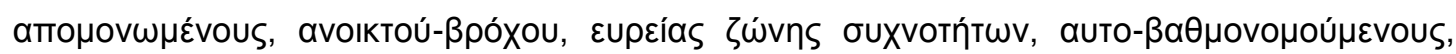

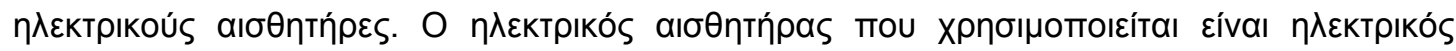

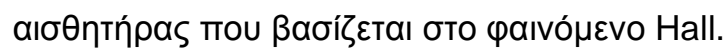

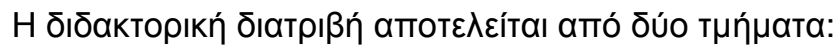

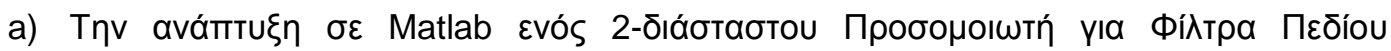

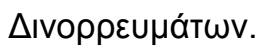

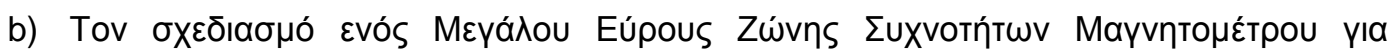

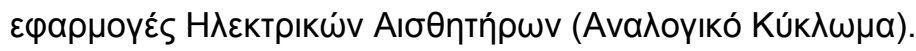

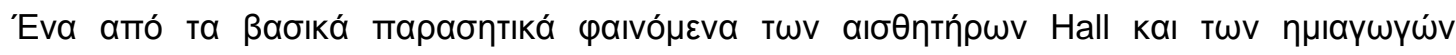

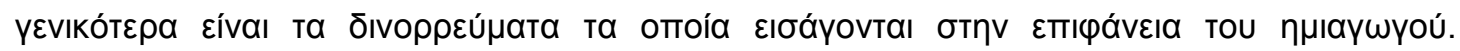

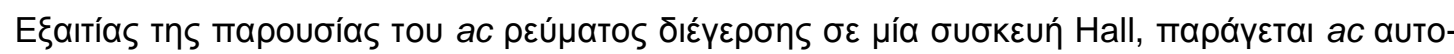

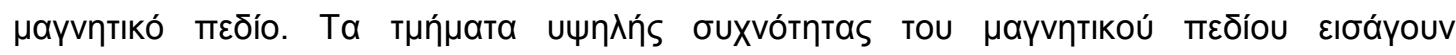

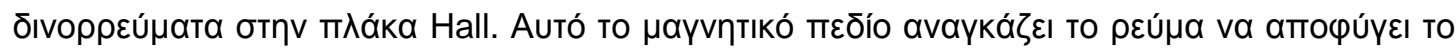

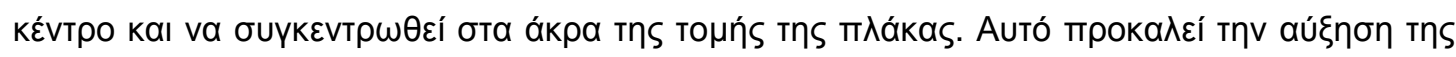

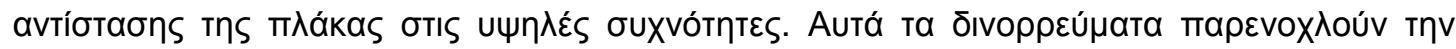

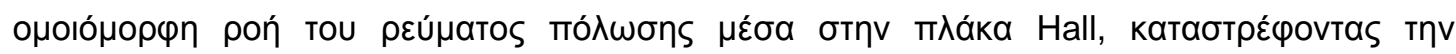




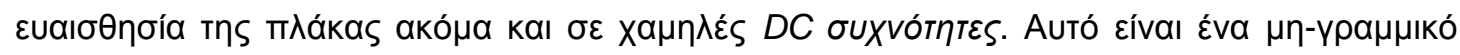

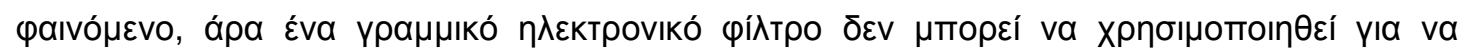

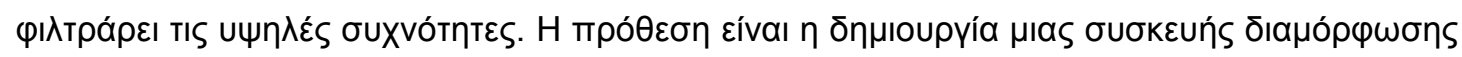

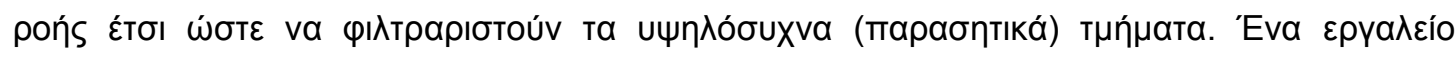

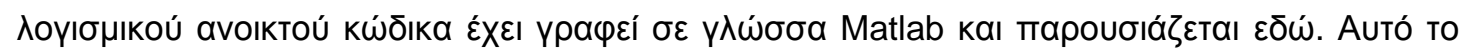

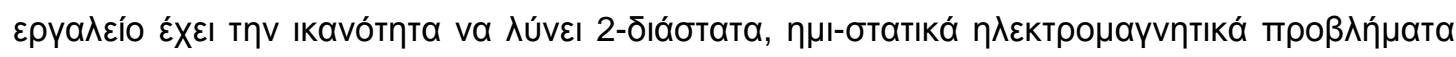

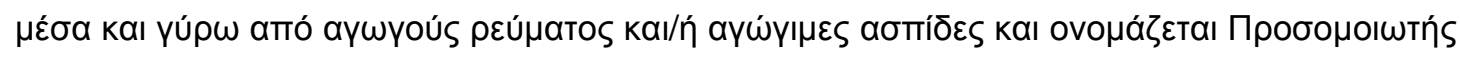

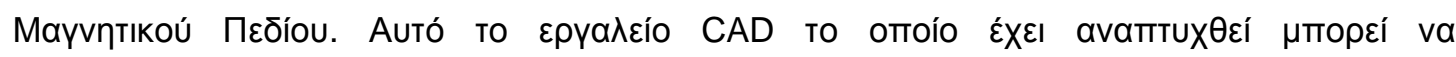

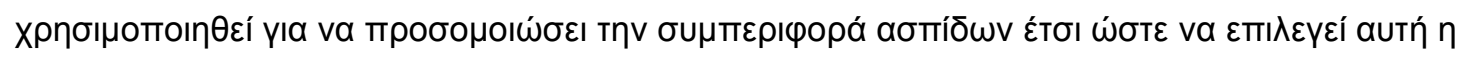

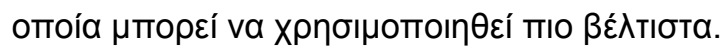

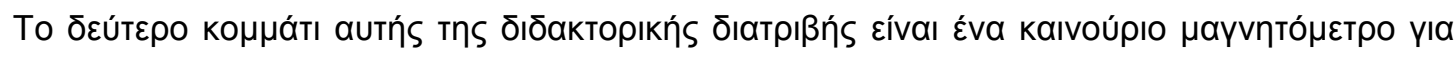

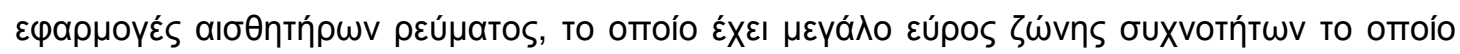

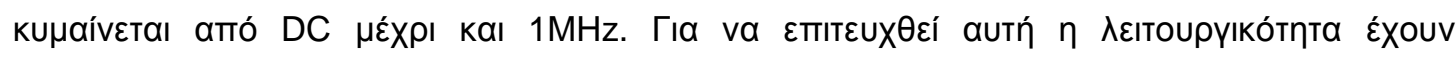

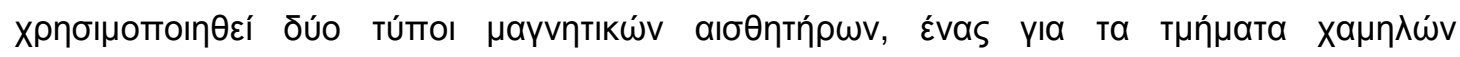

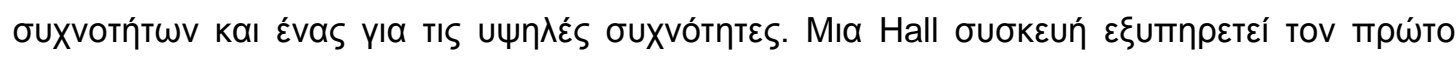

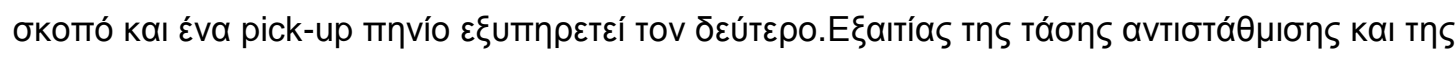

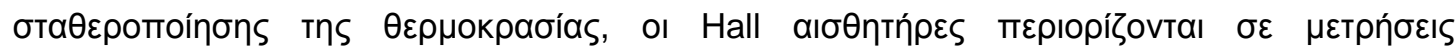

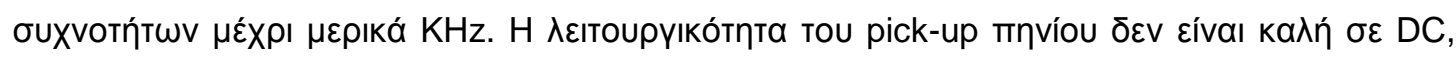

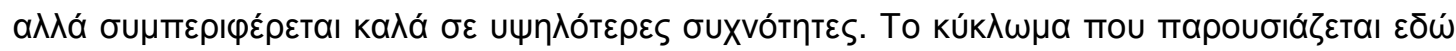

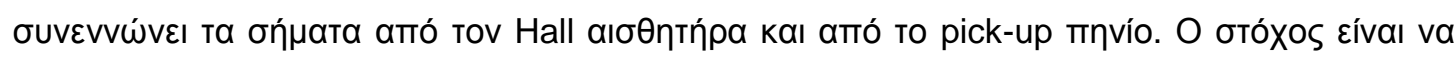

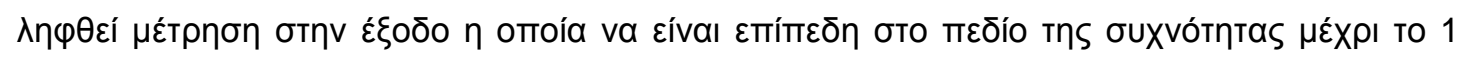

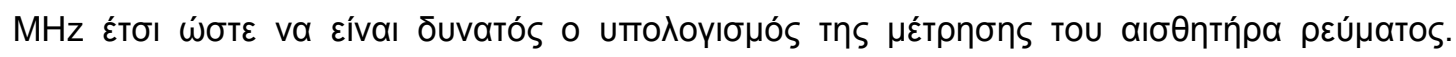

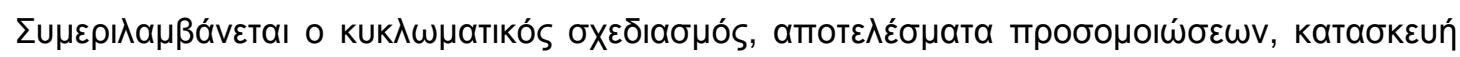

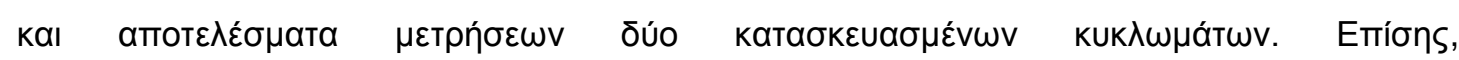

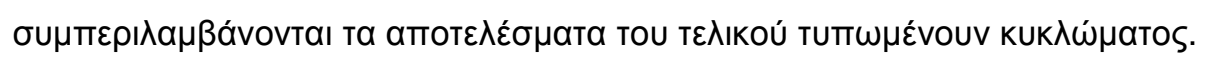




\section{Abstract}

Electronic systems for smart power control are being increasingly installed in modern electrically-propelled vehicles, motors, and actuators for precise: a) position and motion adjustment, b) force and torque regulation, c) power efficiency optimization, and d) energy saving. The functionality of such controllers relies primarily on electric current sensors capable of monitoring precisely pulsed currents varying from DC up to frequencies as high as $100 \mathrm{KHz}$ and of reading current alteration rates as high as $10 \mathrm{GA} / \mathrm{sec}$. This requirement sets the sensor bandwidth specification to at least $1 \mathrm{MHz}$ for $5 \mathrm{KA}$ maximum current amplitude and resolution of $0.02 \%$. Additionally, modern power control applications require current sensing in multiple conductors positioned densely among each other. The aforementioned restriction poses limitations concerning sensor dimensions and cost as well as positioning distance between sensor and current conductors. Temperature stability is another important parameter that severely affects the performance of current sensors, especially in the case of industrial applications, where they are subjected to large temperature variations.

The work that has been done for this thesis is a part of the IHACS project. The IHACS project aims at the development of a new family of very compact, galvanically isolated, open-loop, wide-band, self-calibrating current sensors. The current sensor that is used is a current sensor based on the Hall effect.

This thesis consists of two parts:

a) The development of 2D Matlab Simulator for Eddy Current Field Filters

b) The design of a Wide-Band Magnetometer for Current Sensing Applications (Readout circuit)

One of the main parasitic effects in Hall sensors and generally in semiconductors are the Eddy Currents that are induced into the semiconductor surface. Because of the presence of ac excitation current in a Hall device, ac self-magnetic field is produced. High frequency magnetic field components induce eddy currents in the Hall plate. This magnetic field forces the current to avoid the center of the device and to concentrate at the extremities of the crosssection of the device. This produces an increase in the device resistance in high frequencies. These Eddy Currents disturb the uniform flow of the biasing current within the Hall plate, destructing the sensitivity of the device even as low frequencies as $D C$. This is a non-linear effect, thus a linear electronic filter cannot be used to filter out high frequencies. The intention is the development of a flux shaping device in order to filter out high frequency (parasitic) field components. An open source software tool written in Matlab script language is developed and presented here. That tool is capable of solving 2D, quasi-static electromagnetic problems inside and around current conductors and/or conducting shields and is called Magnetic Field 
Galvanically Isolated, Wide-Band Current Sensors

Simulator (MFS). This CAD tool that has been developed can be used to simulate the behaviour of shields in order to choose the one that can be optimally used.

The second part of this thesis is a new magnetometer for current sensing applications, with wide bandwidth that ranges from $\mathrm{DC}$ up to $1 \mathrm{MHz}$. In order to achieve this functionality two types of magnetic sensors are used, one capturing low frequency components and one for high frequencies. A Hall device serves the first purpose and a pick-up coil serves the second. Due to offset voltage and temperature stability, Hall sensors are limited to measurements up to few $\mathrm{KHz}$. Pick-up coil performance is not good at DC, but is performing well at higher frequencies. The Readout circuit that is presented here merges the signal from the Hall sensor and the signal from the pick-up coil. The goal is to get a measurement at the output that is flat in the frequency domain up to $1 \mathrm{MHz}$ in order to be able to calculate the current sensor measurement. This work includes the circuit design, simulation results, fabrication and measurement results of two fabricated chips. Also, the results of the final PCB measurements are included. 
Galvanically Isolated, Wide-Band Current Sensors 
Galvanically Isolated, Wide-Band Current Sensors

\section{Table of Contents}

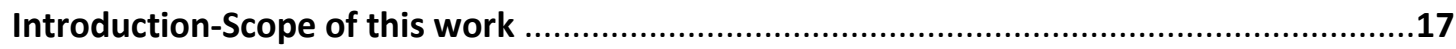

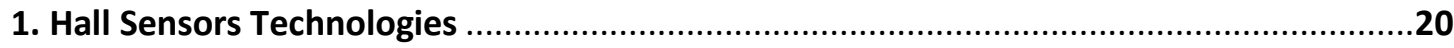

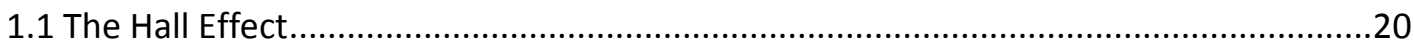

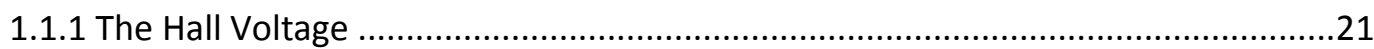

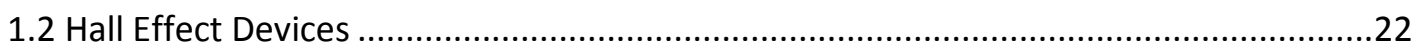

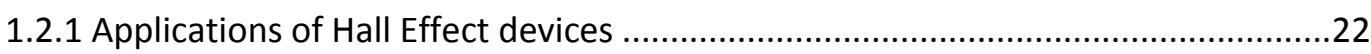

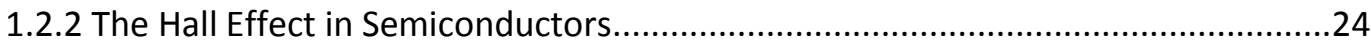

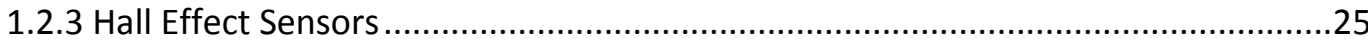

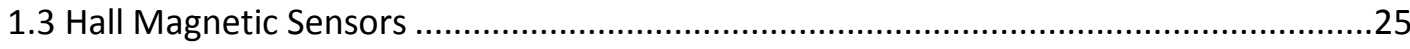

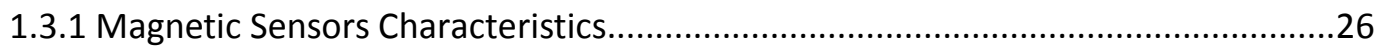

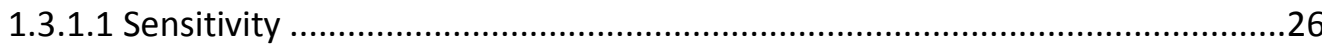

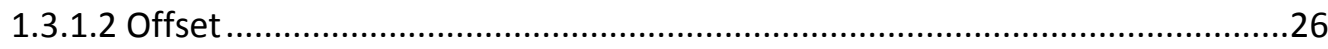

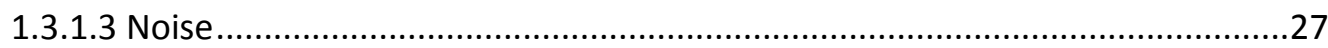

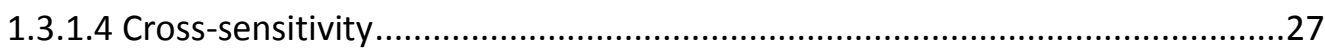

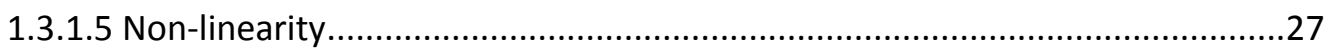

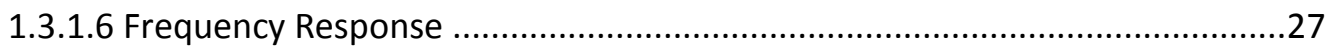

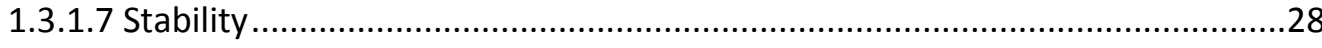

1.4 Applications of Magnetic Hall Effect Sensing Devices ..................................................28

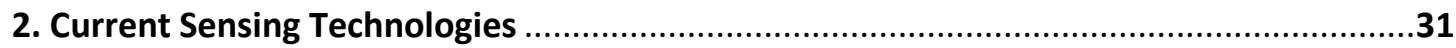

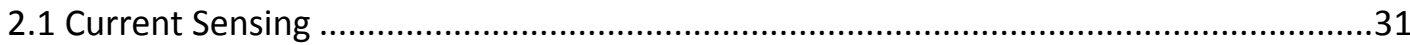

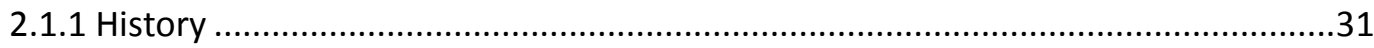

2.2 Overview of Integratable Current Sensor Technologies ..............................................32

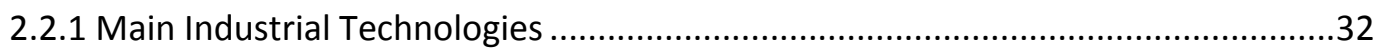

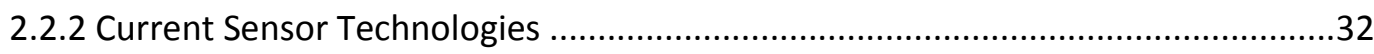

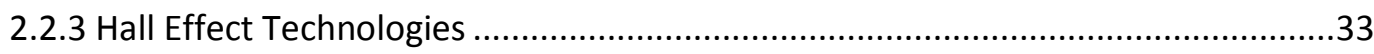

2.2.3.1 Hall Effect Open Loop Current Transducers ....................................................33

2.3.3.2 Hall Effect Closed-Loop Current Transducers...................................................36

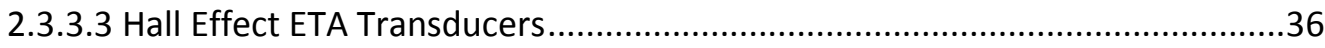

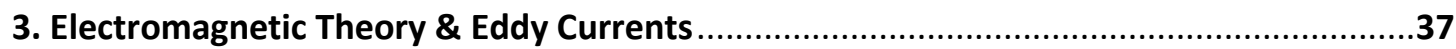

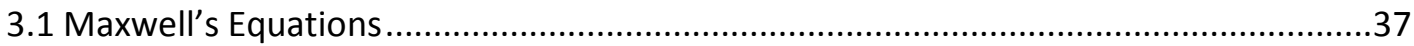

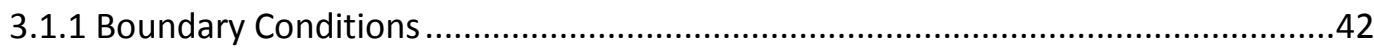

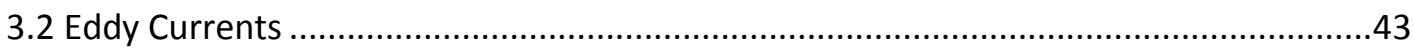

3.2.1 Main Principle 
Galvanically Isolated, Wide-Band Current Sensors

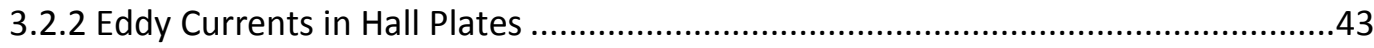

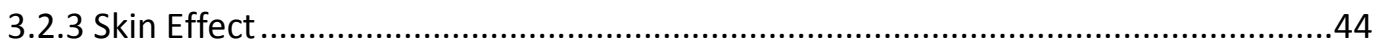

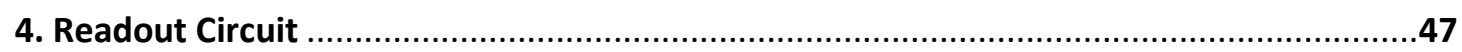

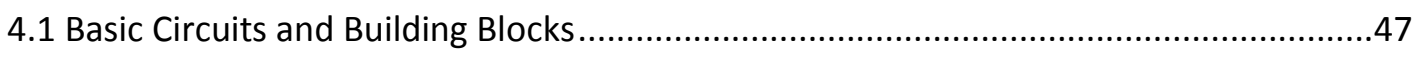

4.1.1 The CMOS Differential Cell (Basic Transconductance) ............................................47

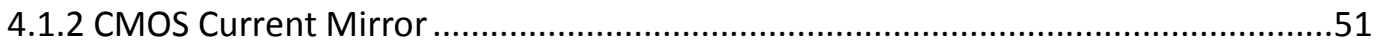

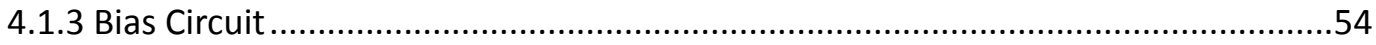

4.1.4 Operational Transconductance Amplifier ..........................................................55

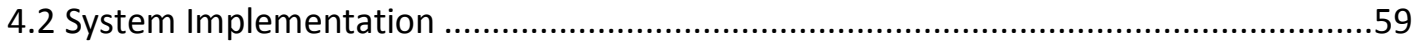

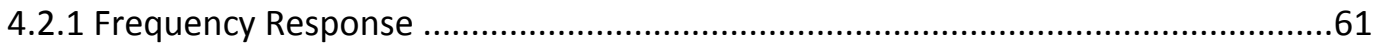

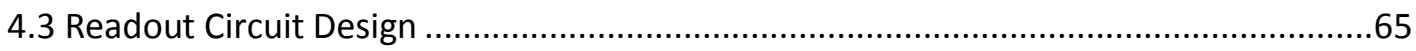

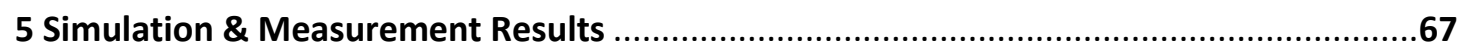

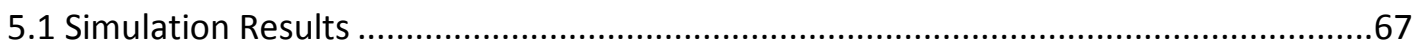

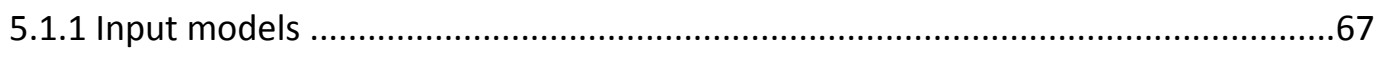

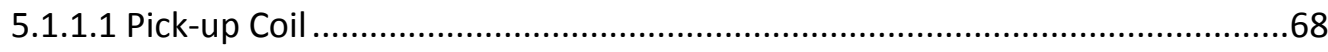

5.1.1.2 Hall Sensor - Magnetometer .....................................................................68

5.1.2 Simulation Results with modeled inputs..............................................................69

5.1.3 Simulation Results with the Hall sensor circuit ..................................................76

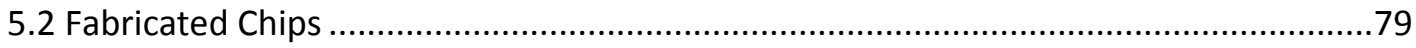

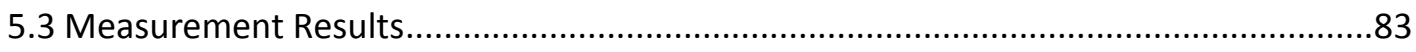

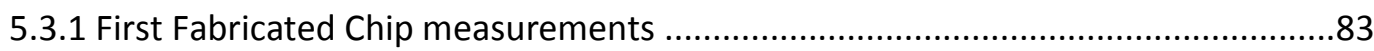

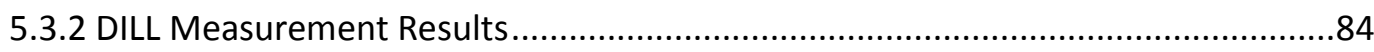

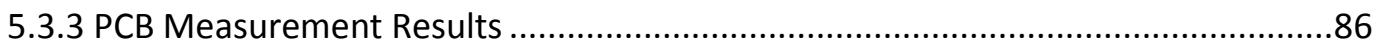

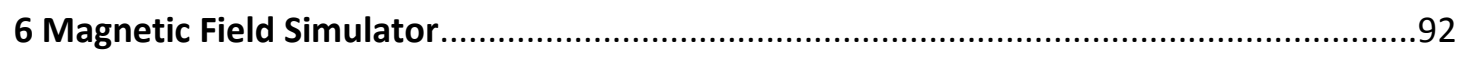

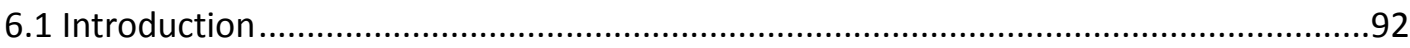

6.2 Theory

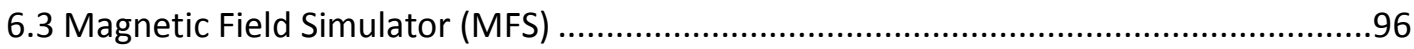

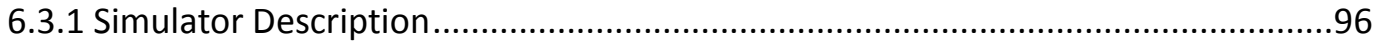

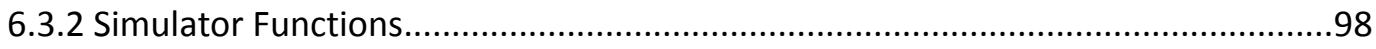

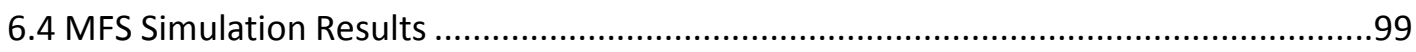


Galvanically Isolated, Wide-Band Current Sensors

\section{List of Figures}

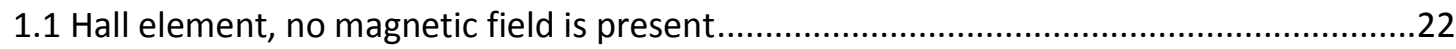

1.2 Hall element, perpendicular magnetic field is present ...................................................22

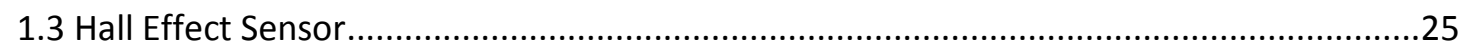

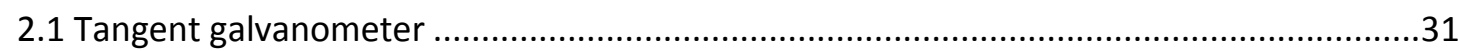

2.2 Basic configuration of a Rogowski current transducer .....................................................34

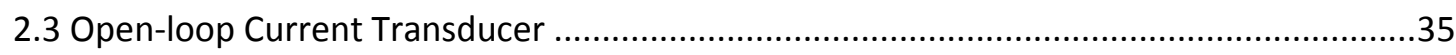

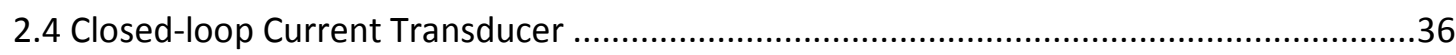

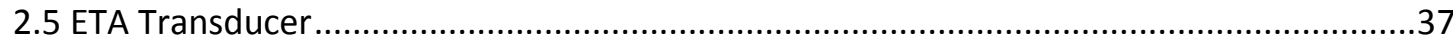

3.1 Hall plates placed above and below current currying conductor .....................................43

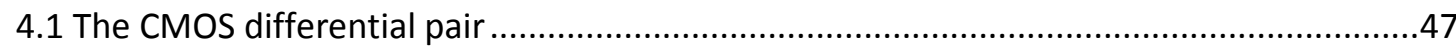

4.2 Actual implementation of the Basic Transconductance Circuit ........................................50

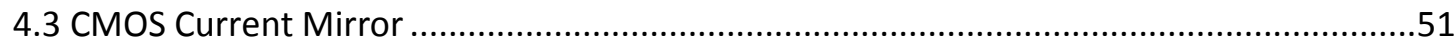

4.4 Actual Implementation of the CMOS Current Mirror .......................................................53

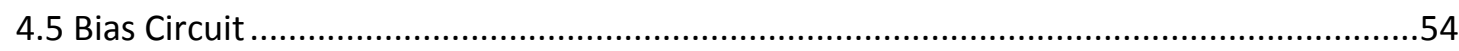

4.6 Operational Transconductance Amplifier (OTA) .......................................................55

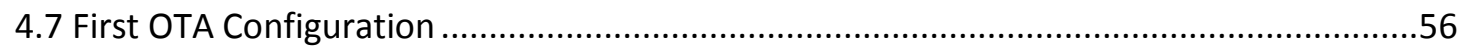

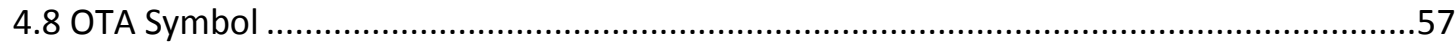

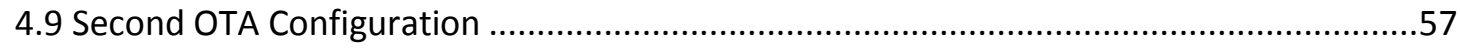

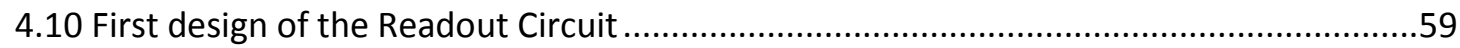

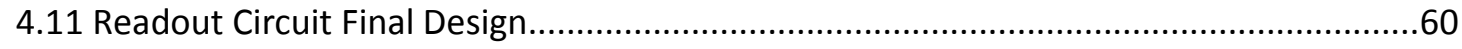

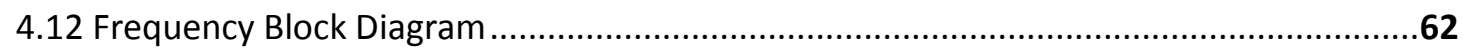

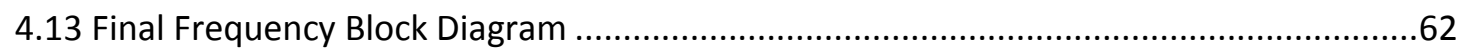

4.14 Actual implementation of the Frequency Block Diagram ................................................63

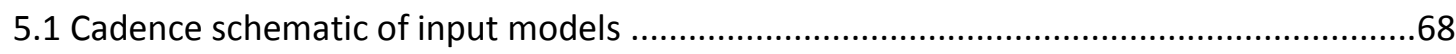

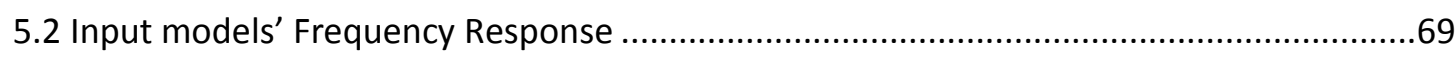

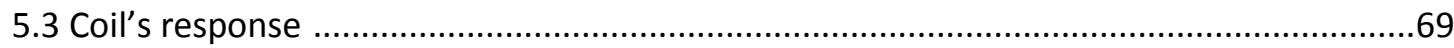

5.4 Input voltages and output current frequency response for varying cap values .................70

5.5 Output voltage frequency response for varying cap values............................................71

5.6 Input voltages and output current frequency response for varying amplitude values .....71

5.7 Output voltage frequency response for varying amplitude values....................................72

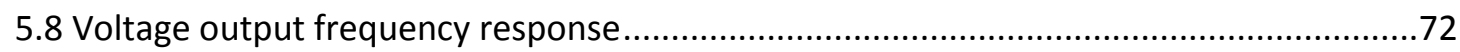

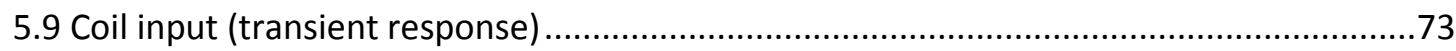

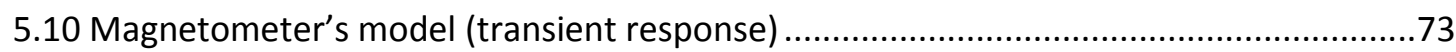


Galvanically Isolated, Wide-Band Current Sensors

5.11 Output current transient response (low frequencies values) .........................................74

5.12 Output current transient response (high frequencies values) ......................................74

5.13 Output voltage transient response (low frequencies values) ..........................................75

5.14 Output voltage transient response (high frequencies values) ........................................75

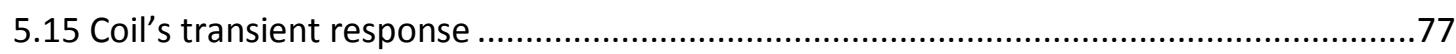

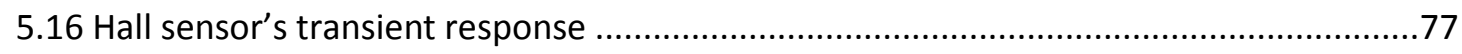

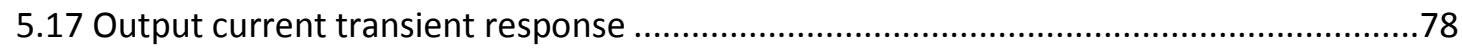

5.18 Magnetometer's model (transient response) ….............................................................78

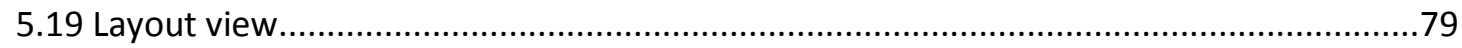

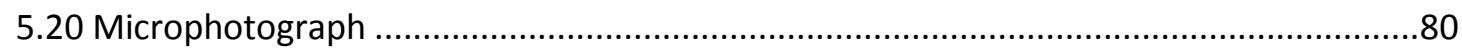

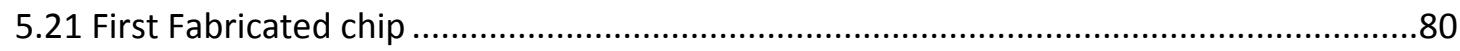

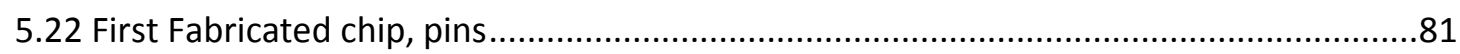

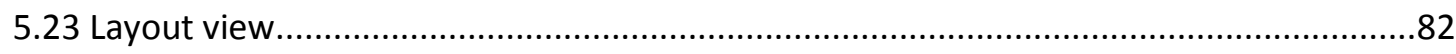

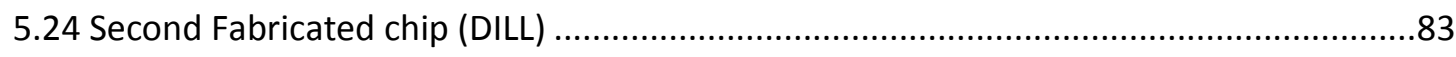

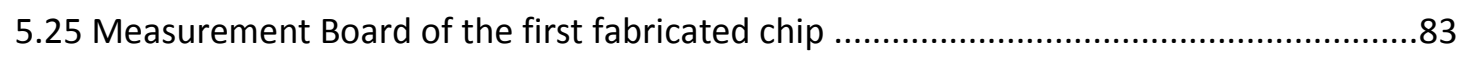

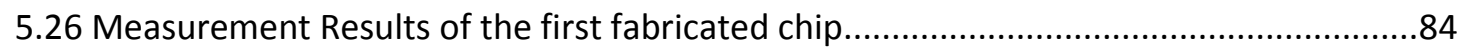

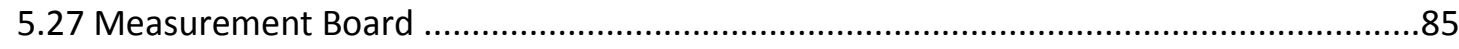

5.28 Measurement Results of the second fabricated chip ....................................................86

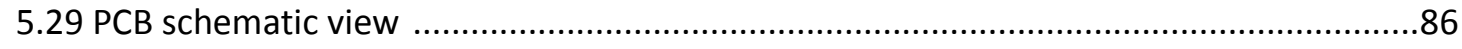

5.30 PCB

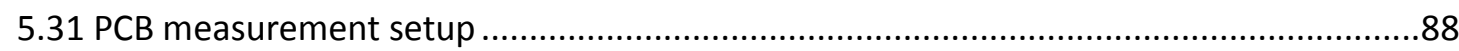

5.32 PCB measurement results, with flat coil at the first input ...........................................8

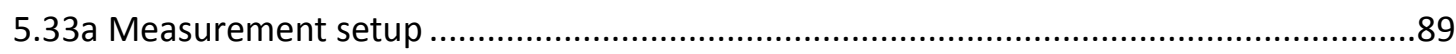

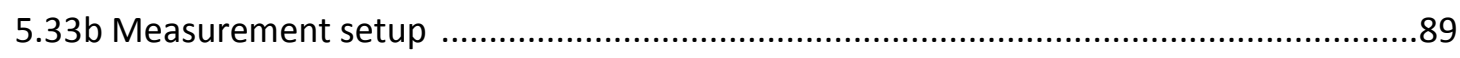

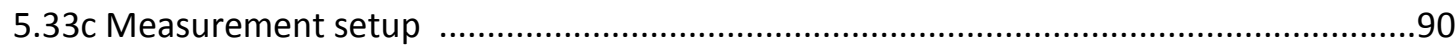

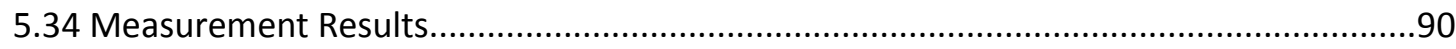

6.1 Primitive implementation of a galvanically isolated current sensing structure.................92

6.2 Current sensor incorporating a copper ring providing appropriate shielding against high

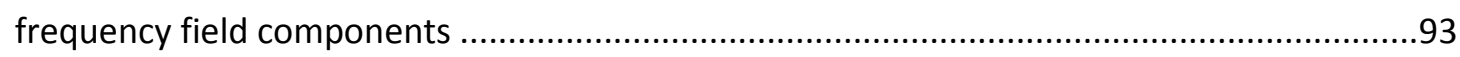

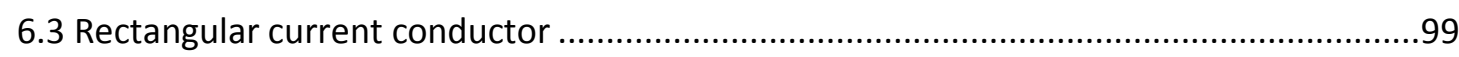

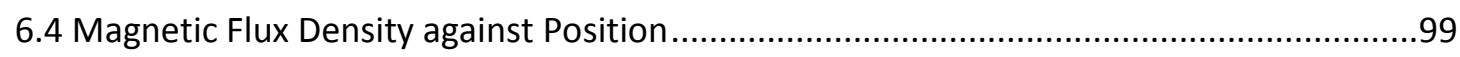

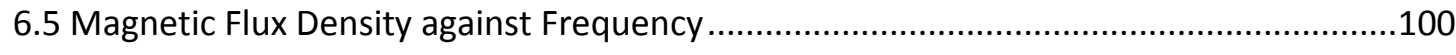

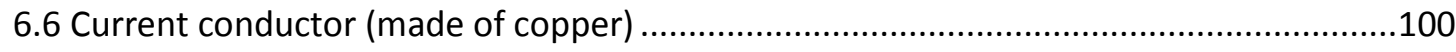

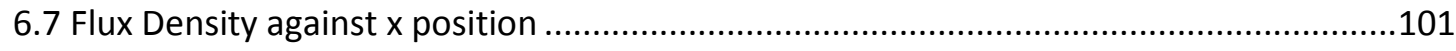


Galvanically Isolated, Wide-Band Current Sensors

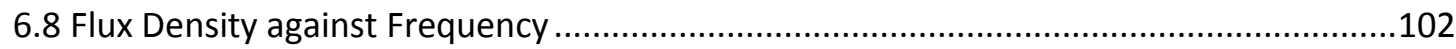

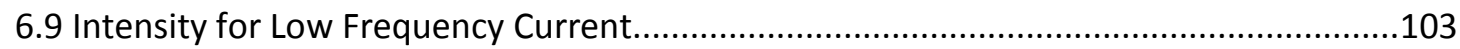

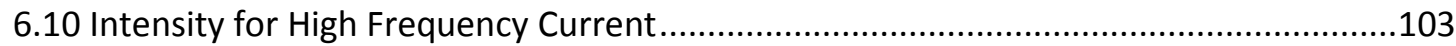

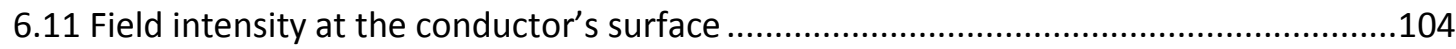

6.12 Flux Density against $x$ position for various frequency values.......................................105

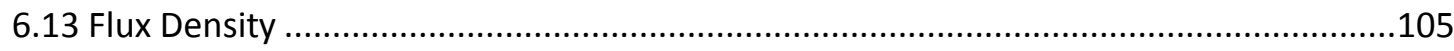


Galvanically Isolated, Wide-Band Current Sensors

\section{List of Tables}

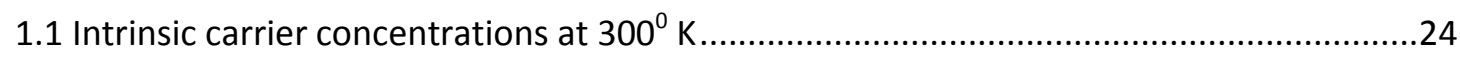

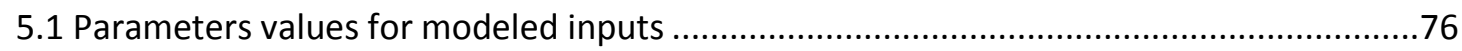

5.2 Parameters values with the Hall sensor at the second input..........................................77 
Galvanically Isolated, Wide-Band Current Sensors 


\section{Introduction - Scope of this work}

This work is a part of the IHACS project. The IHACS project aims at the development of a new family of very compact, galvanically isolated, open-loop, wide-band, self-calibrating current sensors. The sensors are based on sophisticated multi-axes CMOS HALL magnetometers with no ferromagnetic parts that employ an elaborate technique for sensitivity stabilization against temperature effects. Additional mechanical parts will be incorporated that exploit Eddy-Current effects to provide for appropriate field filtering of the magnetic field that is induced by the monitor current conductors. These RF filters comply with the specifications set by the sensitivity-stabilizing subsystem and serve as supports for the CMOS integrated HALL magnetometers. Hence, conceiving a current sensor design with the aforementioned characteristics entails the following technological challenges:

1. A wide-band, CMOS-integrated HALL magnetometer with very large dynamic range and fine resolution is produced and experimentally evaluated in a series of different current sensing applications. The unique performance of this device enables its use in almost any conventional HALL device application, as well as allowing for the deployment of new, innovative sensor systems that were impossible up to now.

2. A sensitivity-stabilizing subsystem for HALL magnetometers should be conceived that provide continues-time sensor calibration.

3. A series of Eddy-Current field filters should be devised and manufactured that provide for appropriate operation of the stabilizing-subsystem in the - rather unlikely - case of mismatch of the properties of employed semiconductor devices that may appear at extreme temperature variations. The shields will also serve as compact mechanical supports for the integrated magnetometers.

IHACS project aims at the development of a current sensor which works at frequencies up to $1 \mathrm{MHz}$. In order to achieve this functionality, two types of magnetic sensors should be used, namely:

- one capturing low frequency components and

- one for high frequencies.

A Hall device serves the first purpose, sensing low frequency field components and a pick-up coil packaged on a printed circuit board serves the second, sensing high frequency field components.

The objective is the development of a sophisticated continuous-time sensitivity calibration system for HALL magnetometers.

In order to give a more thorough description of the project and how these parts are connected together, a simple explanation of each part will be described in the sequel: 
Galvanically Isolated, Wide-Band Current Sensors

1. Development of IHACS magnetometers. This part aims at the development of an integrated wide-band HALL magnetometer with large dynamic range that incorporates innovative Analog and Digital VLSI Readout Circuits executing advanced signal processing. This part includes intermediate prototyping and experimental activities as well as the development of an advanced CAD tool for the simulation of the performance of integrated HALL magnetometers. A wide-band HALL magnetometer that is optimized for pulsed-current sensing applications is designed. The magnetometer compromises a combination of appropriately designed HALL sensing devices with innovative readout circuit. The design parameters are:

- The sensor is capable of reading current signals with: nonlinearity less than

$1.5 \%$ over $0-150 \mathrm{~A}$ range, wide dynamic range $0-2.8 \mathrm{kA}$, high slew-rate $14 \mathrm{MA} / \mathrm{s}$ and low temperature cross-sensitivity $500 \mathrm{ppm} /{ }^{\circ} \mathrm{C}$ over $100^{\circ} \mathrm{C}$.

- The sensor can provide close mounting to current currying conductors.

- The magnetometer is integrated in standard CMOS process in order to achieve unique characteristics with regard to dimensions, spatial sensing resolution, performance-cost ratio, reproducibility and production yield.

2. Development of Sensitivity-stabilizing Subsystem. At this part, a sophisticated continues-time sensitivity calibration system for HALL magnetometers is developed. This part includes intermediate prototyping and experimental activities, as well as tasks aiming at the monolithic integration of IHACS magnetometers with the sensitivity-stabilization subsystem. Any mixed-signal and/or digital circuits required by specific IHACS application shall be addressed at this part.

3. Development of Eddy-Current Field Filters. This part addresses tasks focusing on modelling, design, and prototype manufacturing of Eddy-Current shielding devices providing for field filtering. In this part special equipment is designed that allows for thorough experimental testing and evaluation of the field filters.

4. Current Sensor Integration and Calibration. In this part, IHACS magnetometers including sensitivity-stabilising subsystem - are combined with different Eddy-Current Flux-Shaping devices to form a family of current sensors. The sensors have been tested in different applications, by means of special equipment that are tailor-made for this purpose.

The IHACS parts that are developed and are included in this thesis are the Development of Eddy-Current Field Filters and the Readout Circuit.

A CAD tool written in Matlab script language has been developed in order to simulate different shields and choose the one that may be optimally used in order to filter out parasitic field components. 
Galvanically Isolated, Wide-Band Current Sensors

A Readout Circuit has been developed, which merges the low frequency components of the HALL sensor and the high frequency components of the pick-up coil. This leads to a functionality of the system up to $1 \mathrm{MHz}$. 


\section{Hall Sensors Technologies}

\subsection{The Hall Effect}

The Hall effect is named after the American Physicist Edwin $\mathrm{H}$ Hall, who discovered it in 1879.A graduate student, Hall was attempting to prove that a magnet directly affects current and not a wire bearing the current, as was believed at that time. His experimental device was a gold leaf mounted on a glass plate. An electric current was passing through the leaf and a sensitive galvanometer was connected across the leaf at two nearly equipotential points. The leaf was placed between the poles of an electromagnet. Experimenting with this topology, Hall discovered a new action of the magnet on electric currents. This action is now called the Hall effect.

In order to understand the Hall effect, one must understand how charged particles, such as electrons, move in response to electric and magnetic fields. In the case of the electric field, a charge will experience a force in the direction of the field, which is proportional both to the magnitude of the charge and the strength of the field. This effect is what causes an electric current to flow. Electrons in a conductor are pulled along by the electric field developed by differences in potential (voltage) at different points. In the case of the magnetic field, a charged particle doesn't experience any force unless it is moving. When it is so, the force experienced by a charged particle is a function of its charge, the direction in which it is moving and the orientation of the magnetic field in which it is moving through [1]. It should be noticed that particles with opposite charges will experience force in opposite directions.

What Hall actually observed was an electromotive force in the leaf, acting at right angles to the primary electromotive force and the magnetic field. It appeared as if the electric current was pressed but not moved, towards one side of the leaf. Hall concluded that the new electromotive force was proportional to the product of the intensity of the magnetic field and the velocity of the electricity. This conclusion can be expressed by equation (1.1).

$$
E_{H} \propto[u \times B]
$$

Where $E_{H}$ is the Hall electric field, $u$ is the carrier velocity and $B$ is the magnetic induction.

The carrier velocity can be expressed by equation (1.2)

$$
u=\mu \cdot \mathrm{E}_{\mathrm{e}}
$$

Where $\mu$ presents the carrier mobility and $E_{e}$ is the applied external electric field.

The Hall effect comes as a manifestation of the action of the Lorenz force on quasi-free charge carriers. The Lorenz force is the force acting on a charged particle in electromagnetic field. A charged particle will be accelerated in the same linear orientation as the $\boldsymbol{E}$ field, but it will curve perpendicularly to both the charge velocity $u$ and the magnetic field $\boldsymbol{B}$, according to the right hand rule. The existence of the Lorenz force is the fundamental indication of the 
Galvanically Isolated, Wide-Band Current Sensors

presence of electric and/or magnetic fields. The Lorenz force can be expressed by equation (1.3).

$$
F=e \cdot E+e[u \times B]
$$

Where $\mathrm{F}$ is the Lorenz force, e is the carrier charge, $\boldsymbol{E}$ is the electric field, $\mathrm{u}$ is the electron or hole velocity and $\boldsymbol{B}$ is the magnetic induction [1].

Assuming that there are no space charges equation (1.4) can be derived

$$
J=\sigma \cdot \mathrm{E}+\mu_{\mathrm{H}}[J \times B]
$$

Where $J$ is the current density, $\sigma$ is the carrier conductivity and $\mu_{H}$ is the Hall carrier mobility.

The Hall mobility can be calculated using equation (1.5)

$$
\mu_{\mathrm{H}}=r_{H} \cdot \mu
$$

Where $\mu$ is the carrier drift mobility and $\mathrm{r}_{H}$ is the Hall factor [3].

There is another way that Hall effect appears, besides the generation of the Hall electric field. A magnetic field introduces an angle between the total electric field and the current density vectors. This is so, because in the presence of a magnetic field, the total electric field $E=E_{e}+E_{H}$ is not collinear with the external electric field $E_{e}$. Thus, an angle exists between the electric fields and is expressed by equation (1.6)

$$
\tan \Theta_{\mathrm{H}}=\left|E_{H}\right| /\left|E_{e}\right|
$$

This angle is called the Hall angle and can also be calculated by equation (1.7)

$$
\Theta_{\mathrm{H}} \approx \arctan (\mu \mathrm{B})
$$

At weak magnetic induction, the Hall angle can be expressed by equation (1.8)

$$
\Theta_{\mathrm{H}} \approx(\mu \mathrm{B})
$$

Over the last hundred years, the Hall effect has proved to be quite useful in the study of metals, semiconductors and magnetic materials.

\subsubsection{The Hall Voltage}

Nowadays, Hall's experiment can be done using a Hall element which can be a thin sheet of a semiconductor. A current passes through this sheet and output connections are placed perpendicular to the direction of the current. When no magnetic field is present, current distribution is uniform and no potential difference is seen across the output, as it is presented in Figure 1.1 [6]. 


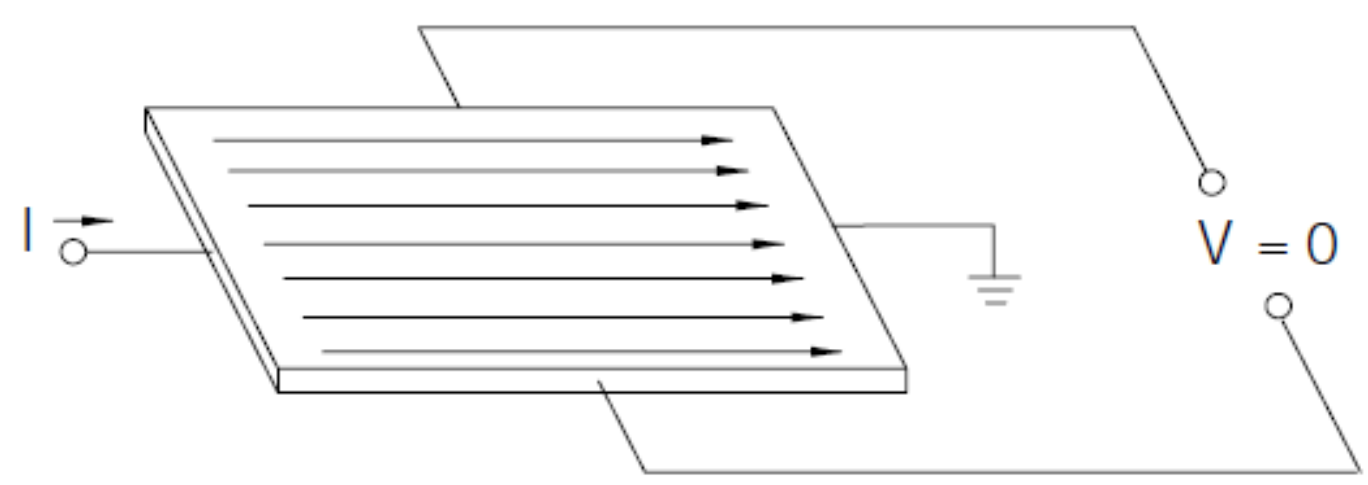

Figure 1.1: Hall element, no magnetic field is present

When a perpendicular magnetic field is present, the Lorenz force acts on the carriers and disturbs the current distribution resulting in a voltage across the output, as it is presented in Figure 1.2 [6]. This voltage that is measured is the Hall voltage and is proportional to the vector cross product of the current $I$ and the magnetic field $\boldsymbol{B}$.

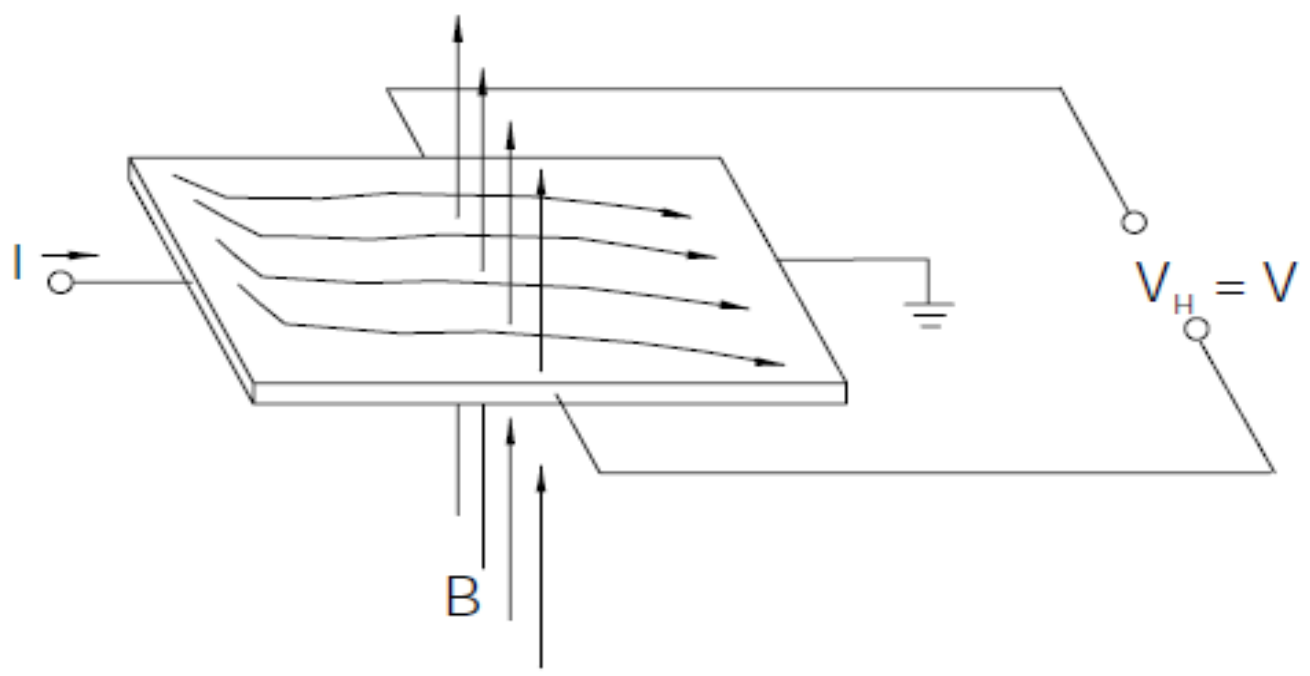

Figure 1.2: Hall element, perpendicular magnetic field is present

Thus, if a Hall cross-section is placed in a static magnetic field $\boldsymbol{B}=\boldsymbol{B z}$, the Hall voltage appears between the sense contacts. The Hall voltage and can be calculated by equation (1.9).

$$
V_{H}=S_{I} \cdot I \cdot B
$$

Where $I$ is the biasing current and $S$ is the current related sensitivity. Current sensitivity can be calculated using equation (1.10).

$$
S_{I}=\frac{G}{n \cdot e \cdot t}
$$


Where $G$ is a magneto-geometrical factor, dependent on the carrier mobility and on the length and the width of the device, $n$ is the carriers' density, $e$ is the carrier charge and $t$ is the thickness of the device [6]. The magneto-geometrical factor is expressed by equation (1.11).

$$
G=\frac{V_{H}}{V_{H \infty}}
$$

Where $V_{H}$ is the Hall voltage of an actual device, and $V_{H \infty}$ is the Hall voltage of a corresponding infinitely long or point-contact device.

By inspection of equations (1.9) and (1.10), it follows that the Hall voltage is inversely proportional to the carriers' density. For this reason, it is not usually practical to make Hall effect devices using most metals.

\subsection{Hall Effect Devices}

The term Hall Effect devices is used in order to describe all solid-state electron devices whose principle of operation is based on the Hall effect. Hall devices which are similar to the one that Hall had used in order to discover this effect, today are called Hall Plates. If the shape of the Hall device is undefined, the terms Hall device, Hall element or Hall sell are used in order to refer to these devices. Some Hall plates take after their name according to their shape, like Hall cross or Hall junction. For example, a combination of two Hall crosses is called Hall bridge. When the device is used as a sensor, usually the term Hall sensor is used. Another category is referring to integrated circuits, incorporating a combination of a Hall device with some electronic circuitry. These integrated circuits are usually called Hall ASIC (application-specific integrated circuit) or Hall IC.

\subsubsection{Applications of Hall Effect devices}

Hall effect devices are mainly used in two applications, as magnetic sensors and as a means of characterizing material. The Hall voltage of a Hall device can be considered as a signal that is carrying information. If the material properties, the device geometry and the biasing conditions are known, the Hall voltage can give information regarding the magnetic induction $\boldsymbol{B}$. In this case, the Hall device can be used as a magnetic sensor. On the other hand, consider the fact that the biasing conditions and the magnetic induction of a Hall device with a known geometry are controlled. In this case, from the measured Hall voltage, important properties of the material that the device is made of can be concluded. In this case, the Hall device is used as a means of characterizing material.

The development of semiconductor technology caused the sensor applications of Hall effect devices to become important. This is so, because the Hall effect is strong enough when semiconductor material are used. Therefore, the first Hall effect magnetic sensors became 
commercially available in the mid-1950s. This is a few years after the discovery of highmobility compound semiconductors.

\subsubsection{The Hall Effect in Semiconductors}

In order to improve the Hall effect, materials should be found that do not have as many carriers per unit volume as metals do. These materials should have lower carrier density and thus exhibit the Hall effect more strongly for a given current and depth. Those materials are semiconductor materials such as silicon, germanium, and gallium-arsenide that provide the low carrier densities that are needed to realize practical Hall elements. In the case of semiconductors, carrier density is usually referred to as carrier concentration. In Table 1.1, the carrier concentration of various materials is presented [2]

\begin{tabular}{|c|c|}
\hline Material & Carrier Concentration $\mathbf{( c m}^{-3}$ ) \\
\hline Copper & $8.4 \times 10^{22}$ \\
\hline Silicon & $1.4 \times 10^{10}$ \\
\hline Germanium & $2.1 \times 10^{12}$ \\
\hline Gallium-Arsenide & $1.1 \times 10^{7}$ \\
\hline
\end{tabular}

Table 1.1: Intrinsic carrier concentrations at $300^{\circ} \mathrm{K}$

By inspection of Table 1.1, can be realized that these semiconductors material have carrier concentrations that are orders of magnitude lower than those that are found in metals. This is so, because in metals most atoms contribute a conduction electron. On the other hand, electrons in semiconductors become available for conduction only when they acquire enough thermal energy to reach a conduction state. This fact makes the carrier concentration highly dependent on temperature [2].

Nevertheless, semiconductor materials are rarely used in their pure form. Usually, semiconductors are doped with materials in order to raise their carrier concentration to a desired level. In order to make Hall effect devices, there are several advantages of using doped semiconductor materials. The first advantage comes from the fact that the low intrinsic carrier concentrations of pure semiconductors will be doped anyway. In this case, it will be unknown with what or to what degree those materials will be doped. The second advantage of using doped materials is that this allows the choice of the dominant charge carriers. In metals, electrons are the default charge carriers. There, no choice regarding the carriers can be made. On the other hand, in semiconductors there is the choice between electrons and holes. So, there is the choice between $\mathrm{N}$-type material in which electrons are the majority carriers and P-type material in which current is carried by holes. It is noticed that for pure 
Galvanically Isolated, Wide-Band Current Sensors

semiconductors the carrier concentration is a strong function of temperature. In doped semiconductors the carrier concentration is resulting from the addition of dopants and it is mainly a function of the dopant concentration, which does not depend on the temperature.

\subsubsection{Hall Effect Sensors}

Hall effect sensors consist basically of a thin piece of rectangular p-type semiconductor material. When the device is placed within a magnetic field, the magnetic flux lines cause a force on the semiconductor material which deflects the charge carriers (electrons and holes), to either side of the semiconductor. This movement of charge carriers is a result of the magnetic force that they experience while passing through the semiconductor material. As these electrons and holes move sideward a potential difference is produced between the two sides of the semiconductor material. Thus, the movement of electrons through the semiconductor material is affected by the presence of an external magnetic field. This effect is presented in Figure 1.3.

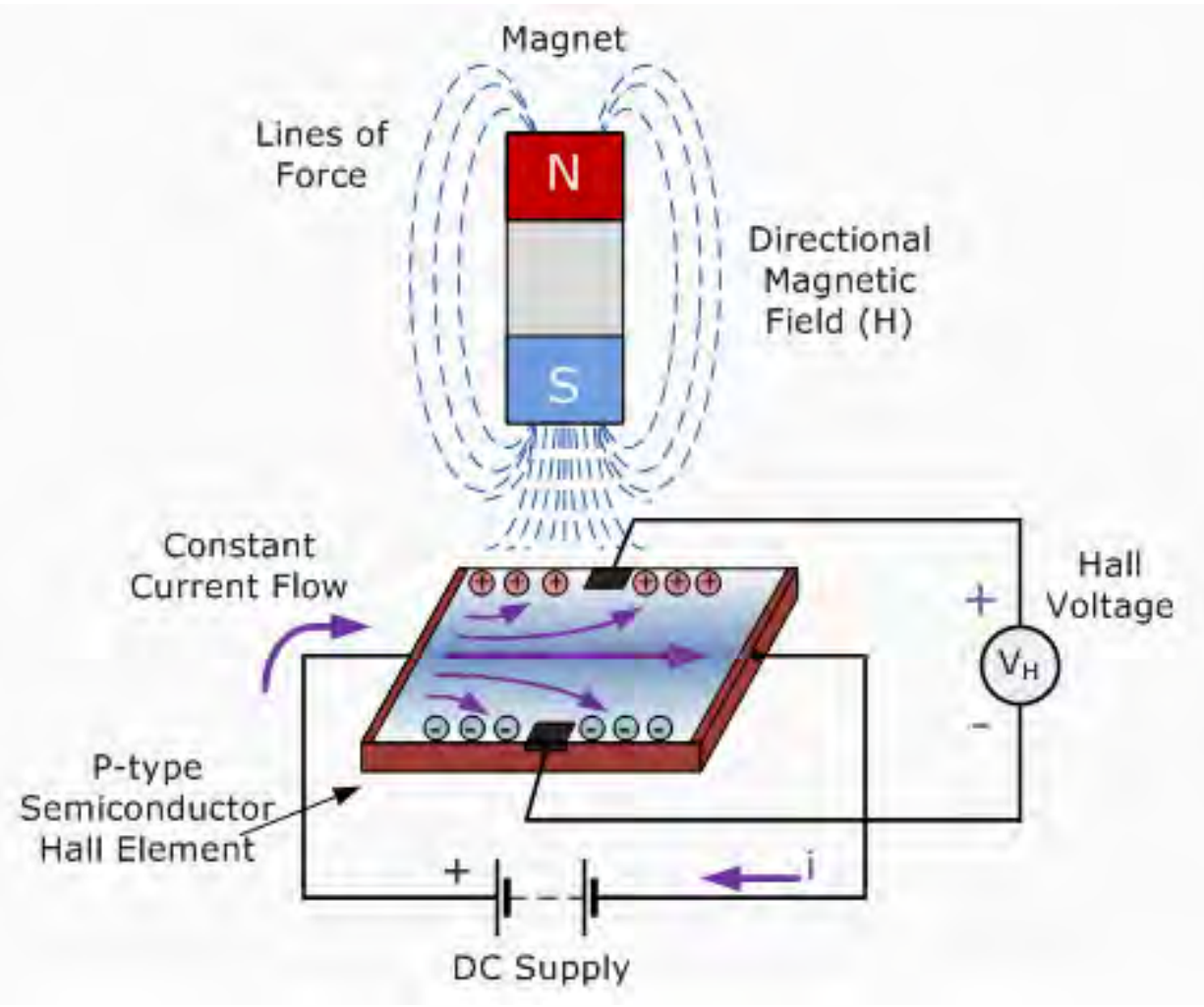

Figure 1.3: Hall Effect Sensor

\subsection{Hall Magnetic Sensors}

Magnetic sensors are devices that convert a magnetic field into an electrical signal. A Hall device can be used as a magnetic sensor, based on the classical Hall effect. A Hall effect sensor is a transducer that varies its output voltage in response to a magnetic field. Hall magnetic sensors are widely used because these sensors are cheap and are compatible with 
Galvanically Isolated, Wide-Band Current Sensors

low-cost integrated circuit technologies. Nowadays, Hall magnetic sensors are the most widely used magnetic sensors. They are not only used to measure a magnetic field, but also they can be applied for linear position, angular position, velocity, rotation and current. Hall magnetic sensors are used in various products like cars and disc drives in personal computers [7].

Hall magnetic sensors encounter some basic characteristics. These characteristics are sensitivity, offset, noise, cross-sensitivity, non-linearity, frequency response and stability.

\subsubsection{Magnetic Sensors Characteristics}

\subsubsection{Sensitivity}

Sensitivity in Hall sensors can be described by explaining the two main sensitivity coefficients, which are current-related sensitivity and voltage-related sensitivity. Current-related sensitivity is described by equation (1.9). Note that the $n \cdot t$ product denotes the surface charge carrier density in a homogeneous plate. The product $n \cdot t \cdot e$ equals the charge of free electrons per unit area of the plate. The best option is to choose low-doped, small doping gradient layers. Such layers are usually used in magnetic sensors. Otherwise, the operation is done with average quantities. The voltage-related sensitivity is denoted in equation (1.11)

$$
S_{V}=G \frac{w}{l}
$$

Where $G$ is the magneto-geometrical factor and $w / l$ is the width to length ratio of the equivalent rectangle. Voltage-related sensitivity increases with a decrease in the effective device length.

\subsubsection{Offset}

Magnetic field offset appears due to the offset output voltage of a Hall magnetic sensor. All Hall plates show an output voltage referred to as offset even when the magnetic field is absent, due to imperfections in the device and other transduction effects other than the Hall effect. Generally, the offset equivalent field is decreased as the Hall mobility is increased. The major causes of offset in Hall plates fabricated with silicon bipolar integrated circuit fabrication process are errors in geometry. The errors are due to etching randomness and rotation alignment [8]. Offset of a silicon Hall plate can be seriously affected by mechanical stress referring to piezoresistance effect $[9,10]$. The piezoresistance effect is the main cause of offset.

The offset causes the output voltage of a Hall plate to be the sum of the Hall voltage and an offset voltage. The offset voltage consists of a number of current-dependent terms and a selfinduced magnetic field. CMOS compatible elements and the spinning current method are used [11], in order to considerably reduce the offset of the output signal. 


\subsubsection{Noise}

There is noise voltage at the output terminal of a Hall device. This noise puts a limit in the measurement precision of the device. The noise in a Hall device is due to thermal noise, generation-recombination noise and 1/f noise. A convenient way to describe the noise properties of a magnetic sensor is in terms of resolution, which is also called detection-limit [2]. A high resolution can be achieved in a large Hall device made of high-mobility material and low $1 / f$ noise parameter when it operates at high power level.

\subsubsection{Cross-sensitivity}

The cross-sensitivity of a magnetic sensor is its undesirable sensitivity to environmental parameters, such as temperature and pressure. For example, there could be temperature coefficient or also temperature coefficient of the voltage-related sensitivity. There are published techniques that are used in order to reduce cross-sensitivity of magnetic sensors [12].

\subsubsection{Non-linearity}

A variety of non-linearity exists in Hall magnetic sensors. These are due to current-related sensitivity, material non-linearity, geometrical non-linearity and non-linearity due to the junction field effect. The current-related non-linearity will appear in a magnetic sensor if its sensitivity depends on magnetic field. The junction field effect non-linearity depends on the device structure, on the biasing conditions and the magnetic field [13].

A technique that is proposed and used in order to compensate geometrical non-linearity is by loading the sensor output with a proper valued resistor. A drawback of this approach is that due to the very large non-linear temperature dependence on the resistivity of the Hall plate materials, it is extremely difficult to keep constant the ratio of the sensor output resistance and the load resistance. The material non-linearity and the geometrical non-linearity exhibit the same quadratic magnetic induction dependence, but this dependence has opposite signs. Someone can take advantage of this fact by cancelling out these two non-linearity effects. Non-linearity due to the junction field-effect can be compensated by adding half the Hall voltage to the junction bias voltage [14].

\subsubsection{Frequency Response}

The frequency response to a magnetic field is frequency dependent due to parasitic effects. One of these parasitic effects is the relaxation time limit, which limits the Hall effect up to $1 \mathrm{THz}$. The main frequency limitation is due to inductive effects. An ac excitation current of a Hall plate produces a self-field, which may generate an inductive signal in the output circuit of the Hall plate. Also, an external ac magnetic field can produce inductive currents or voltages 
Galvanically Isolated, Wide-Band Current Sensors

in both input and output circuits of a device. The main parasitic effects are skin effect and eddy currents. Because of the presence of ac excitation current in a Hall device, ac selfmagnetic field is produced. This self-magnetic field forces the current to avoid the center of the device and to concentrate at the extremities of the cross-section of the device. This produces an increase in the device resistance in high frequencies. Also, if a Hall device is exposed to a fast-varying magnetic field, eddy currents will be induced in the device. Eddy currents have two main effects to the measurement of high-frequency magnetic fields. First, the Eddy currents tend to oppose the change in the field which induces them, which results in phase shift and decrease of the magnetic field acting on the Hall device. Second, in cooperation with the self magnetic field of a Hall plate, eddy currents produce an additional Hall effect. By designing very careful wiring of the device, in the case of high-mobility and high-current Hall devices, this might work well up to frequencies higher than $1 \mathrm{MHz}$. But in low-mobility Hall devices, such as silicon devices, the practical frequency limit is around 10 $\mathrm{KHz}[2]$.

\subsubsection{Stability}

Hall magnetic sensors stability depends on the sensitivity of the device. The instability is proportional to the sensitivity. To cope with this problem, the Hall device active region should be isolated from the surroundings. In order to achieve this, a reverse-biased $p-n$ junction can be used instead of a bare or oxidized surface [2].

\subsection{Applications of Magnetic Hall Effect Sensing Devices}

Applications of Magnetic Hall Sensors are presented below, which are divided in categories [6].

Linear output sensor applications are:

- Current Sensing

Disk drives

Variable frequency drives

Motor control protection/indicators

Power supply protection/sensing

- Position Sensing

Pressure diagrams

Flow meters

Damper controls 
Galvanically Isolated, Wide-Band Current Sensors

Brushless DC motors

Wiperless/contactless potentiometers

- Encoded Switches

Rotary encoders

- Voltage Regulators

- Ferrous Metal Detectors (bias Hall)

- Vibration Sensors

- Magnetic Toner Density Detection

- Tachometers 
Galvanically Isolated, Wide-Band Current Sensors

\section{References}

[1] R.S. Popovic, Hall Effect Devices, IOP Publishing, 2004.

[2] Edward Ramsden, Hall-Effect Sensors: Theory and Applications, Elsevier Inc 2006

http://books.google.com/books?id=R8VAjMitH1QC\&printsec=frontcover\&hl=el\&sourc

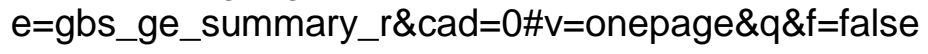

[3] Zoran Randjelovic, Low-Power High Sensitivity Integrated Hall Magnetic Sensor Microsystems, Volume 10.

[4] G. Boero, M. Demierre, P.-A. Besse, R.S. Popovic, Micro-Hall devices:

performance, technologies and applications.

[5] Spinning-current method for offset reduction in silicon Hall plates, Peter Munter

[6] Hall Effect Sensing and Application, Honeywell • MICRO SWITCH Sensing and Control.

[7] R.S. Popovic, Z. Randjelovic, D. Manic, Integrated Hall-effect magnetic sensors.

[8] Bjorklund G 1978 Improved design of Hall plates for integrated circuitry IEEE Trans. Electron Devices ED-25 541-4

[9] Kanda Y and Migitaka M 1976 Effect of mechanical stress on the offset voltage of Hall devices in Si IC Phys. Status Solidi (a) 35 K115-8

[10] Kanda Y and Migitaka M 1976 Design consideration fro Hall devices in Si IC Phys. Status Solidi (a) 38 K41-4

[11] R. Steiner, Ch. Maier, A. Haberli, F.-P. Steiner and H. Batles, Offset reduction in Hall devices by continuous spinning current method, Sensors and Actuators A:Physical, Volume 66, Issues 1-3, 1 April 1998, Pages 167-172

[12] J.C. van der Meer, F.R. Riedijk, P.C. de Jong, E.A. van Kampen, M.J. Meekel, J.H. Huijsing, CMOS quad spinning-current Hall-sensor system for compass application, Sensors 2004, 1434-1437 vol.3

[13] George Caruntu, Ovidiu Dragomirescu, The non-linearity of magnetic sensors, 375-378, Semiconductor Conference 2001

[14] R. S. Popovic and B. Halg, Nonlinearity in Hall devices and its compensation, Solid State Electronics Vol. 31, No.12, 1681-1688, 1988 


\section{Current Sensing Technologies}

\subsection{Current Sensing}

\subsubsection{History}

From the beginnings of electrical engineering the quantification of the basic electrical units was a requirement for the work of physicists and engineers, as well as the basis for the commerce with electrical energy [1]. At first, it was the physical effects directly associated to the moving charge, like the force action of the magnetic field generated by a conductor, Joule's heat or the dissociating action of a current passing through a conductive liquid.

At the end of the $19^{\text {th }}$ century, the currents that were used for technical purposes had reached orders of magnitude that made impossible the direct measurement. In 1837, the tangent galvanometer was first described in a paper. This is a simple compass in the earth's magnetic field influenced by the magnetic field of a current flowing around it, as it is presented in Figure 2.1. In 1884, was reported a measuring shunt made of a copper conductor allowing the extension of the current range up to $200 \mathrm{~A}$ which was considered sufficient at that time. In 1893, currents of more than $10 K A$ were already measured. The instruments, at that point did not have closed magnetic circuits. Thus, the results were affected by stray magnetic fields and there was temperature dependence of the bus bars used as measuring shunts. The first effort to reduce this effect was reported in 1901, where the primary current was only partially linked to the secondary winding in order to reduce the effect of the external magnetic fields [1]. In the 1920s, current transformers for currents up to $50 K A$ and for voltages of up to 250 $K V$ were available.

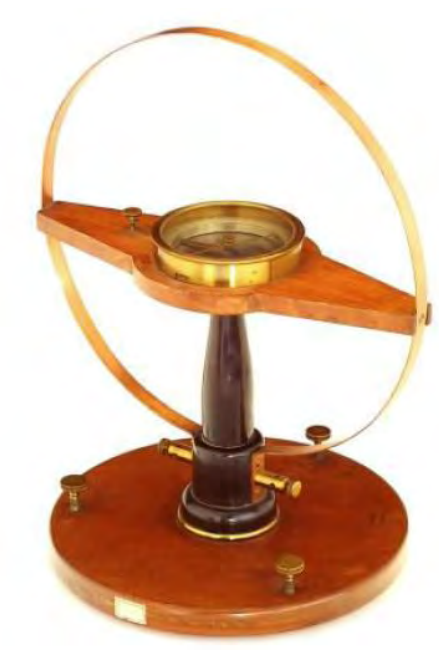

Figure 2.1: Tangent galvanometer

Before 1930, the development of instruments to measure small magnetic fields, the so-called fluxgate magnetometers had begun. Although the Hall-effect was described much earlier, but 
Galvanically Isolated, Wide-Band Current Sensors

it was not able to built practical magnetometers until 1950s. Also, current transducers for large currents came up using Hall cells. In the first transducers the output voltage of a Hall cell in an air-gap was taken directly as a measure for an electric current flowing through the leak of the core. Later a compensation winding was added to achieve smaller errors. The measuring devices that were built at the end of the 1950s for aluminum plants reached errors smaller than $0.1 \%$, which was sufficient for industrial purposes.

\subsection{Overview of Integretable Current Sensor Technologies}

\subsubsection{Main Industrial Technologies}

Today available on the market, there are more than 15 different designs or technologies that can be used for the measurement of the electric current, depending on the specific application requirements, such as peak or rms current, accuracy, bandwidth, environmental robustness or simply cost [2]. Those technologies can be divided into six main categories [1]:

- $A C$ transducers: limited to $A C$ measurements, including the classical current transformers (CTs), Rogowski coil based transducers or a recently developed printed-circuit-board (PCB) technology.

- Hall effect transducers: which can be derived into "Closed-Loop", "Open-Loop" and "ETA technologies".

- Fluxgate transducers: with more than six main designs, each showing specific performance.

- Other field sensing technologies: alternatives to the Hall cells have been deployed into current measurement (e.g. magneto resistance).

- Shunt: referring to shunt based technologies.

- MEMS current transducers: micro-electromechanical systems (MEMS) have been used for current measurement even if none of them is yet at a real industrial stage.

Among these, the isolated technologies often measure the current by sensing the magnetic field it creates with different performances depending on the considered field sensing technologies.

\subsubsection{Current Sensor Technologies}

An overview is given of current sensing technologies that are suitable for packaging into integrated power electronics modules and integrated passive power processing units, such as integrated shunts and integratable Rogowski coils technologies [3]. The technologies that will be considered above are integrated current shunts, current transformers, Rogowski coils, Hall-effect current sensors, Giant Magneto Resistive Sensors and Magneto Impedance sensors. Integrated current sensors aim to achieve some main characteristics which are 
compact size, compatible with manufacturing process, low cost, high bandwidth for high frequency operation, fast response, to introduce low parasitics, high reliability, high noise immunity and high stability with varying module temperature [3].

- Shunt: Although current shunts operate on the principle of the Ohmic voltage drop, practical shunts have intrinsic inductance which limits the accuracy and bandwidth. The main disadvantage of integrated current shunts is that these components lack of galvanic isolation. This has as a result the cost increment and complexity of shunts, in order to achieve isolation. Shunts have low resistance and low temperature coefficient of resistance (TCR). Although they are suitable for $D C$ and $A C$ measurement, are not suitable for applications up to tens of $\mathrm{MHz}$.

- Current transformer: Current transformers have been widely used for $A C$ current sensing at frequencies up to tens of $M H z$. The advantages of this sensing technique are that it provides galvanic isolation and consumes little power. On the other hand, the disadvantages are that the measurement accuracy, the size and the cost can be degraded in some cases and the fact that current transformer introduces additional inductance to the current carrying conductor.

- Rogowski coil: A Rogowski coil is a helix wound around a torus so that the wired ends are joined and is mainly used for isolated current measurements [4]. A basic configuration of a Rogowski current transducer is presented in Figure 2.2. The uniformly wound coil on a nonmagnetic material of constant cross-sectional area is formed into a closed loop. Thus, the voltage induced in the coil is proportional to the rate of change of current. This voltage is integrated, as it is presented in Figure 2.2, producing an output proportional to the current. The advantages of the Rogowski coil use are the capability of measuring large currents (a same sized coil can be used in order to measure $100 \mathrm{~A}$ or $100 \mathrm{KA}$ ), non-saturation, isolation between the testing circuit and the transducer, inexpensive, it can be used easily, has good linearity due to the absence of magnetic materials and is compact and with light weight. The main disadvantage of the current measurements using Rogowski coil is that high bandwidth cannot be achieved by this technique. 


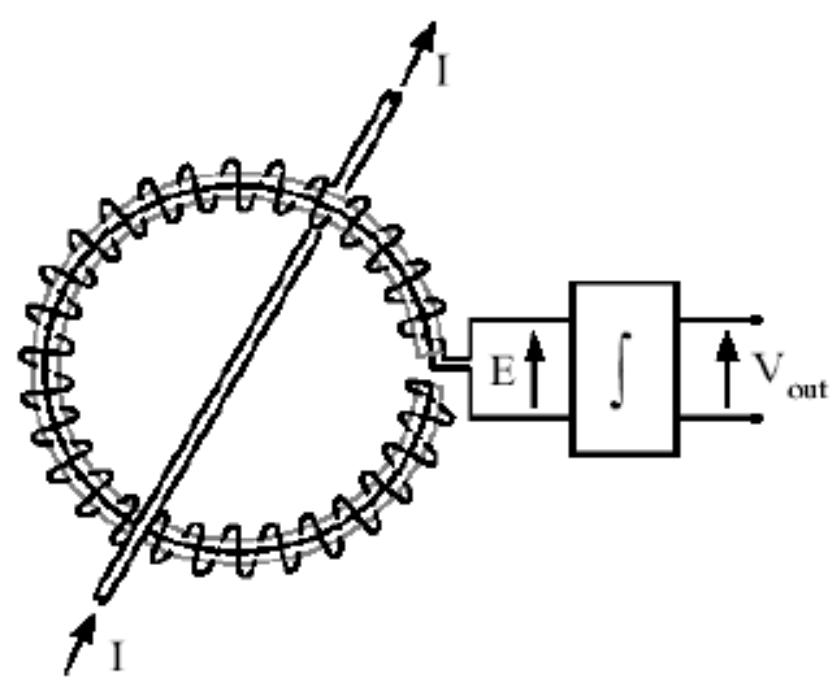

Figure 2.2: Basic configuration of a Rogowski current transducer

- Hall effect sensor: The Hall effect sensor is a magnetic field sensor based on the Hall effect. Hall effect sensors are isolated devices that can be applied to both $A C$ and $D C$ current sensing, up to hundreds of $\mathrm{KHz}$. That limited bandwidth of the Hall sensor is one of its main disadvantages. Due to its simple structure and compatibility with microelectronic devices, a Hall device can be monolithically integrated into a fully integrated magnetic sensor. The main advantages of the Hall effect sensors are that they can be fabricated using a conventional CMOS technology and that they are cheap. The Hall effect sensors mainly operate in closed loop modes for better accuracy and wider dynamic range. The future of the Hall devices will depend on the improvement of sensitivity and reduction of offset.

- GMR current sensor: The Giant Magneto Resistive Sensor (GMR) principle of operation is based on the Giant Magneto resistive (GMR) effect, which is the change of the electrical resistance with the magnetic field. GMR sensors can be effectively used to sense the current by measuring the magnetic field that is generated by the current. The main advantage of this technique is that the GMR sensors can be placed in such a topology that the sensitivity can be improved and the temperature dependent effects can be minimized. The main disadvantage of these sensors is that it is not possible to be integrated and have to be used as an added component.

- MI Current Sensor: The principle of operation of the Magneto Impedance (MI) sensors is based on the Magneto Impedance (MI) effect. The MI effect refers to the variation of the impedance of a magnetic material carrying a low intensity, high frequency $A C$ current when it is subjected to an external magnetic field. The advantages of the use of this current sensing technique are low cost, small size, high sensitivity and wide bandwidth. These advantages provide them with the potential in order to become an alternative to the existing current sensors. The main disadvantage is that the material technology is very complicated.

Today, mainly only the Hall effect and GMR are used for current transducers made in large quantities [1]. 
Galvanically Isolated, Wide-Band Current Sensors

\subsubsection{Hall Effect Technologies}

The three Hall effect based technologies used for AC and DC current measurement are discussed below [1].

\subsubsection{Hall Effect Open Loop Current Transducers}

Open-loop Hall effect transducers use a Hall sensing element placed into the air gap of a magnetic circuit, like the one that is presented in Figure 2.3 [1].

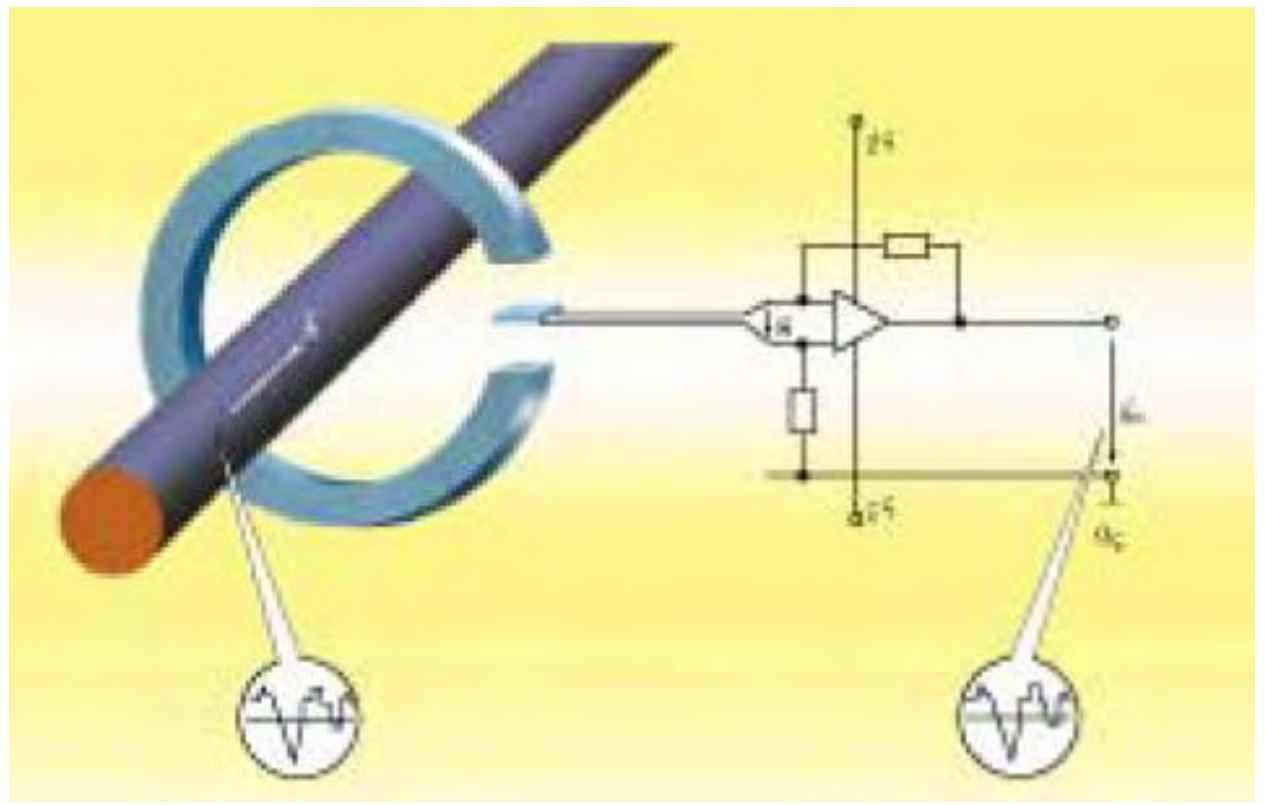

Figure 2.3: Open-loop Current Transducer

The magnetic induction detected by the Hall cell is theoretically proportional to the primary current to be measured. Measurement inaccuracies are mainly due to magnetic and electronic non-linearity, as well as offsets that are created by the Hall cell, the processing electronics and the magnetic circuit hysteresis. The use of a magnetic circuit offers several advantages, like to focus on the field of the Hall cell, to amplify the field magnitude and to shield against external magnetic perturbations.

Open-loop transducers ensure galvanic isolation and are able to measure DC, AC and complex current waveforms. Among their advantages are low power consumption, reduced weight and size, relatively low priced and in general well suited for industrial applications. The main drawbacks of the open-loop transducers are moderate bandwidth and temperature dependence of the measurement accuracy. In specific applications, high frequency eddycurrent loses may be a limiting factor.

Overall accuracy is in general in the range of a few present due to DC offset at zero current, DC magnetic offset, gain and linearity error, amplitude attenuation and phase shift when the 
bandwidth limit is reached and noise. Additional errors are due to temperature variations and offset drift.

\subsubsection{Hall Effect Closed-Loop Current Transducers}

The difference between the open-loop and the closed-loop current transducers is that the later have a built-in compensation circuit which improves performance. The closed-loop current transducer is presented in Figure 2.4. The Hall cell of closed-loop current transducers drive a secondary coil current in a way that the magnetic field in the air gap equals zero. Hall effect closed-loop transducers are ensuring galvanic isolation and are capable of measuring AC, DC and complex current waveforms. Among their advantages are excellent accuracy and linearity, low temperature drift and higher bandwidth (mainly up to $100 \mathrm{KHz}$, and occasionally goes up to $300 \mathrm{KHz}$ ). The main drawbacks of the closed-loop technology are the higher power consumption, the larger dimensions and higher costs compared to simpler open-loop designs. At high frequencies, the measuring performance is set by the current transformer performance.

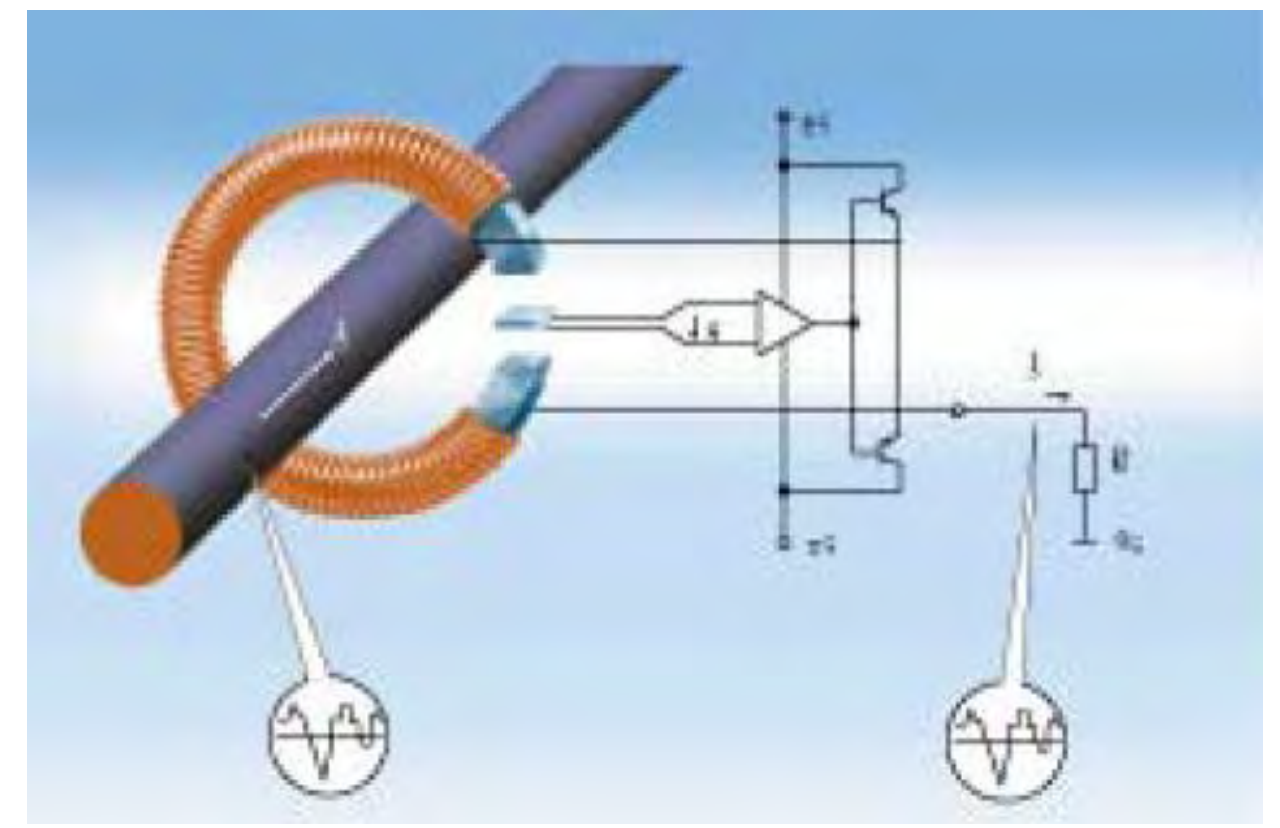

Figure 2.4: Closed-loop Current Transducer

\subsubsection{Hall Effect ETA Transducers}

Hall effect ETA transducers employ a combination of open-loop and closed-loop technologies. At low frequencies $(2-10 \mathrm{KHz})$ they work as open-loop transducers, thus the Hall cell is providing a signal proportional to the primary current to be measured. At high frequencies they work as current transformers. These signals are electronically added in order to form a common output signal, as it is presented in Figure 2.5. ETA transducers provide galvanic isolation and are capable of measuring AC, DC and complex current waveforms. Among their 
Galvanically Isolated, Wide-Band Current Sensors

advantages are high bandwidth (the typically higher frequency limit is $100 \mathrm{KHz}$ ) and low power consumption. Their major drawback is the big size of the magnetic circuit. At low frequencies, ETA technology measurements accuracy has larger sensitivity to temperature changes. Also, the cost for ETA technologies is higher than simpler open-loop designs.

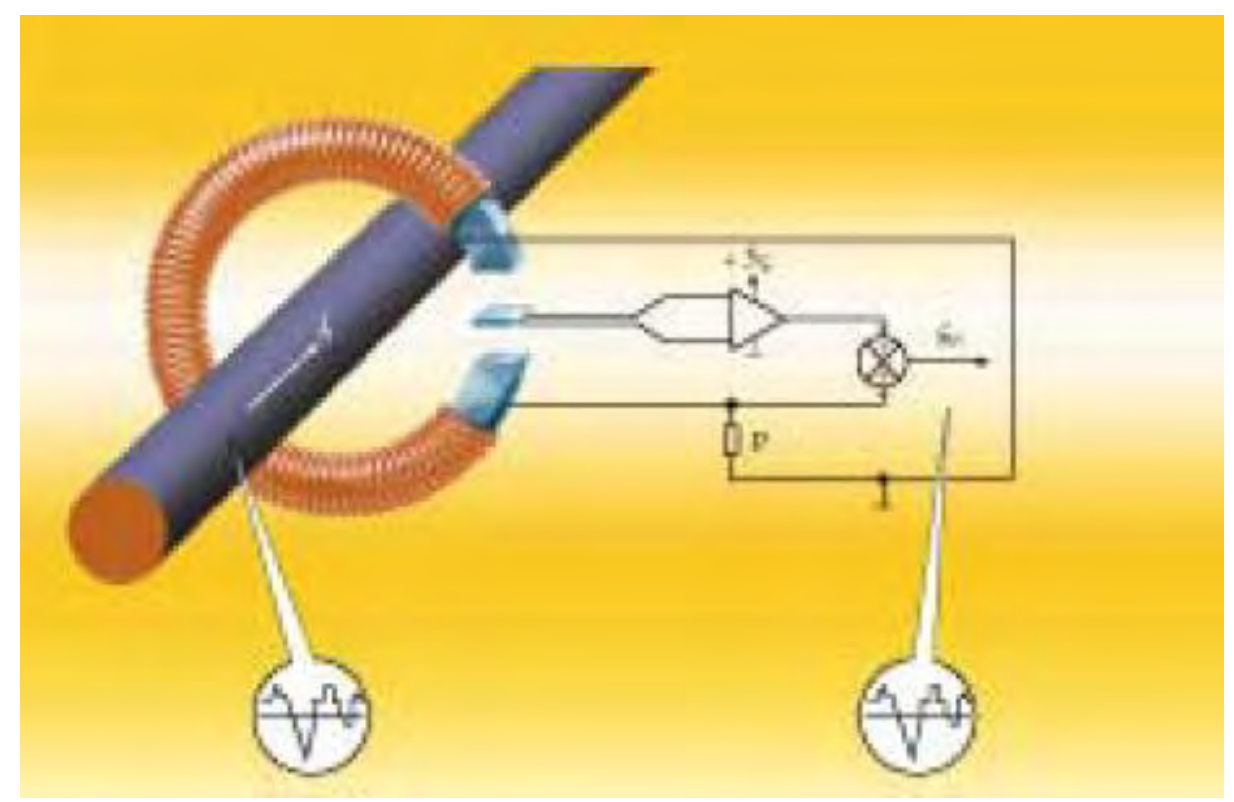

Figure 2.5: ETA Transducer 
Galvanically Isolated, Wide-Band Current Sensors

\section{References}

[1] Eric FAVRE, Wolfram TEPPAN, Current Sensing in Electric Drives, A Future and History Based on Multiple Innovations, LEM Group Switzerland

[2] R. Burkel et al., LEM technology and application brochure, edition 3, May 2004

[3] Chucheng Xiao, Lingyin Zhao, Tadashi Asada, W. G. Odendaal, J. D. van Wyk, An Overview of Integratable Current Sensor Technologies, IEEE Industry Applications Conference, 2003, 1251-1258 vol.2

[4] Nicolas Karrer, Patric Hofer-Noser, A new current measuring principle for power electronic applications, European Conference on Power Electronic and Applications 1999 


\section{Electromagnetic Theory \& Eddy Currents}

\subsection{Maxwell's Equations}

Maxwell's equations are named after the Scottish physicist and mathematician James Clerk Maxwell, since these equations are all found in an early form in a four-part paper which he published between 1861 and 1862. The mathematical form of the Lorentz force law also appeared in this paper.

Maxwell's equations are a set of partial differential equations that, together with the Lorenz force law, form the foundation of classical electrodynamics, classical optics and electric circuits. The former, in turn form the basis for modern electrical and communications technologies.

Faraday's Law can be described by equation (3.1)

$$
|\varepsilon|=-\frac{d \Phi_{\mathrm{B}}}{\mathrm{dt}}
$$

Where $|\varepsilon|$ is the magnitude of the electromotive force and $\Phi_{B}$ is the magnetic flux. An electromotive force is merely a voltage that arises from conductors moving in a magnetic field or from changing magnetic fields [1]. The minus sign is an indication that the electromotive force is in such a direction as to produce a current, whose flux when added to the original flux would reduce the magnitude of the electromotive force. The electromotive force can be defined by equation (3.2)

$$
|\varepsilon|=\oint \vec{E} \cdot d l
$$

where I denotes a closed path. Replacing $\Phi$ in equation (1) and substituting the result in equation (3.2), equation (3.3) is derived

$$
\oint \vec{E} \cdot d l=-\frac{d}{d t} \int_{S} \vec{B} \cdot d \vec{S}
$$

Here, the fingers of our right hand indicate the direction of the closed path, and our thumb indicates the direction of $d S$.

Now, let's consider a stationary path. The magnetic flux is the only time-varying quantity on the right side of equation (3.3). Applying Stoke's theorem to the closed line integral and after calculations, equation (3.4) is derived

$$
\nabla \times \vec{E}=-\frac{\partial \vec{B}}{\partial t}
$$

This is one of Maxwell's equations, written in differential. 
If $\boldsymbol{B}$ is not a function of time, equations (3.3) and (3.4) reduce to the electrostatic equations (3.5) and (3.6)

$$
\begin{aligned}
& \oint \vec{E} \cdot d \vec{l}=0 \\
& \nabla \times E=0
\end{aligned}
$$

Thus, Faraday's experimental law has been used to obtain one of the Maxwell's equations in differential form. According to equation (3.4) a time varying magnetic field produces an electric field.

Ampere's circuital law as it applies to steady magnetic fields is presented in equation (3.7)

$$
\nabla \times \vec{H}=\vec{J}
$$

The magnetostatic field is related to the current that produces it, according to the above equation. In the case of time varying fields, it is found that an additional term must be added. This term is $\partial \vec{D} / \partial t$ and is termed as displacement current density. Displacement current is not an electric current of moving charges, but a time varying electric field. Displacement current has the units of electric current density and it has an associated magnetic field. The idea of displacement current was conceived by Maxwell and added it to the electric current term in Ampere's Circuital Law.

This term acts as though it is an additional current that helps to produce the magnetic field $\boldsymbol{H}$. Thus, equation (3.8) can be derived

$$
\nabla \times \vec{H}=\vec{J}+\frac{\partial \vec{D}}{\partial t}
$$

This is another second one of Maxwell's equations.

The relationship between the $\boldsymbol{H}$ and $\boldsymbol{B}$ fields is given in equation (3.9), where $\mu$ is permeability

$$
\vec{H}=\vec{B} / \mu
$$

A second form of equation (8) can be obtained by applying Stoke's theorem and is derived in (3.10)

$$
\begin{aligned}
& \int_{S}(\nabla \times \vec{H}) \cdot d \vec{S}=\int_{S} \vec{J} \cdot d \vec{S}+\int_{S} \frac{\partial \vec{D}}{\partial t} \cdot d \vec{S} \\
& \oint_{L} \vec{H} \cdot d \vec{l}=I_{s}+\int_{S} \frac{\partial \vec{D}}{\partial t} \cdot d \vec{S}
\end{aligned}
$$

Equation (3.10) can be considered the integral form of equation (3.8) and equation (3.8) can be called the differential form, respectively. Equation (3.10) is the more general form of Ampere's circuital law that applies when a time varying field is present.

One formulation of the relationship between the electrostatic field and the charges that produce it is known as Gauss' Law, which is presented in equation (3.11) 
Galvanically Isolated, Wide-Band Current Sensors

$$
\int_{S} \varepsilon \overrightarrow{\mathrm{E}} \cdot \mathrm{d} \overrightarrow{\mathrm{S}}=\int_{\mathrm{V}} \rho \cdot \mathrm{dV}=\mathrm{Q}
$$

where $Q$ is the total charge enclosed by the surface. The surface integral can be thought of as the outflow or flux of the field $\varepsilon \cdot E$ through the surface $S$. In addition to the electric field $\vec{E}$, a second electric vector $\vec{D}$ is defined by equation (3.12), where $\varepsilon$ is the permittivity of the space.

$$
\vec{D}=\varepsilon \cdot \overrightarrow{\mathrm{E}}
$$

The reason for the definition of this second vector is that permittivity $\varepsilon$ often varies with position. By integration of equation (3.11), equation (3.13) is derived

$$
\int_{S} \vec{D} \cdot d \vec{S}=\int_{V} \rho \cdot \mathrm{dV}
$$

where $\rho$ refers to the total charge. This is the third of Maxwell's equations and remains unchanged from its non time varying form.

The forth of Maxwell's equations is also the same as its non time varying form and is presented in equation (3.14)

$$
\nabla \cdot \vec{B}=0
$$

and its integral form is presented in equation (3.15)

$$
\int_{S} \vec{B} \cdot d \vec{S}=0
$$

The equations that are presented are the macroscopic Maxwell equations, which mean that these equations apply to fields in bulk materials such as gases, liquids and solids. Maxwell's equations are valid for time varying fields.

To conclude, the four Maxwell's equations are [1]:

1. Equation $\nabla \times \vec{E}=-\frac{\partial \vec{B}}{\partial t}$, which states that a time-varying magnetic field can generate electric fields.

2. Equation $\nabla \times \vec{H}=\vec{J}+\frac{\partial \vec{D}}{\partial t}$, which states the relationship between a magnetic field and a given current. In the relationship the extra term which is called displacement current is included.

3. Equation $\int_{S} \vec{D} \cdot d \vec{S}=\int_{V} \rho \cdot \mathrm{dV}$, which can be written as $\nabla \cdot \vec{D}=\rho$. This equation states that electric fields can start and end on charges.

4. Equation $\nabla \cdot \vec{B}=0$, which states that there are sources or sinks with magnetic field. This means that $\boldsymbol{B}$ lines never start or end, instead they form closed loops.

Here, it would also be useful to define the magnetic vector potential $\vec{A}$, which is presented in equation (3.16) 
Galvanically Isolated, Wide-Band Current Sensors

$$
\vec{B}=\nabla \times \vec{A}
$$

The magnetic potential can be useful as a computational tool for finding the magnetic field, much in the same way as the electric potential is useful $(\vec{E}=-\nabla V)$.

Like all set's of differential equations, Maxwell's equations cannot be uniquely solved without a suitable set of boundary conditions and initial conditions.

\subsubsection{Boundary Conditions}

It often happens that magnetic or electric fields are present at the boundary of two different materials. This raises the question of how the fields on one side of the boundary are related to those on the other side, and also raises the need of derivation of boundary conditions. The four Maxwell's integral equations can be used to find the boundary conditions of $B, D, H$, and $\boldsymbol{E}$. Boundary conditions are necessary in order to solve Maxwell's equations in partial differential form.

First, let's think of magnetic fields that are present at the boundary of two different materials. Two cases must be considered, the components that are tangential to the boundary and the components that are normal to the boundary. For any materials with different conductivity, equation (3.17) is valid for the tangential components

$$
H_{T 1}=H_{T 2}
$$

and equation (3.18) is valid for normal components

$$
\mu_{1} \cdot \mathrm{H}_{\mathrm{N} 1}=\mu_{2} \cdot \mathrm{H}_{\mathrm{N} 2}
$$

where $\mu$ stands for the permeability of each material.

The equation that is valid for the tangential components of the magnetic field is presented in (3.19)

$$
B_{N 1}=B_{N 2}
$$

and the equation for normal components is presented in (3.20), respectively

$$
D_{N 1}-D_{N 2}=\sigma
$$

where $\sigma$ is the surface charge per unit area on the interface.

At an interface between two dielectrics, equation (3.21) is valid for tangential components

$$
E_{T 1}=E_{T 2}
$$




\subsection{Eddy Currents}

\subsubsection{Main Principle}

The main principle of the generation of eddy currents is presented in Figure 3.1.
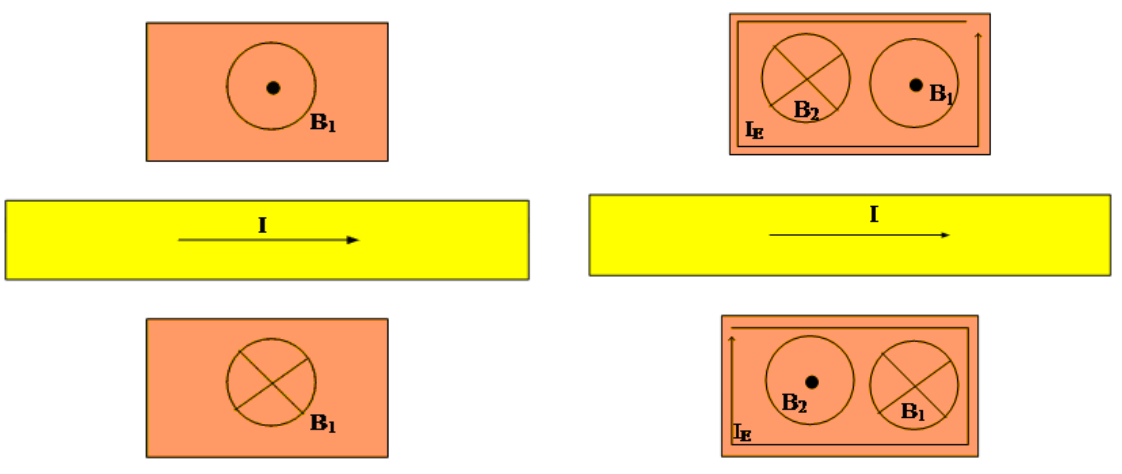

Figure 3.1: Hall plates placed above and below current carrying conductor

As it is presented in Figure 3.1, Hall plates are placed above and below a conductor. Current $I$ is flowing along the conductor. On the left figure, the current carrying conductor induces magnetic field $\boldsymbol{B}$ at the Hall plates in the direction that it is presented in the figure. On the right figure, the eddy currents effect is presented. The primary magnetic field that is induced, induces eddy current in the plates. These eddy currents induce a secondary magnetic field. The secondary magnetic field is opposing to the primary magnetic field.

\subsubsection{Eddy Currents in Hall Plates}

If a conductive device is exposed to a varying magnetic field, eddy currents will be induced to the device [2]. Hall plates are affected by eddy currents. Eddy currents have as a result two effects, which are

- Phase shift and diminution of the magnetic field which acts on the galvanomagnetic device

- Produce an additional Hall effect

According to Lenz's law, eddy currents tend to oppose the charge which induces them. The result of this fact leads to the first effect that is presented above. Suppose that a think, long, rectangular Hall plate is exposed to a perpendicular ac magnetic field. Eddy currents are produced and the density of these eddy currents increases linearly as we approach the plate periphery. The magnetic field which is produced by eddy currents has a maximum along the symmetry axis of the plate [1]. The ratio of this maximum magnetic field and the excitation magnetic field is calculated in equation (3.22) 
Galvanically Isolated, Wide-Band Current Sensors

$$
\left|\frac{B_{m}}{B}\right| \approx \mu_{0} \cdot f \cdot \frac{W}{R_{S}}
$$

where $\mu_{0}$ is the carrier mobility, $\mathrm{f}$ is the frequency of the excitation magnetic field, $R_{S}$ is the sheet resistance of the plate and $W$ is the width of the plate. The sheet resistance can be calculated by equation (3.23)

$$
R_{S}=\frac{\rho}{\mathrm{d}}=\frac{1}{\sigma \cdot \mathrm{d}}
$$

where $d$ is the thickness of the plate, $\rho$ is the resistivity of the plate and $\sigma$ is the conductivity of the plate.

The second effect caused by the eddy currents is the production of an additional Hall effect. For an ac excitation magnetic field, the total current density in the plate is given by the summation of the current density in the Hall plate produced by the input current $\left(J_{i}\right)$ and the eddy current density $\left(J_{e}\right)$ as it is described in equation (3.24)

$$
J_{\text {total }}=J_{i}+J_{e}
$$

The total magnetic field acting on the plate is presented in equation (3.25)

$$
B \approx B_{i}+B_{S}
$$

where $B_{i}$ is the input magnetic field and $B_{S}$ is the self-field produced in the plate by the excitation current. The magnetic field produced by eddy currents is neglected in equation (3.24). Thus, the Hall voltage can be obtained by equation (3.26)

$$
V_{H} \approx V_{H i}+V_{H e}
$$

In equation (3.24), is not used the symbol of exact equality, because the calculation of $B_{S}$ is approximated by a linear function. Here, $V_{H i}$ is the ordinary Hall voltage and $V_{H e}$ is the Hall voltage produced by the coupling of the self magnetic field and eddy currents. The ratio of these two voltages is calculated in equation (3.27)

$$
\left|\frac{V_{H e}}{V_{H i}}\right|=\mu_{0} \cdot \mathrm{f} \cdot \frac{\mathrm{W}}{\mathrm{R}_{\mathrm{S}}}
$$

This ratio is quite the same as the one described in equation (3.22). This means that these two effects become noticeable for about the same frequency.

\subsubsection{Skin Effect}

Conductors may be used to transmit electrical energy or signals, using an ac current flowing through that conductor. The charge carriers constituting that current are accelerated by an electric field. An alternating current may also be induced in a conductor due to an alternating magnetic field, as it is already described above. The current density is found to be greater at the conductor's surface. 
Galvanically Isolated, Wide-Band Current Sensors

The skin effect in semiconductors and Hall plates consecutively, is due to opposing eddy currents induced by the changing magnetic field resulting from the ac current. Skin effect is the tendency of an alternating electric current (ac) to distribute itself within a conductor with the current density being largest near the surface of the conductor and decreasing at greater depths. In other words, the electric current flows mainly at the "skin" of the conductor, at an average depth called the skin depth. The skin effect causes the effective resistance of the conductor to increase at higher frequencies where the skin depth is smaller. Usually, at high frequencies the average depth at which the electric current is flowing is much smaller.

Skin depth depends on the conductivity and permeability of the conductor. Skin depth is inversely proportional to the square root of the permeability of the conductor. 
Galvanically Isolated, Wide-Band Current Sensors

\section{References}

[1] William H. Hayt, John A. Buck, Engineering Electromagnetics, 2000

D.K. Cheng, Field and Wave Electromagnetics, Wesley 1983

http://www.scribd.com/doc/24672554/David-K-Cheng-Field-and-Wave-

Electromagnetics

[2] R.S. Popovic, Hall Effect Devices, IOP Publishing, 2004 


\section{Readout Circuit}

\subsection{Basic Circuits and Building Blocks}

\subsubsection{The CMOS Differential Cell (Basic Transconductance)}

Figure 4.1 shows the basic CMOS differential pair. It consists of a pair of NMOS transistors (MNO and MN1) and a pair of PMOS transistors (MPO and MP1), biased with a constant current source $l$. This source in the actual implementation of the circuit is implemented using the bias cell. The resistance which is in parallel to the current source and is equal to $\left(1 / \lambda \cdot \mathrm{I}_{\mathrm{BIAS}}\right)$ stands for the channel-length modulation effect.

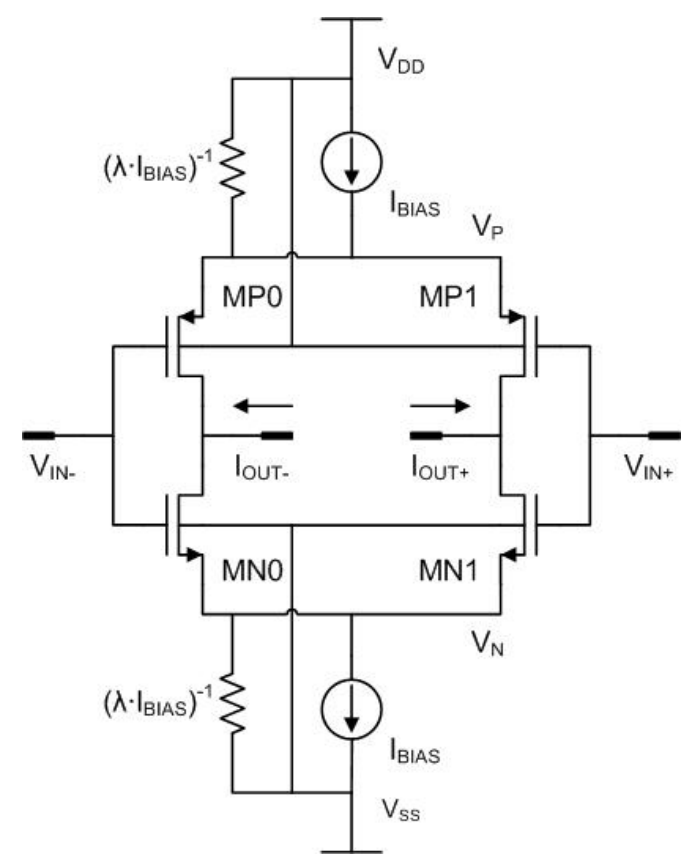

Figure 4.1: The CMOS differential pair

The circuit of Figure 4.1 has two differential voltage inputs and two differential current outputs, thus it makes a differential transconductance amplifier. The circuit of Figure 4.1 can be described by the following equations (4.1)-(4.4).

$$
\begin{aligned}
& v_{I N+}=v_{C M}+\frac{\Delta v}{2} \\
& v_{I N-}=v_{C M}-\frac{\Delta v}{2}
\end{aligned}
$$

$$
\begin{aligned}
& i_{\text {OUT+ }}=i_{C M}+\frac{\Delta i}{2} \\
& i_{\text {OUT- }}=i_{C M}-\frac{\Delta i}{2}
\end{aligned}
$$

In the general case of $\lambda \neq 0$, the following equations can be calculated.

$$
\mathrm{i}_{\text {BIASN }}=i_{B I A S N 0}+i_{B I A S N 1}
$$


Galvanically Isolated, Wide-Band Current Sensors

$$
\begin{aligned}
& i_{B I A S N 0}=\frac{1}{2} K_{n} \frac{W_{n}}{L_{n}}\left(\left(v_{I N-}-v_{N}\right)-V_{t N}\right)^{2} \\
& i_{B I A S N 1}=\frac{1}{2} K_{n} \frac{W_{n}}{L_{n}}\left(\left(v_{I N+}-v_{N}\right)-V_{t N}\right)^{2}
\end{aligned}
$$

After calculations and substitution of equations (4.1)-(4.4), the following equation is derived

$$
\begin{aligned}
& \frac{i_{B I A S N}}{(1 / 2) K_{n}\left(W_{n} / L_{n}\right)}=2\left[\left(v_{C M}^{2}+v_{N}^{2}+V_{t N}^{2}-2 v_{N} v_{C M}-2 v_{C M} V_{t N}+2 v_{N} V_{t N}\right)+\frac{\Delta \mathrm{v}^{2}}{4}\right] \Rightarrow \\
& \frac{i_{B I A S N}}{(1 / 2) K_{n}\left(W_{n} / L_{n}\right)}=2\left[\left(v_{C M}-v_{N}-V_{t N}\right)^{2}+\frac{\Delta \mathrm{v}^{2}}{4}\right] \\
& \Rightarrow
\end{aligned}
$$

$i_{B I A S N}=\beta_{\mathrm{n}}\left[\left(v_{C M}-v_{N}-V_{t N}\right)^{2}+\frac{\Delta \mathrm{v}^{2}}{4}\right]$

Where $\beta_{\mathrm{n}}=K_{n} \frac{W_{n}}{L_{n}} \quad, \quad K_{n}=\mu_{\mathrm{n}} \cdot C_{\mathrm{ox}}$

Following the same calculation procedure the corresponding $i_{B I A S P}$ current can be calculated in equation (4.6).

$i_{\text {BIASP }}=\beta_{\mathrm{p}}\left[\left(v_{C M}-v_{P}-V_{t P}\right)^{2}+\frac{\Delta \mathrm{v}^{2}}{4}\right]$

Where $\beta_{\mathrm{p}}=K_{p} \frac{W_{p}}{L_{p}} \quad, \quad K_{p}=\mu_{\mathrm{p}} \cdot C_{o x}$

Symbols $i_{B I A S N}$ and $i_{B I A S P}$ stand for the current connected to the NMOS and PMOS pairs respectively.

In the following equations (4.7) and (4.8), currents $\Delta \mathrm{i}_{\mathrm{N}}$ and $\Delta \mathrm{i}_{\mathrm{P}}$ are calculated. These currents stand for the differential currents of NMOS and PMOS pairs respectively.

$$
\begin{aligned}
& \Delta \mathrm{i}_{\mathrm{N}}=\beta_{\mathrm{N}} \cdot \Delta v\left(v_{C M}-v_{N}-V_{t N}\right) \\
& \Delta \mathrm{i}_{\mathrm{P}}=-\beta_{\mathrm{P}} \cdot \Delta \mathrm{v}\left(\mathrm{v}_{\mathrm{CM}}-v_{P}-V_{t P}\right)
\end{aligned}
$$

Solving equations (4.5) and (4.6) for $v_{N}$ and $v_{P}$, equations (4.9) and (4.10) can be easily derived.

$v_{N}=v_{C M}-V_{t N}-\sqrt{\frac{i_{B L A S N}}{\beta_{\mathrm{n}}}-\frac{\Delta \mathrm{v}^{2}}{4}}$ 
Galvanically Isolated, Wide-Band Current Sensors

$$
\begin{aligned}
& v_{P}=v_{C M}-V_{t P}+\sqrt{\frac{i_{B I A S P}}{\beta_{\mathrm{P}}}-\frac{\Delta \mathrm{v}^{2}}{4}} \\
& i_{B I A S N}=I_{B I A S}+\lambda \cdot \mathrm{I}_{\mathrm{BIAS}} \cdot v_{N} \\
& i_{B I A S P}=I_{B I A S}-\lambda \cdot \mathrm{I}_{\mathrm{BIAS}} \cdot v_{P}
\end{aligned}
$$

Assuming that the NMOS and PMOS transistors are matched, this means that $\beta_{\mathrm{n}}=\beta_{\mathrm{p}}=\beta$ and $\mathrm{V}_{\mathrm{t}}=V_{t N}=-V_{t P}$ equations (4.13)-(4.16) can be derived. These equations describe the performance of the basic transconductance.

$$
\begin{aligned}
& \Delta \mathrm{i}=\Delta \mathrm{i}_{\mathrm{N}}+\Delta \mathrm{i}_{\mathrm{P}}=\beta \cdot \Delta \mathrm{v}\left(\mathrm{v}_{\mathrm{P}}-v_{n}\right) \approx \Delta \mathrm{v} \sqrt{2 \cdot \beta \cdot \mathrm{I}_{\mathrm{BIAS}}-\beta^{2} \cdot \Delta \mathrm{v}^{2}} \\
& i_{C M}=\frac{i_{B I A S N}-i_{B I A S P}}{2}=\frac{\lambda \cdot \mathrm{I}_{\mathrm{BIAS}}}{2} \cdot\left(v_{N}+v_{P}\right) \approx \lambda \cdot \mathrm{I}_{\mathrm{BIAS}} \cdot v_{C M} \\
& \Delta \mathrm{i} \approx \mathrm{G}_{\mathrm{D}} \cdot \Delta \mathrm{v} \text { where } G_{D}=\sqrt{2 \cdot \beta \cdot \mathrm{I}_{\mathrm{BIAS}}-\beta^{2} \cdot \Delta \mathrm{v}} \\
& i_{C M} \approx G_{C M} \cdot v_{C M} \quad \text { where } G_{C M}=\lambda \cdot \mathrm{I}_{\mathrm{BIAS}}
\end{aligned}
$$




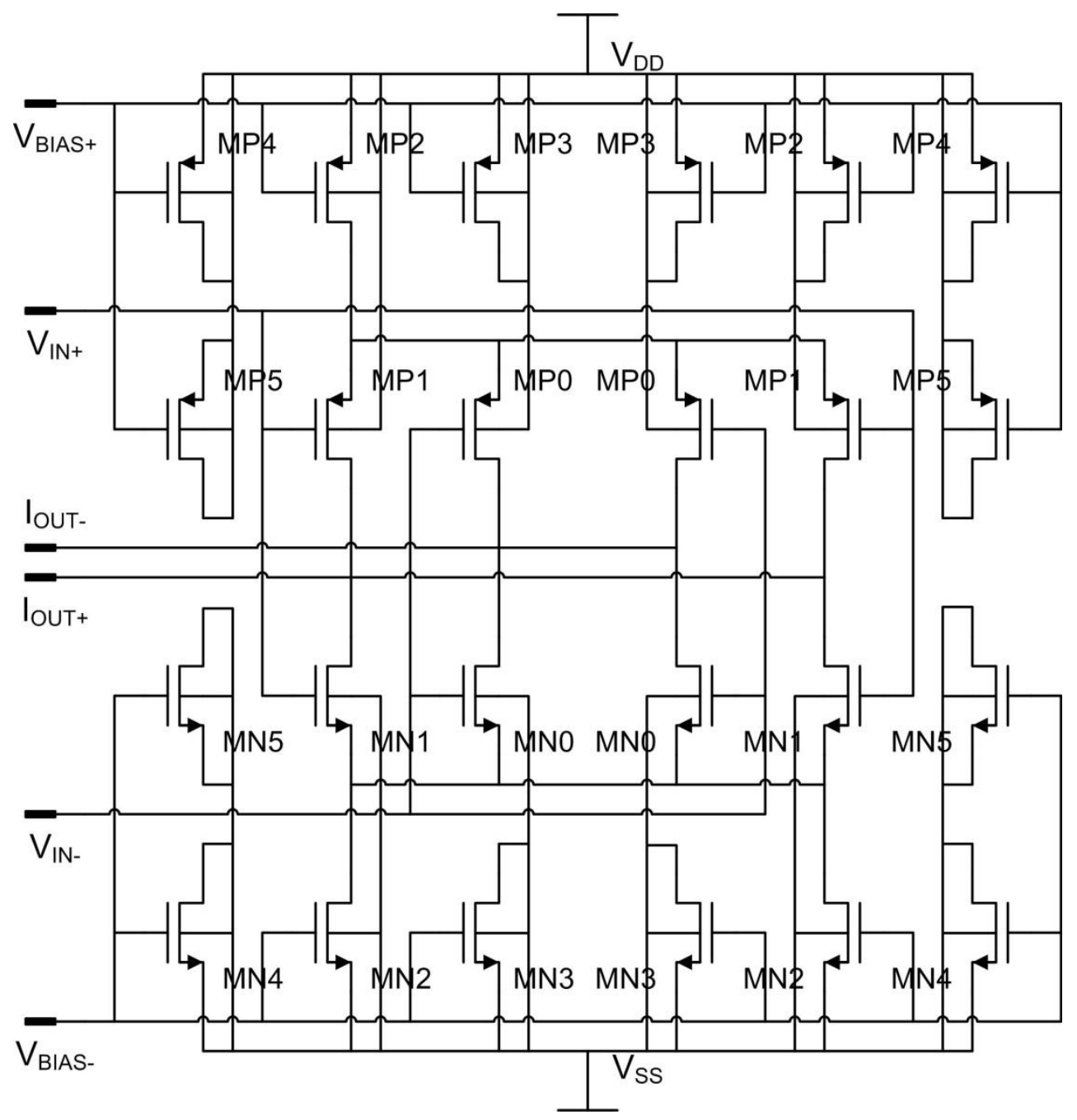

Figure 4.2: Actual implementation of the Basic Transconductance Circuit

In Figure 4.2 the actual implementation of the Transconductance Amplifier is presented. Two pairs of MOS current sources are used, which consist of the transistors MN2, MN3, MP2 and MP3. Also two pairs of MOS capacitors are added, these consist of the transistors MN4, MN5, MP4 and MP5. These capacitors are also used as dummy structures in order to protect transistors MN1, MN2, MP1 and MP2 from process gradients.

The dummy structures are used because of the asymmetry of some parts of the circuit, for example regarding transistors MN1 and MNO. When the layout of this circuit is designed these transistors do not see the same environment. Thus, dummy structures are added, in order to reduce the mismatch and the asymmetry.

The Transconductance Amplifier is connected in specific topologies in the final circuit. These topologies will be analyzed below. 
Galvanically Isolated, Wide-Band Current Sensors

\subsubsection{CMOS Current Mirror}

The CMOS current mirror is presented in Figure 4.3.

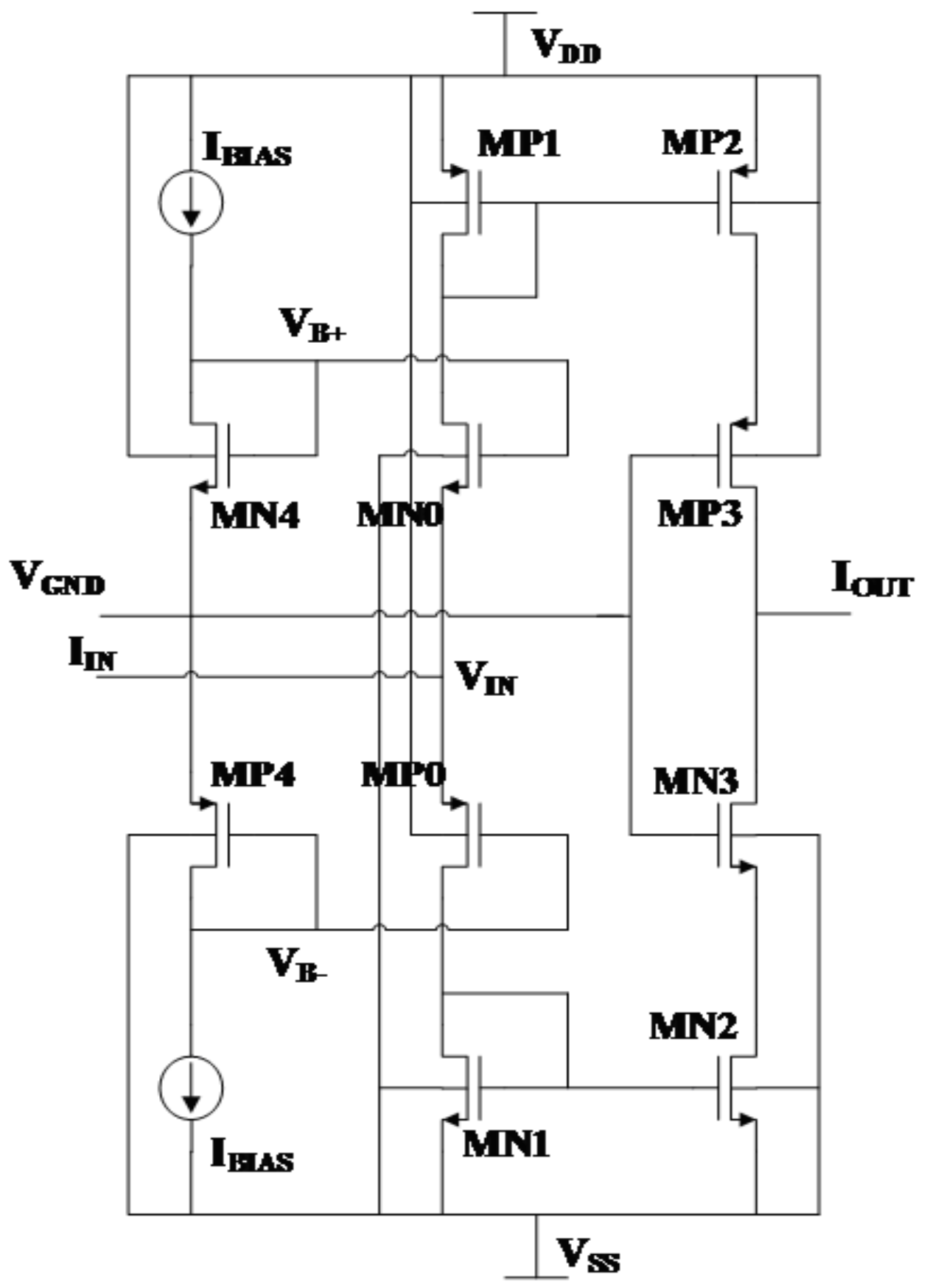

Figure 4.3: CMOS Current Mirror

The input current of the CMOS current mirror is symbolised by $I_{I N}$ and the output current is $I_{\text {OUT }}$. The NMOS and PMOS transistors are matched, this means that $\beta_{\mathrm{n}}=\beta_{\mathrm{p}}=\beta=K(W / L)$ and $\mathrm{V}_{\mathrm{t}}=V_{t N}=-V_{t P}$. Ideally, no current enters GND terminal, which is connected to the Gates of MN3 and MP3 transistors. This means that the input resistance is infinite. 
Galvanically Isolated, Wide-Band Current Sensors

The calculation of $V_{B+}$ and $V_{B-}$ can be easily derived in (4.17) and (4.18).

$$
\begin{aligned}
& I_{B I A S}=\frac{1}{2} \cdot \beta \cdot\left(V_{B+}-V_{G N D}-V_{t}\right)^{2} \\
& \mathrm{~V}_{\mathrm{B}+}=V_{G N D}+V_{t}+\sqrt{\frac{2 \cdot I_{B I A S}}{\beta}} \\
& \mathrm{V}_{\mathrm{B}-}=V_{G N D}-V_{t}-\sqrt{\frac{2 \cdot I_{B I A S}}{\beta}}
\end{aligned}
$$

Currents $i_{M N 0}$ and $i_{M P 0}$ that are flowing through transistors MNO and MPO respectively, are calculated below in (4.19) and (4.20).

$$
\begin{aligned}
& i_{M N 0}=\frac{1}{2} \beta\left(\mathrm{V}_{\mathrm{B}+}-v_{I N}-V_{t}\right)^{2}=\frac{1}{2} \beta\left(\mathrm{V}_{\mathrm{GND}}+\sqrt{\frac{2 I_{B I A S}}{\beta}-\mathrm{V}_{\mathrm{IN}}}\right)^{2} \\
& i_{M N 0}=\frac{1}{2} \beta\left(\mathrm{V}_{\mathrm{GND}}-v_{I N}\right)^{2}+\sqrt{2 \cdot \beta \cdot \mathrm{I}_{\mathrm{BIAS}}}\left(V_{G N D}-v_{I N}\right)+I_{B I A S} \\
& i_{M P 0}=\frac{1}{2} \beta\left(\mathrm{V}_{\mathrm{B}-}-v_{I N}+V_{t}\right)^{2}=\frac{1}{2} \beta\left(\mathrm{V}_{\mathrm{GND}}-\sqrt{\frac{2 I_{B I A S}}{\beta}-v_{I N}}\right)^{2} \\
& i_{M P 0}=\frac{1}{2} \beta\left(\mathrm{V}_{\mathrm{GND}}-v_{I N}\right)^{2}-\sqrt{2 \cdot \beta \cdot \mathrm{I}_{\mathrm{BIAS}}}\left(V_{G N D}-v_{I N}\right)+I_{B I A S}
\end{aligned}
$$

The input $i_{I N}$ current can be calculated in equation (4.21), by using equations (4.19) and (4.20), as it is presented bellow.

$$
\begin{aligned}
& i_{I N}=i_{M P 0}-i_{M N 0} \\
& i_{I N}=2 \sqrt{2 \cdot \beta \cdot \mathrm{I}_{\mathrm{BIAS}}}\left(v_{I N}-V_{G N D}\right)=\frac{v_{I N}-V_{G N D}}{Z_{I N}} \\
& Z_{I N}=\frac{1}{2 \sqrt{2 \cdot \beta \cdot \mathrm{I}_{\mathrm{BIAS}}}} \\
& v_{I N}=i_{I N} \cdot Z_{I N}+V_{G N D}
\end{aligned}
$$

The input current is copied through the current mirror to the output, thus equation (4.24) can easily be derived.

$$
i_{\text {OUT }}=A_{I} \cdot\left(i_{M P 0}-i_{M N 0}\right)=A_{I} \cdot i_{I N}
$$

The actual implementation of the CMOS current mirror is presented in Figure 4.4. 
Galvanically Isolated, Wide-Band Current Sensors

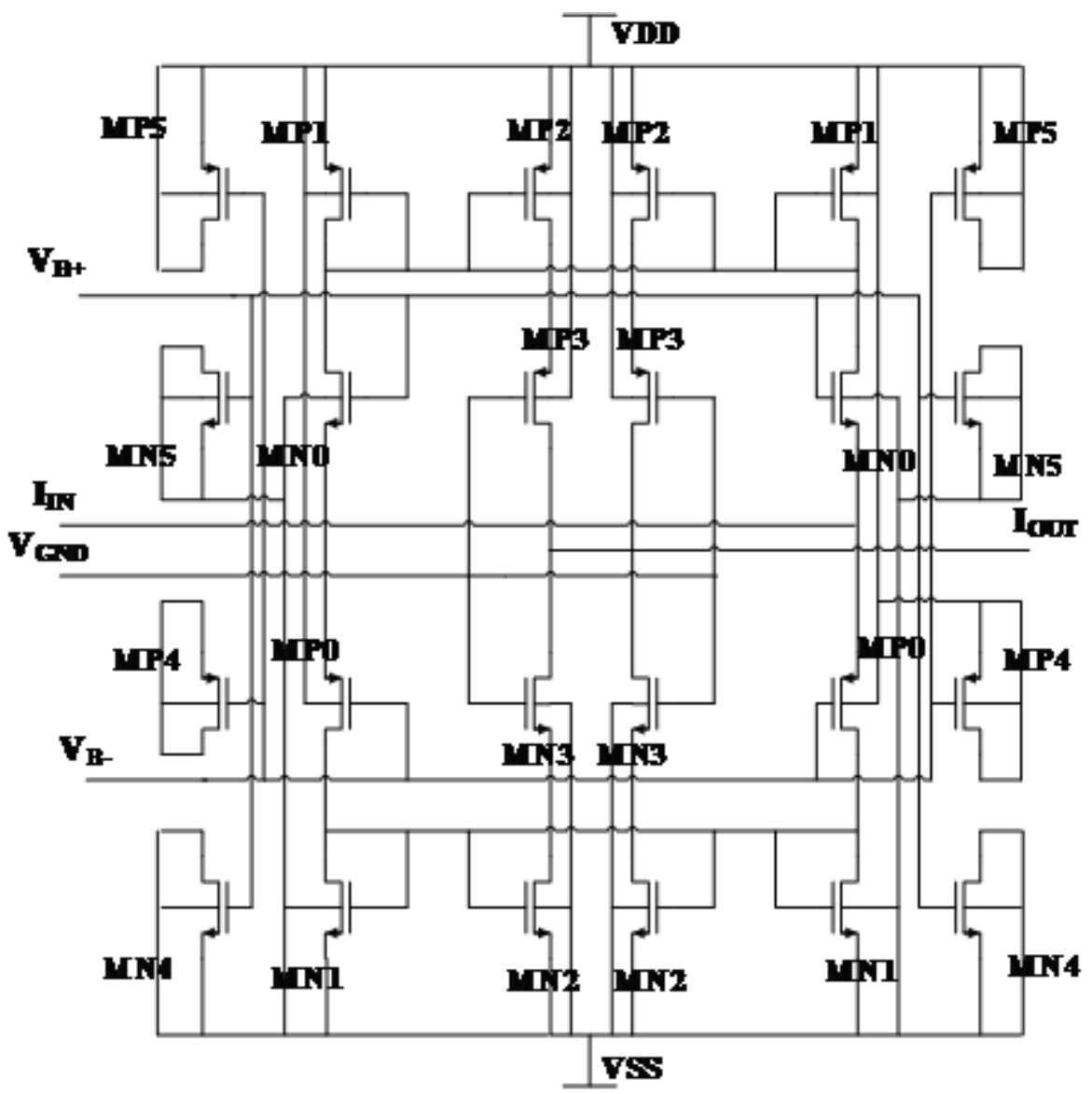

Figure 4.4: Actual implementation of the CMOS Current Mirror

Two pairs of MOS capacitors are added (MP4, MN4, MP5 and MN5) and are used as dummy structures in order to protect transistors MN0, MN1, MP0 and MP1 from process gradients. 
Galvanically Isolated, Wide-Band Current Sensors

\subsubsection{Bias Circuit}

The Bias circuit is presented in Figure 4.5.

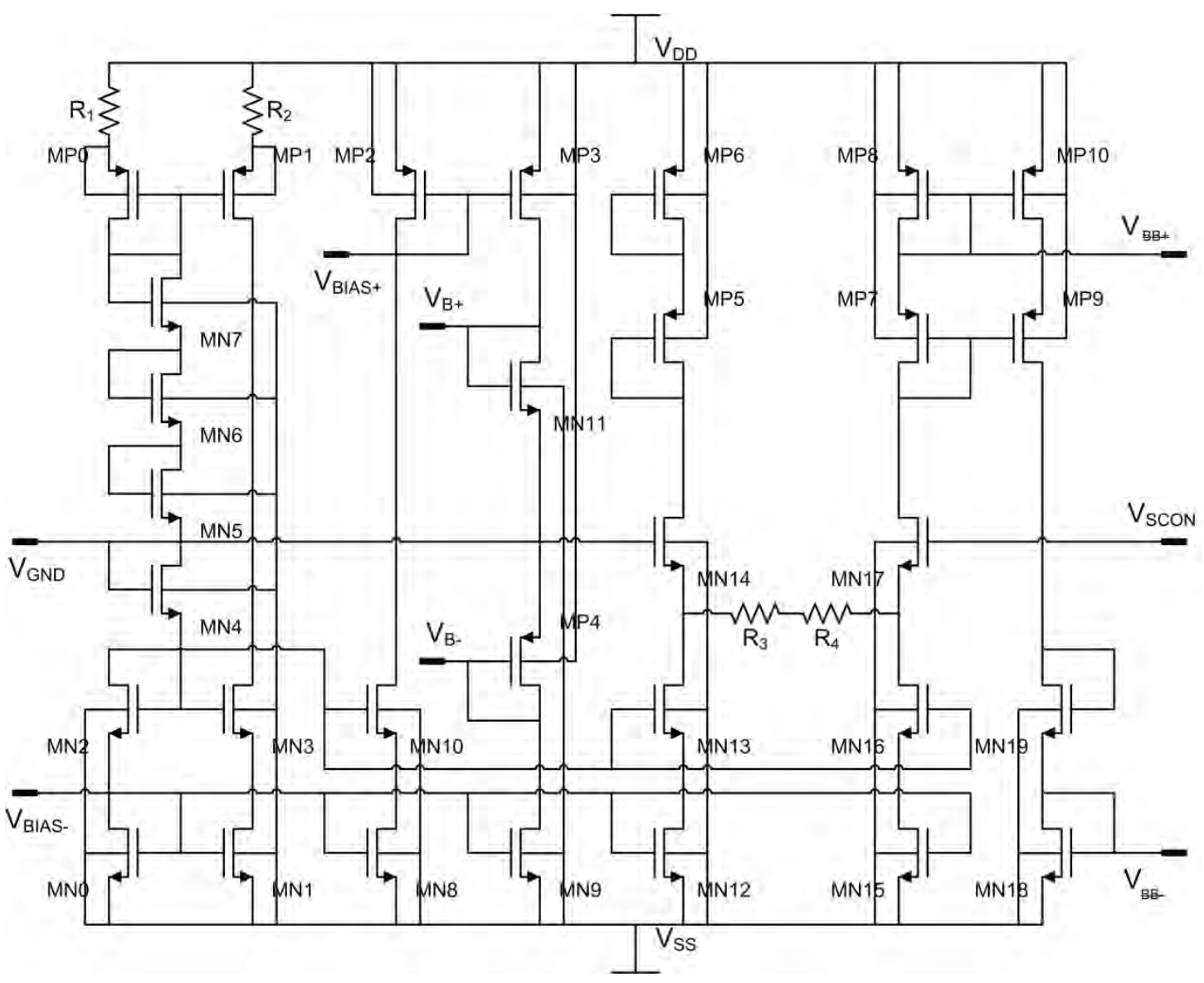

Figure 4.5: Bias Circuit

The bias circuit generates voltages a) $V_{B I A S_{+}}, V_{B I A S_{-}}$, b) $V_{B+}, V_{B-}$ and c) $V_{B B_{+}}, V_{B B_{-}-}$. The values of the later voltages (c) are controlled by the external voltage $V_{S C O N}$ and can be used for gain-amplitude control of the transconductance amplifier as it will be explained later.

The current $I_{B I A S}$ can be calculated in (4.25).

$I_{\text {BIAS }}=\frac{\left(\sqrt{\beta_{1}}-\sqrt{\beta_{0}}\right)^{2}}{\beta \cdot\left(R_{2}-R_{1}\right)^{2}}$

Where $\beta_{1}, \beta_{0}$ and $\beta$ are standing for the beta parameter of transistors MP1, MP0 and MN0MN3 respectively. Regarding the fact that both of the resistors $R_{1}$ and $R_{2}$ exhibit different TC's (temperature coefficient), their values may be adjusted so as to a) set $I_{B I A S}$ and b) stabilize it against temperature.

Transistors MN4-MN7 serve as startup circuit as well as to produce the $V_{G N D}$ signal. Transistors MN8-MN11 and MP2-MP4 generate the biasing voltage signals. Transistors 
Galvanically Isolated, Wide-Band Current Sensors

MN12-MN13 and MN15-MN16 serve as current sources biasing the differential cell made of MN14 and MN17 that has active load built of MP5-MP8. $V_{S C O N}$ signal can be employed to generate a variable biasing current in MN18-MN19 and MP9-MP10. ((Resistors $R_{3}, R_{4}$ are used as negative feedback to stabilize the transconductance of the differential cell. Their TC values are complementary, so as to stabilize the response of the cell against temperature)). The biasing circuit has an extra functionality. The circuit can be used and generate biasing for both cases of differential or not differential input. In other words it can bias circuits with the options of $V_{C M}=0 \mathrm{~V}$ or $V_{C M}=1.6 \mathrm{~V}$. At this point, it should be noticed that the supply voltage for the first case is equal to $V_{D D}=3.2 \mathrm{~V}$ and the ground voltage is equal to $V_{S S}=0 \mathrm{~V}$. When $V_{C M}=1.6 \mathrm{~V}$ is used, the supply voltage is equal to $V_{D D}=+1.6 \mathrm{~V}$ and the ground voltage is equal to $V_{S S}=-1.6 \mathrm{~V}$. This functionality can be applied because of the fact that the biasing circuit also generates $V_{G N D}$ voltage. This option was necessary at the beginning of the design, since there was a doubt regarding the $V_{C M}$ voltage of the inputs of the complete circuit that is described here.

\subsubsection{Operational Transconductance Amplifier}

Figure 4.6 presents the configuration of the Operational Transconductance Amplifier, hereafter called OTA. The Operational Transconductance Amplifier consists of two Basic Transconductance Amplifiers connected in feedback configuration, as it is presented in Figure 4.6.

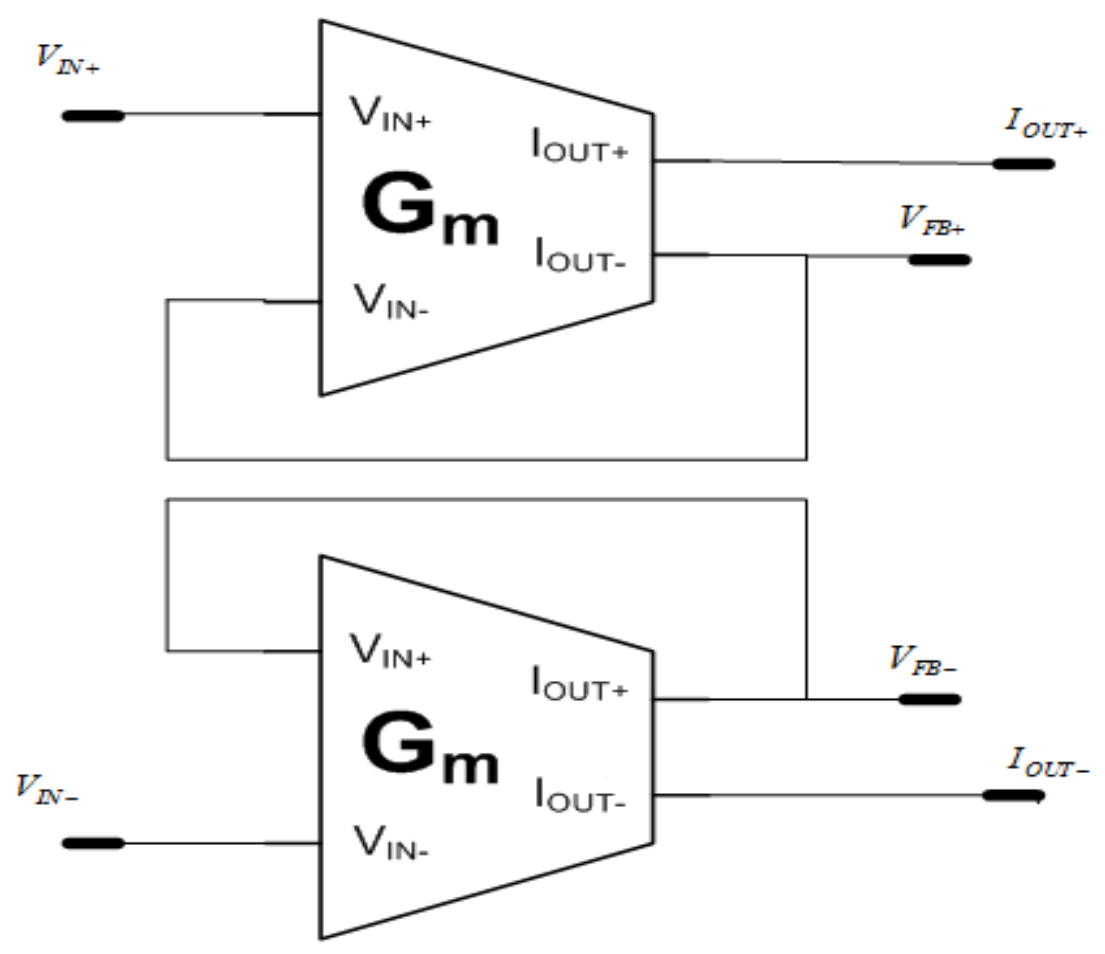

Figure 4.6: Operational Transconductance Amplifier (OTA) 
Galvanically Isolated, Wide-Band Current Sensors

The Transconductance Amplifier is connected in two different configurations in the final system design. The first configuration is presented in Figure 4.7. In this configuration the outputs $V_{F B+}$ and $V_{F B-}$ are connected to capacitors. The two capacitors that are connected to these pins have equal values. From inspection of Figure 4.7, the voltages $V_{F B+}, V_{F B-}$ can be calculated in equations (4.26) and (4.27).

$$
\begin{aligned}
& V_{F B+}(\omega)=\frac{1}{j \cdot \omega \cdot \operatorname{cap} 1}\left(-I_{\text {out }}(\omega)\right) \\
& \mathrm{V}_{\mathrm{FB}-}(\omega)=\frac{1}{\mathrm{j} \cdot \omega \cdot \operatorname{cap} 2}\left(-I_{\text {out }}(\omega)\right)
\end{aligned}
$$

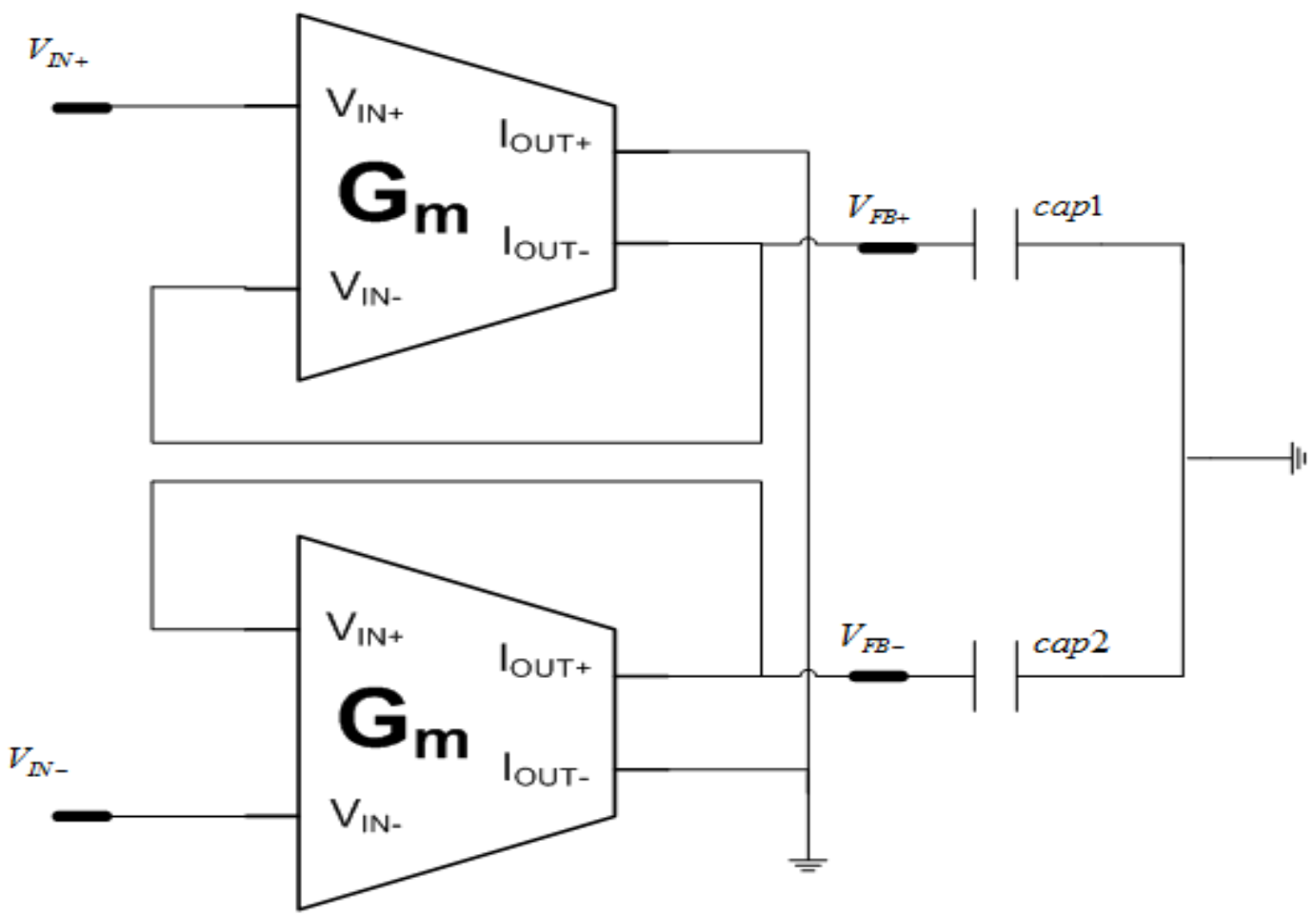

Figure 4.7: First OTA Configuration

Also, equations (4.28) and (4.29) are valid for the circuit of Figure 4.7.

$$
\begin{aligned}
& V_{F B+}=A_{G} \cdot V_{I N+} \\
& V_{F B-}=A_{G} \cdot V_{I N-}
\end{aligned}
$$

Where $A_{G}$ is the amplifier's gain.

From now on the OTA with feedback will be represented by the symbol presented in Figure 4.8 . 
Galvanically Isolated, Wide-Band Current Sensors

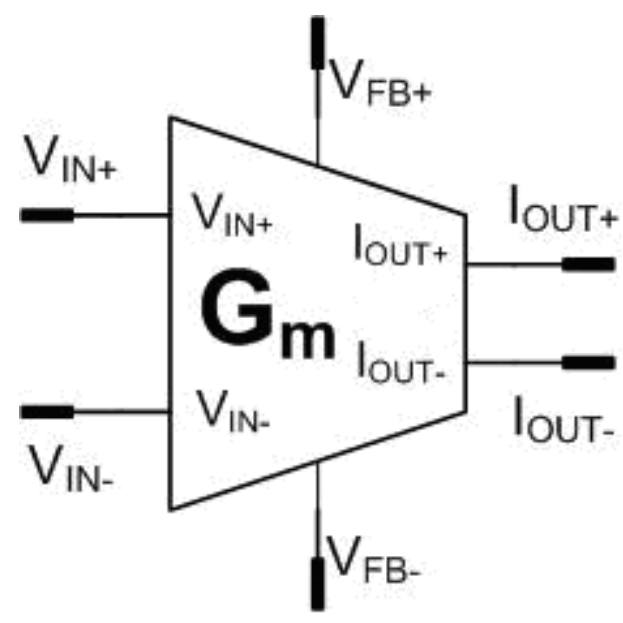

Figure 4.8: OTA Symbol

The second configuration of the OTA is presented in Figure 4.9. This second circuit consists of Basic Transconductance Amplifiers, Current Mirrors and Resistors. It consists of two feedback resistors $R_{F 1}=R_{F 2}=R_{F}$ and two output resistors $R_{O 1}=R_{O 2}=R_{O}$.

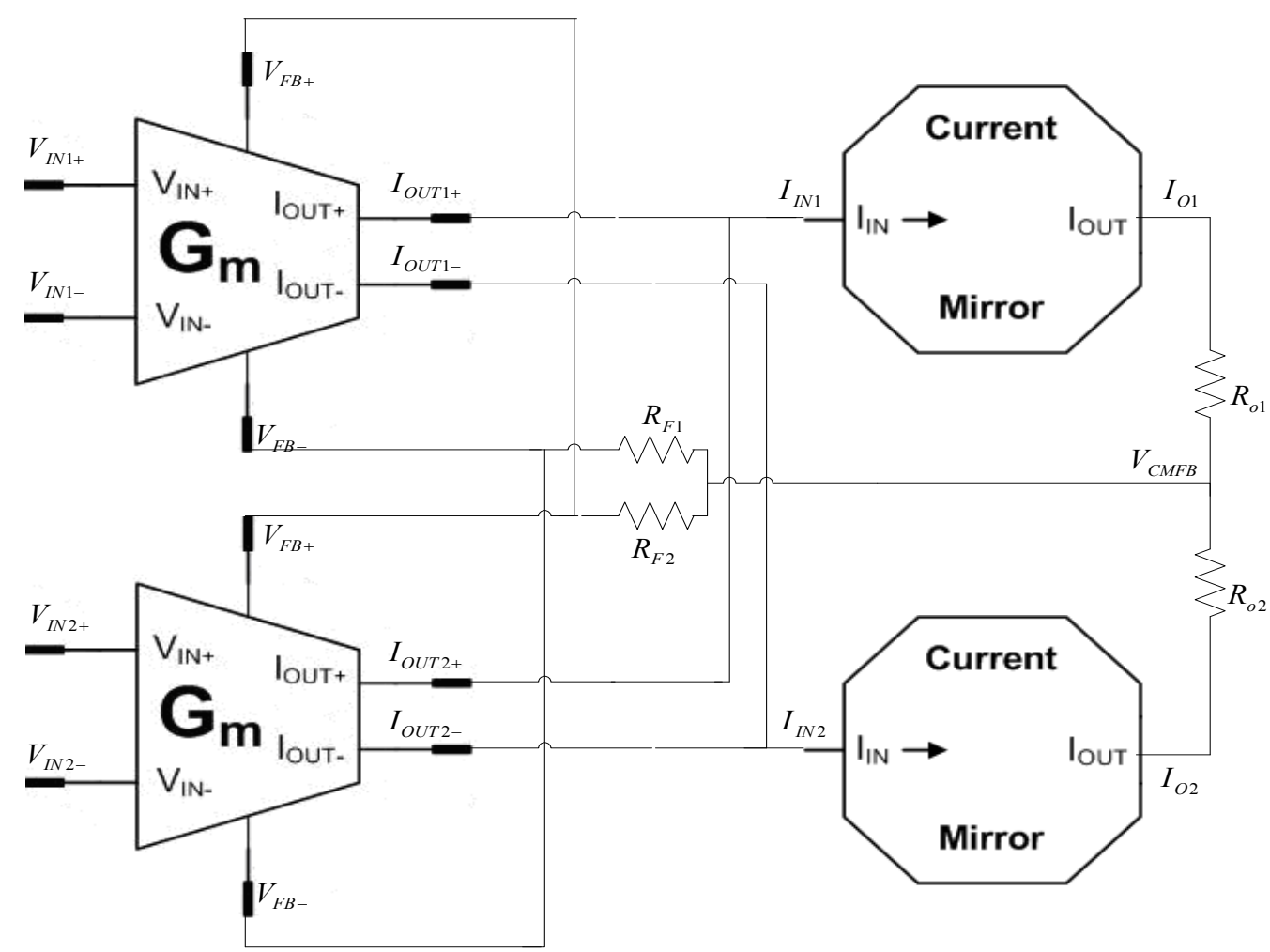

Figure 4.9: Second OTA Configuration

The output currents $I_{O 1}$ and $I_{O 2}$ can be calculated using equations (4.1)-(4.4), (4.15), (4.16) and (4.24), as follows.

$i_{O 1}=A_{I} \cdot i_{I N 1}$ 
Galvanically Isolated, Wide-Band Current Sensors

$$
\begin{aligned}
& i_{O 2}=A_{I} \cdot i_{I N 2} \\
& i_{I N 1}=\left(i_{O U T 1+}+i_{\text {OUT } 2+}\right) \\
& i_{I N 1}=\left[G_{C M}\left(\frac{v_{I N 1+}+v_{F B+}}{2}\right)+G_{D}\left(\frac{v_{I N 1+}-v_{F B+}}{2}\right)+G_{C M}\left(\frac{v_{I N 2+}+v_{F B+}}{2}\right)+G_{D}\left(\frac{v_{I N 2+}-v_{F B+}}{2}\right)\right] \\
& i_{I N 1}=\left[G_{C M}\left(\frac{v_{I N 1+}+v_{I N 2+}+2 \cdot v_{F B+}}{2}\right)+G_{D}\left(\frac{v_{I N 1+}+v_{I N 2+}-2 \cdot v_{F B+}}{2}\right)\right] \\
& i_{I N 2}=\left(i_{O U T 1-}+i_{O U T 2-}\right) \\
& i_{I N 2}=\left[G_{C M}\left(\frac{v_{I N 1-}+v_{F B-}}{2}\right)+G_{D}\left(\frac{v_{I N 1-}-v_{F B-}}{2}\right)+G_{C M}\left(\frac{v_{I N 2-}+v_{F B-}}{2}\right)+G_{D}\left(\frac{v_{I N 2-}-v_{F B-}}{2}\right)\right] \\
& i_{I N 2}=\left[G_{C M}\left(\frac{v_{I N 1-}+v_{I N 2-}+2 \cdot v_{F B-}}{2}\right)+G_{D}\left(\frac{v_{I N 1-}+v_{I N 2-}-2 \cdot v_{F B-}}{2}\right)\right] \\
& v_{C M F B}=Z_{C M F B}\left(A_{I}+1\right)\left(i_{I N 1}+i_{I N 2}\right)
\end{aligned}
$$

Where $Z_{C M F B}$ is the total impedance of the node $v_{C M F B}$ against the analog ground.

$$
\begin{aligned}
& v_{F B-}=v_{C M F B}+R_{F} \cdot i_{I N 2} \\
& v_{F B+}=v_{C M F B}+R_{F} \cdot i_{I N 1}
\end{aligned}
$$

Substituting equations (4.34) and (4.33) in equations (4.30) and (4.31) respectively, the following equations (4.35) and (4.36) can be derived.

$$
\begin{aligned}
& i_{I N 1}=\frac{G_{C M}+G_{D}}{1-G_{C M} \cdot R_{F}+G_{D} \cdot R_{F}}\left(\frac{v_{I N 1+}+v_{I N 2+}}{2}\right)-\frac{G_{D}-G_{C M}}{1-G_{C M} \cdot R_{F}+G_{D} \cdot R_{F}} v_{C M F B} \\
& i_{I N 2}=\frac{G_{C M}+G_{D}}{1-G_{C M} \cdot R_{F}+G_{D} \cdot R_{F}}\left(\frac{v_{I N 1-}+v_{I N 2-}}{2}\right)-\frac{G_{D}-G_{C M}}{1-G_{C M} \cdot R_{F}+G_{D} \cdot R_{F}} v_{C M F B}
\end{aligned}
$$




\subsection{System Implementation}

This circuit is designed for use as a readout circuit for current sensing applications. The circuit has wide bandwidth that ranges from $\mathrm{DC}$ up to $1 \mathrm{MHz}$. In order to achieve this functionality two types of magnetic sensors are used, one capturing low frequency components and one for high frequencies. A Hall device serves the first purpose and a pick-up coil printed on a PCB board serves the second. Due to offset voltage and temperature stability, Hall sensors are limited to measurements up to few $\mathrm{KHz}$. Pick-up coil performance is not good at $\mathrm{DC}$, but is performing well at higher frequencies.

This work includes two tape-outs and accordingly there were two system designs. The first circuit design is presented in Figure 4.10.

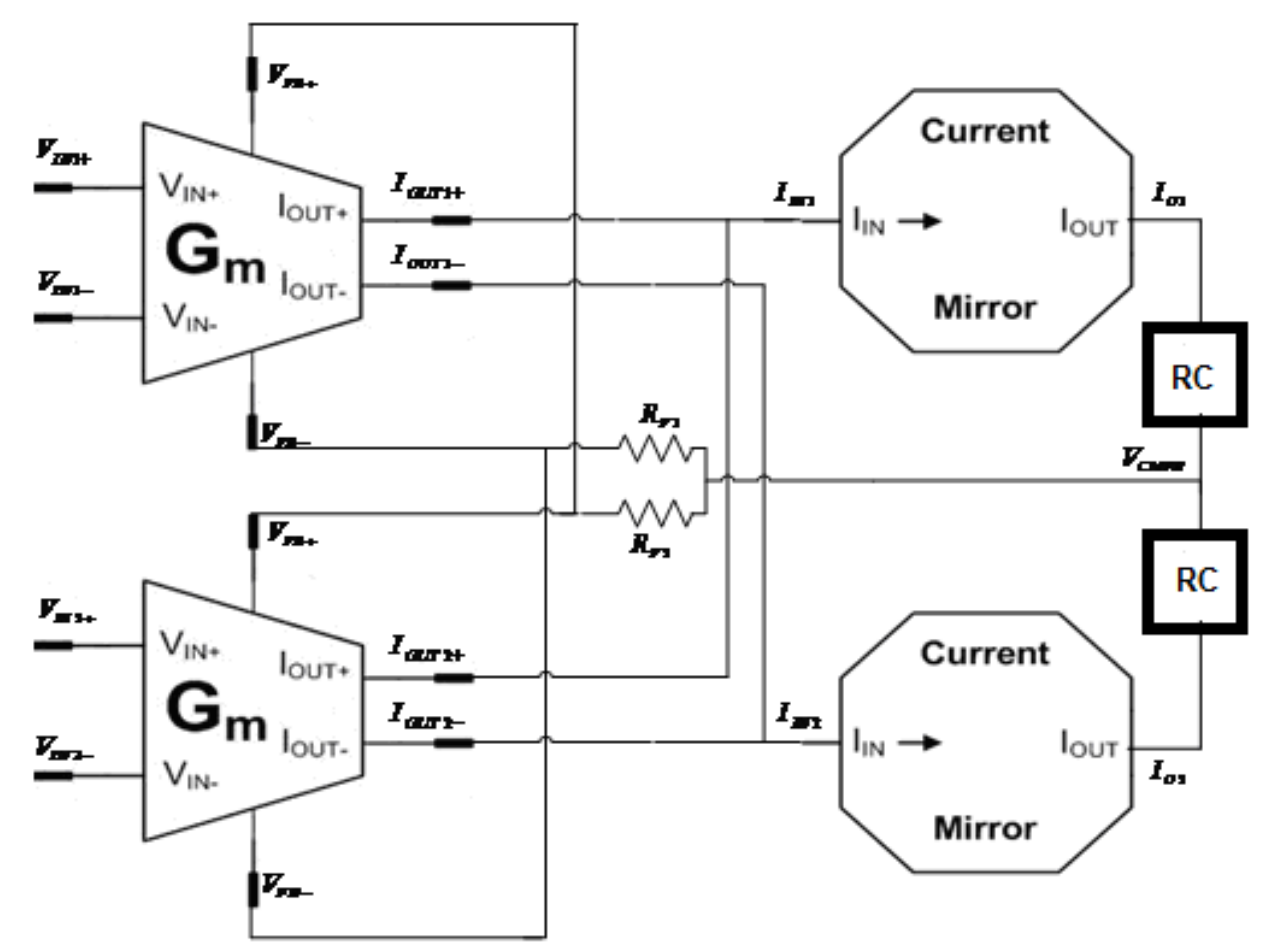

Figure 4.10: First design of the Readout Circuit

The Final Readout Circuit that is designed is presented in Figure 4.11. This readout circuit, merges the signal from the coil and the signal from a Hall sensor.

The difference between the two designs is that the first design does not have an amplifier with output capacitors in order to exact match the frequency response of the output signal. The two designs are quite similar, so the following description of the second design also corresponds to the first one. 
To the writer's knowledge, the only counterpart attempt that was done in the past is described in [1]. The difference with that design concept is that the second input is a Rogowski coil instead of a pick-up coil that is used here. The first input is also a Hall device.

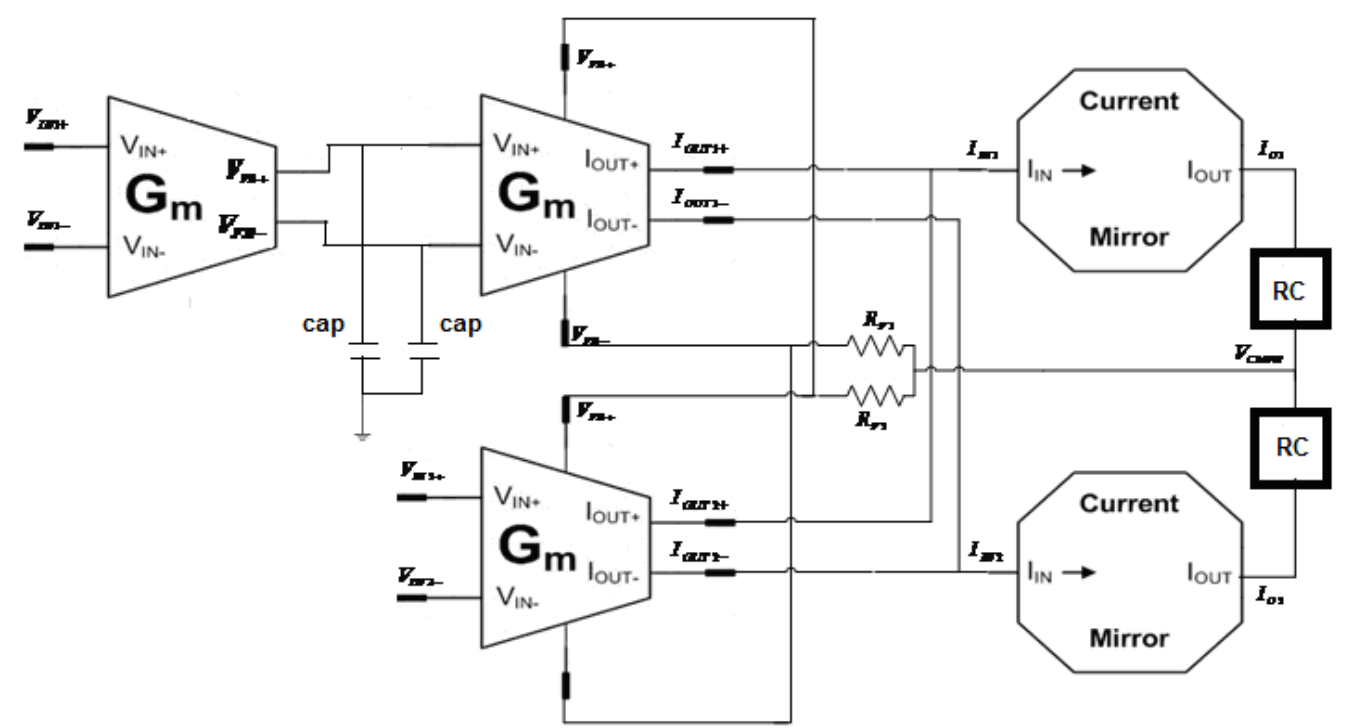

Figure 4.11: Readout circuit Final Design

The readout circuit consists of Operational Transconductance Amplifiers, Current Mirrors and $\mathrm{RC}$ filters.

It should be mentioned here that the circuit would function in the same manner if the mirror pair is removed. In this case the current gain becomes equal to zero and the common-mode rejection ratio of the OTA is not infinite. The input terminal of the current mirror is a low impedance node, this terminal sinks the common-mode current and generates a commonmode feedback voltage.

The first input of the circuit is the coil's output and the second input is the Hall sensor's output signal. The Hall sensor response can be described as a low pass filter [1] and the coil's response as a high pass filter [2]. The Hall sensor will also be referred to as magnetometer.

The Hall sensor's output voltage is calculated in equation (4.34)

$V_{\text {out }}=\frac{V_{\text {ref }}}{R_{1} \cdot C \cdot f_{\text {scan }}} \operatorname{tg} a=\frac{V_{\text {ref }}}{R_{1} \cdot C \cdot f_{\text {scan }}} \frac{k_{b b} \cdot I_{\text {meas }}}{B_{\text {ref }}}$

where $V_{r e f}$ is externally supplied reference voltage, $k_{b b}$ is the constant relating the measured current (bus bar constant) $I_{\text {meas }}$ and the magnetic field generated $B_{\text {meas }}$. Parameters $R_{1}$ and $\mathrm{C}$ define the nonlinear integrator and $f_{\text {scan }}$ is the scanning frequency [3]. According to the sampling theorem scanning frequency should be at least two times greater than the measuring frequency. 
The pick-up coil for high frequency range gives a signal proportional to $2 \cdot \pi \cdot k_{c} \cdot f_{\text {meas }}$, where $f_{\text {meas }}$ is the frequency of the measured current and $k_{c}$ is the coil's constant [3]. The equation that describes the coil's output voltage is calculated in (4.35)

$$
V_{\text {coil }}=-k_{c} \cdot S \cdot 2 \cdot \pi \cdot \mathrm{f}_{\text {meas }} \cdot I_{\text {meas }} \cdot \cos \left(2 \cdot \pi \cdot \mathrm{f}_{\text {meas }}\right)
$$

where $S$ is the surface of the coil and can be described by equation (4.36)

$S=N \cdot A$

where $\mathrm{N}$ is the number of the coil's turns and $\mathrm{A}$ is the area of each turn. Also, the current $I_{\text {meas }}$ can be calculated according to equation (4.37)

$$
I_{\text {meas }}=I_{\text {meas }(\max )} \cdot \sin \left(2 \cdot \pi \cdot \mathrm{f}_{\text {meas }}\right)
$$

Substituting the following equation (4.38)

$B_{\text {meas }}=k_{b b} \cdot I_{\text {meas }}$

In equations (4.34) and (4.35), equations (4.39) and (4.40) can be easily derived

$$
\begin{aligned}
& V_{\text {out }}=\frac{V_{\text {ref }}}{R_{1} \cdot C \cdot f_{\text {scan }}} \frac{B_{\text {meas }}}{B_{\text {ref }}} \\
& V_{\text {coil }}=-\frac{k_{c}}{k_{b b}} \cdot S \cdot 2 \cdot \pi \cdot \mathrm{f}_{\text {meas }} \cdot B_{\text {meas }} \cdot \cos \left(2 \cdot \pi \cdot \mathrm{f}_{\text {meas }}\right)
\end{aligned}
$$

The merging circuit is designed in order to add these two signals and get at the output a flat signal up to $1 \mathrm{MHz}$. Also, according to the output measured voltage (calculated in (4.35)) the magnetic field $B_{\text {meas }}$ is calculated.

According to equation (4.39) the magnetometer's output voltage response encounters a pole at frequency equal to $f_{o}=\frac{1}{2 \cdot \pi \cdot R_{1} \cdot C}$. This response is a low pass frequency response and the corner frequency is measured at about $30 \mathrm{KHz}$ [3]. According to equation (4.40) the coil has a highpass frequency response.

The RC filters are added at the outputs of the system in order to introduce cut-off frequency at $1 \mathrm{MHz}$. This is done according to the requirements of the system function, which is flat output frequency response up to $1 \mathrm{MHz}$.

The merging circuit adds these two signals, the signal from the magnetometer and the signal from the coil.

\subsubsection{Frequency Response}

The use of the circuit is as a wide-band magnetometer for current sensing applications. The frequency response block diagram of the circuit is presented in Figure 4.11. 
Galvanically Isolated, Wide-Band Current Sensors

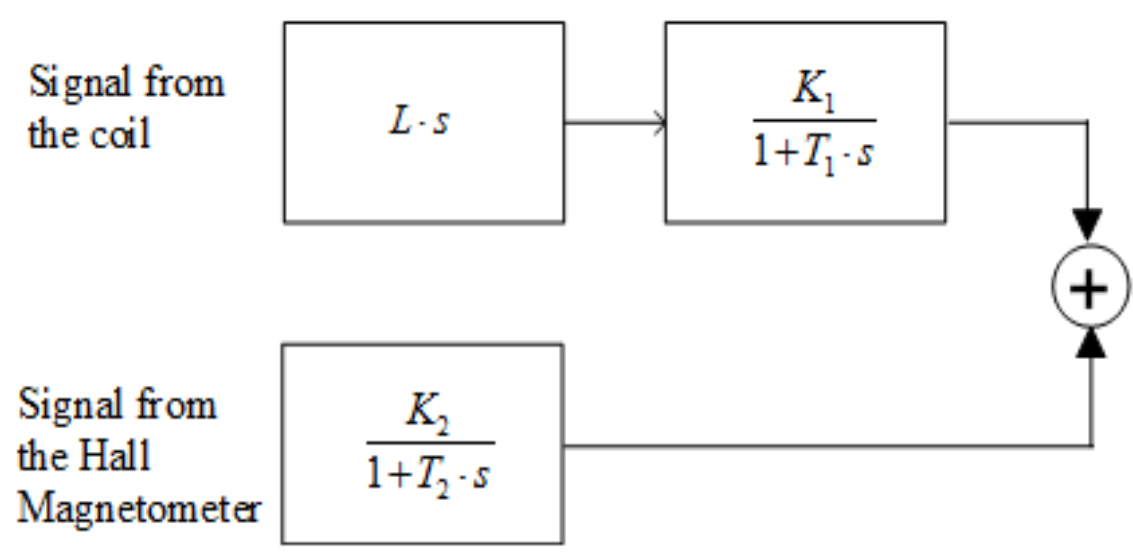

Figure 4.12: Frequency Block Diagram

In Figure 4.11, is presented the frequency block diagram of the fabricated final chip. The signal from the coil is described by a high pass frequency response equation and at this response, a pole is introduced. The signal from the Hall magnetometer can be described by a low pass frequency response equation. At the final chip, an $\mathrm{RC}$ filter is connected externally to the output, as it is presented in Figure 4.10. The values of the RC filter components are the same for both outputs. Thus, adding these filters to the frequency block diagram of Figure 4.11, the final diagram is presented in Figure 4.12.

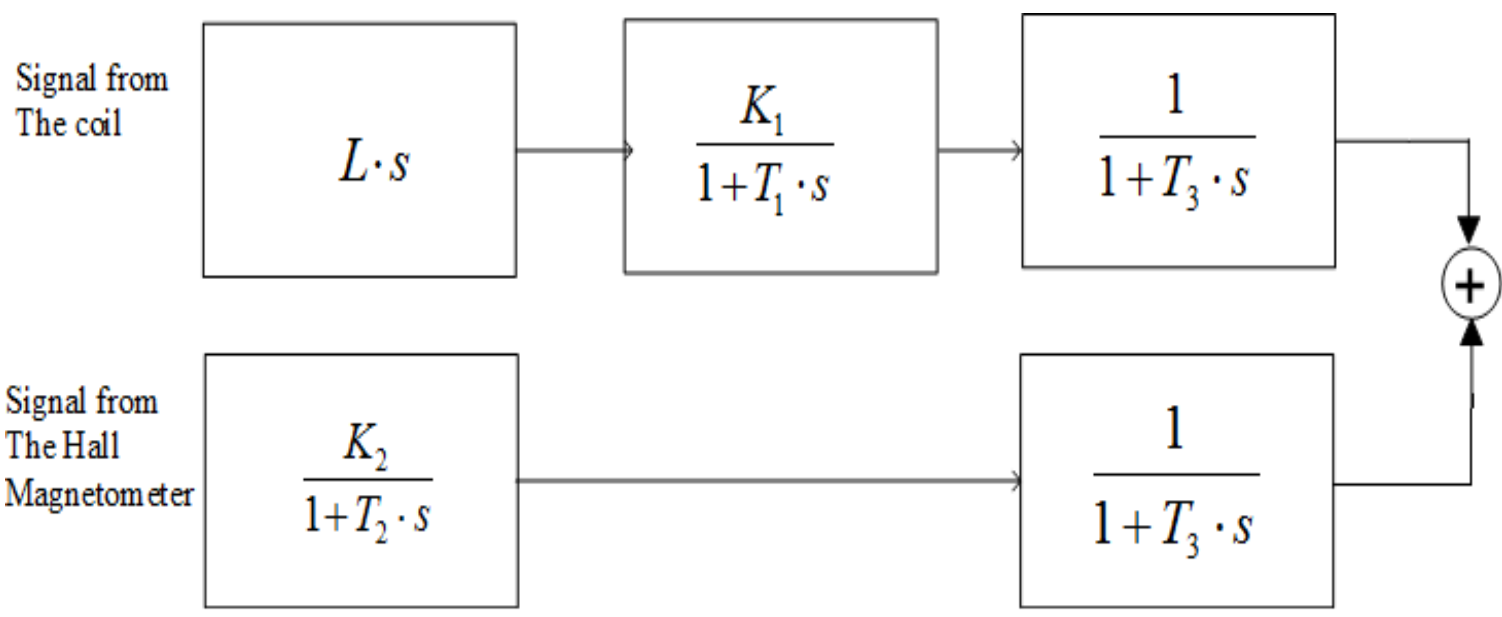

Figure 4.13: Final Frequency Block Diagram

In Figures 4.11 and 4.12, the signals are described by general form equations in the frequency domain. In Figure 4.13 the actual implementation of the final frequency block diagram is presented, including the actual frequency response equations. 
Galvanically Isolated, Wide-Band Current Sensors

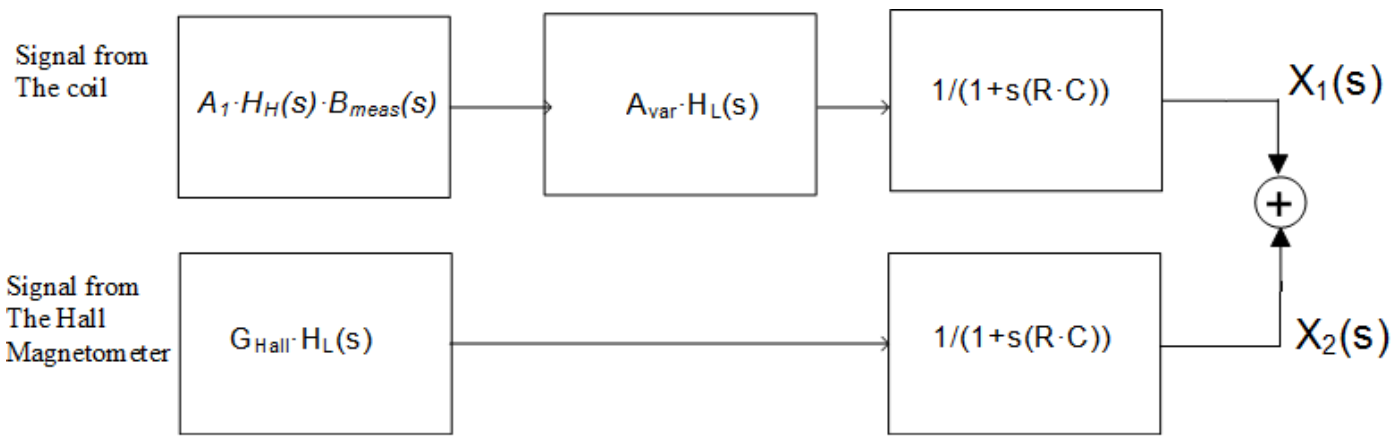

Figure 4.14: Actual implementation of the Frequency Block Diagram

In Figure 4.13 there are equations that describe the system's signals and will be explained below. Equation (4.41) describes the signal from the coil, where $A_{1}$ is the gain of the coil and is proportional to $\frac{k_{c}}{k_{b b}}, S$ and $B_{\text {meas }}$. $H_{H}(s)$ describe the high pass response of the coil. $A_{1} \cdot H_{H}(s) \cdot B_{\text {meas }}(s)$

Equation (4.42) represents the coil's signal at the output of the amplifier that is added at the system in order to introduce a pole with varying amplitude and varying cut-off frequency at the frequency domain response.

$A_{\mathrm{var}} \cdot H_{L}(s)$

Where $A_{\text {var }}$ stands for the (externally) varying gain which is introduced at this point of the system and $H_{L}(s)$ describe the low pass filter which is introduced. Its cutoff frequency is controlled by the values of the capacitors (cap), which are presented in Figure 4.10.

$G_{\text {Hall }} \cdot H_{L}(s)$

Equation (4.43) describes the magnetometer's signal, where $G_{\text {Hall }}$ is the gain of the Hall low frequency channel and is given by equation (4.44) [4] and $H_{L}(s)$ refers to the pole that is introduced by the magnetometer [3].

$G_{\text {Hall }}=\frac{V_{\text {ref }}}{B_{\text {ref }}} \cdot B_{\text {meas }}(s)$

Equation (4.45) describes the RC filter's response, where R, C refer to the values of the resistor and capacitor of the filter.

$\frac{1}{1+s(R \cdot C)}$

In the actual implementation of the system, the first output signal $X_{1}(s)$ of Figure 4.13 can be described by equation (4.46). The second output signal $\mathrm{X}_{2}(\mathrm{~s})$ can be described by equation (4.47), respectively. These two output signals are added at the output of the system, as it is also presented in Figure 4.13. 
Galvanically Isolated, Wide-Band Current Sensors

$$
\begin{aligned}
& X_{1}(s)=\left(A_{1} \cdot H_{H}(s) \cdot B_{\text {meas }}(s)\right) \cdot\left(A_{\mathrm{var}} \cdot H_{L}(s)\right) \cdot\left(\frac{1}{1+s(R \cdot C)}\right) \\
& X_{2}(s)=\left(\frac{V_{\text {ref }}}{B_{\text {ref }}} \cdot B_{\text {meas }}(s) \cdot H_{L}(s)\right) \cdot\left(\frac{1}{1+s(R \cdot C)}\right)
\end{aligned}
$$

The output of the merging circuit can be described by equation (4.48)

$$
\begin{aligned}
& X(s)=X_{1}(s)+X_{2}(s) \\
& X(s)=\left(\frac{1}{1+s(R \cdot C)}\right)\left[\left(A_{1} \cdot H_{H}(s) \cdot B_{\text {meas }}(s)\right) \cdot\left(A_{\mathrm{var}} \cdot H_{L}(s)\right)+\left(\frac{V_{\text {ref }}}{B_{\text {ref }}} \cdot B_{\text {meas }}(s) \cdot H_{L}(s)\right)\right] \\
& X(s)=\left(\frac{1}{1+s(R \cdot C)}\right)\left[\left(A_{1} \cdot H_{H}(s)\right) \cdot\left(A_{\mathrm{var}} \cdot H_{L}(s)\right)+\left(\frac{V_{\text {ref }}}{B_{\text {ref }}} \cdot H_{L}(s)\right)\right] \cdot B_{\text {meas }}(s)
\end{aligned}
$$

By inspection of equation (4.48), $B_{\text {meas }}$ can be calculated if the poles and the zeros are matched in order to achieve flat output frequency response.

According to simulation and measurement results that are presented below, this can be done in order to achieve flat output frequency response.

\subsection{Readout Circuit Design}

The design of the Readout Circuit is done according to the following tradeoffs:

- Amplifier's Gain, having also in mind that the circuit is connected in feedback topology. Thus there is also a frequency-amplitude tradeoff.

- Small area of the final chip.

- Restrictions that may arise according to the inputs, the coil's and the magnetometer's signals.

The Basic OTA gain is chosen in order to have adequate signal amplification, avoid oscillations and fulfill the input signals restrictions.

The OTA's gain is calculated according equation (4.49)

$$
\mathrm{G}_{\mathrm{m}}=\mathrm{V}_{\mathrm{o}} /\left(\mathrm{V}_{\mathrm{i}} \cdot \mathrm{R}_{\mathrm{L}}\right)
$$

Where $G_{m}$ is the OTA's gain, $V_{o}$ and $V_{i}$ are the output and input voltage signals respectively and $R_{L}$ is a resistor that is connected to the output for calculation purpose. Here $G_{m}=1$. This selection is done in order to be sure that no oscillations will occur, since the system is 
Galvanically Isolated, Wide-Band Current Sensors

connected in feedback loop and consists of coil and resistors. Besides that, in order to compare it with a similar design [3], also the output gain that is described there is low.

The gain is mainly proportional to the size of the transistors and in accordance to the current. There is a limitation in the total layout size of the design. The layout size is proportional to the size of the transistors. So, there is tradeoff between the gain and the size. The size that is chosen is considered the most convenient in order to have enough gain, small overall design size and avoid the possibility of oscillation. The OTAs used at this design should and do have enough phase margin in order to be sure that the circuit will not oscillate since it is connected in feedback configuration.

Also, an important parameter is the restriction that may arise according to the signals at the inputs of the design. During the design, the input's performance and signals was taken into account.

It is important here to highlight the fact that compensation resistors and capacitors cannot be used because the flat frequency response at the output will be changed and as it has already been described the poles and zeros of the circuit are really crucial to the output response. 
Galvanically Isolated, Wide-Band Current Sensors

\section{References}

[1] Nicolas Karrer, Patrick Hofer-Noser, A new current measuring principle for power electronic applications,(1999) 279.

[2] Jing Xu, Lingyin Zhao, Zhenxian Liang, J.D. van Wyk, Design of an Embedded Bi-planar Coil-Based Integrated Current Sensor for Power Module Integration, (2005) 369-374

[3] M.Banjevic, F.Liakou, B.Furrer, S.Dimitrijevic, M.Blagojevic, P.D.Dimitropoulos and R.S.Popovic, Open-loop CMOS Current Transducer with Low Temperature Cross-sensitivity, Eurosensors XXV, 2011

[4] M.Banjevic, High Bandwidth CMOS Magnetic Sensors Based on the Miniaturized Circular Vertical Hall Device, PHD thesis. 


\section{Simulation \& Measurement Results}

\subsection{Simulation Results}

The design of the circuit and the simulation results that are presented here are simulated with Cadence tool [1]. The design process is austriamicrosystems [2] AMS 0.35, H35B4D3, High Voltage. The reason for this choice is that this design is a part of a bigger one which incorporates Hall sensor and the Hall sensor had to be developed in this process. The choice of this process was also convenient for this design part.

\subsubsection{Input models}

In order to simulate the system, the first step was to model the inputs of the system. Thus, a model for the flat coil is designed as the first input and a model for the Hall sensor's response is designed as the second input. The Hall sensor hereafter will be also called as magnetometer. These models are designed in accordance to the measurements and response results of the flat pick-up coil and the Hall magnetic sensor.

The coil and the Hall sensor measurements are based on a magnetic field and are proportional to a corresponding voltage. Thus, in order to design the models, there is the same voltage at the input of the coil and the magnetometer which stands for the magnetic field measurement. Besides that, the Hall sensor and the coil have a specific gain. These gain values are also included in the Cadence models that are designed. These values are calculated according to the flat pick-up coil report [3] and to the magnetometer's response. The models that have been designed in Cadence are presented in Figure 5.1. There is a common input voltage source at the input of the two models. At the upper part of Figure 5.1 the coil's model is presented and below is the Hall sensor's model, respectively. By inspection of Figure 5.1, the parasitic resistance and capacitance of the coil are included in the model design. The values are those that have been measured in [3]. Also, the Hall sensor is modeled as an RC filter, according to the report of the Hall sensor's designer [4]. 


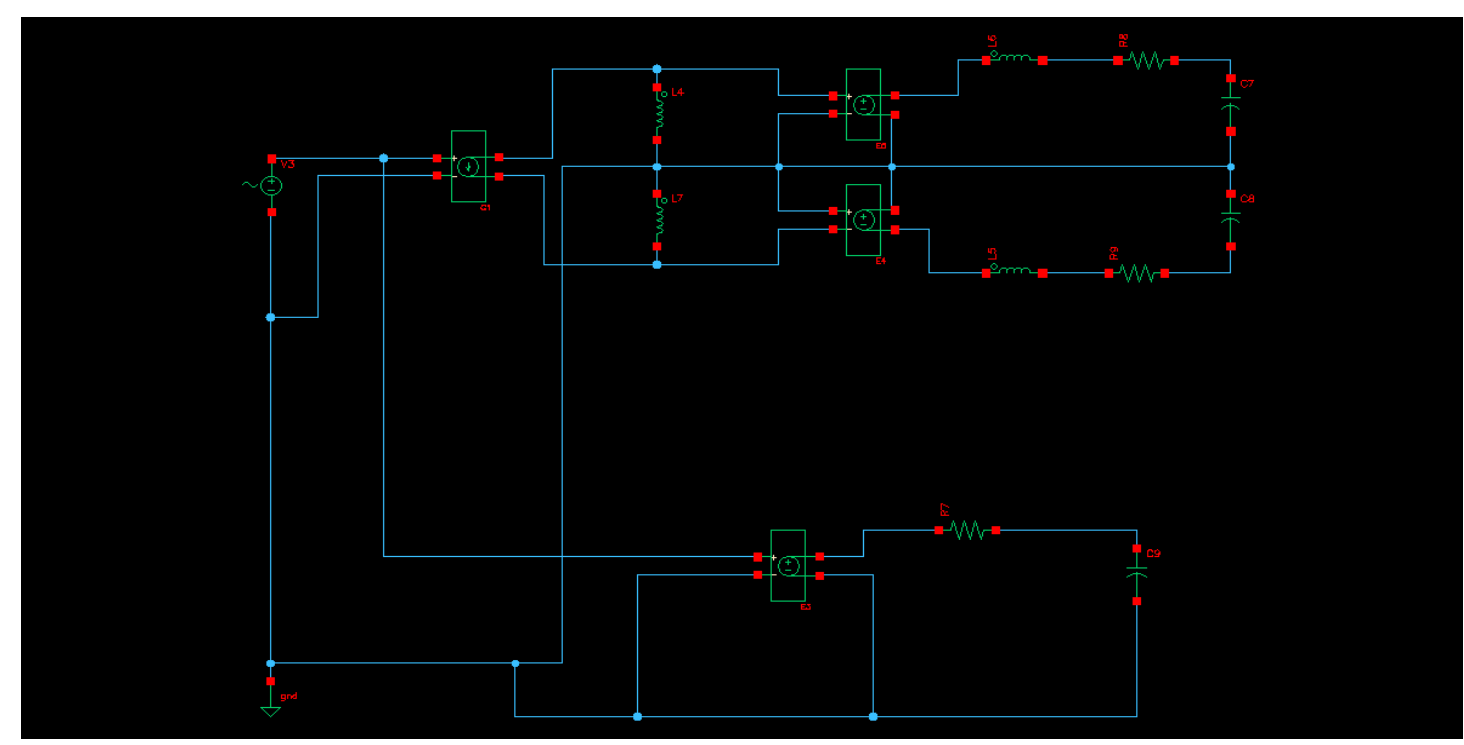

Figure 5.1: Cadence schematic of input models

\subsubsection{Pick-up Coil}

A pick-up coil can measure a current waveform according to the change of the magnetic flux. A coil which has a couple of turns can be used as a sensor, having also the advantage of a compact and simple structure. According to the report for the flat pick-up coil [3] using equations (5.1)-(5.4) the induced voltage can be calculated.

$U_{\text {pickup }}=-\frac{d \Phi}{d t}=-S \frac{d B}{d t}$

Where $\mathrm{S}$ is the total surface, $B=B_{\max } \cdot \sin \omega t$

$U_{\text {pickup }}=-S B_{0} \omega \cos \omega t$

$U_{\text {pickup }_{\max }}=S B_{\text {max }} \omega=N A B_{\text {max }} 2 \pi f$

For the coil that is used here the numbers of turns are 4 and the surface of each turn is $5 \mathrm{~mm}^{2}$.

According to the flat-coil report [3] $L=0.2 u H$ and $R=0.2 \mathrm{Ohm}$. At the model that is presented in Figure 5.1, there are also two capacitors in order to model the parasitic capacitance of the coil. As it is presented in Figure 5.1, there is an input voltage reference source. The input current of the pick-up coil is modeled as a controlled voltage current source. The gain of this current source is calculated in accordance to the results of the pick-up coil report [3].

\subsubsection{Hall Sensor - Magnetometer}

The Hall sensor's response is modeled as an RC filter with the corner frequency measured at about $30 \mathrm{KHz}[4,5]$. There is a controlled voltage, voltage source at the input of the RC filter in order to model and include the magnetometer's gain. 
Galvanically Isolated, Wide-Band Current Sensors

\subsubsection{Simulation Results with modeled inputs}

In Figure 5.2 the simulation results for the two inputs are presented in the frequency domain. On the right part of Figure 5.2, the coil's response is presented and on the left part the RC filter's (Hall sensor's) response is presented, respectively. By inspection of Figure 5.2, the coil's frequency response is a high-pass response while the Hall sensor's response is a lowpass frequency response.
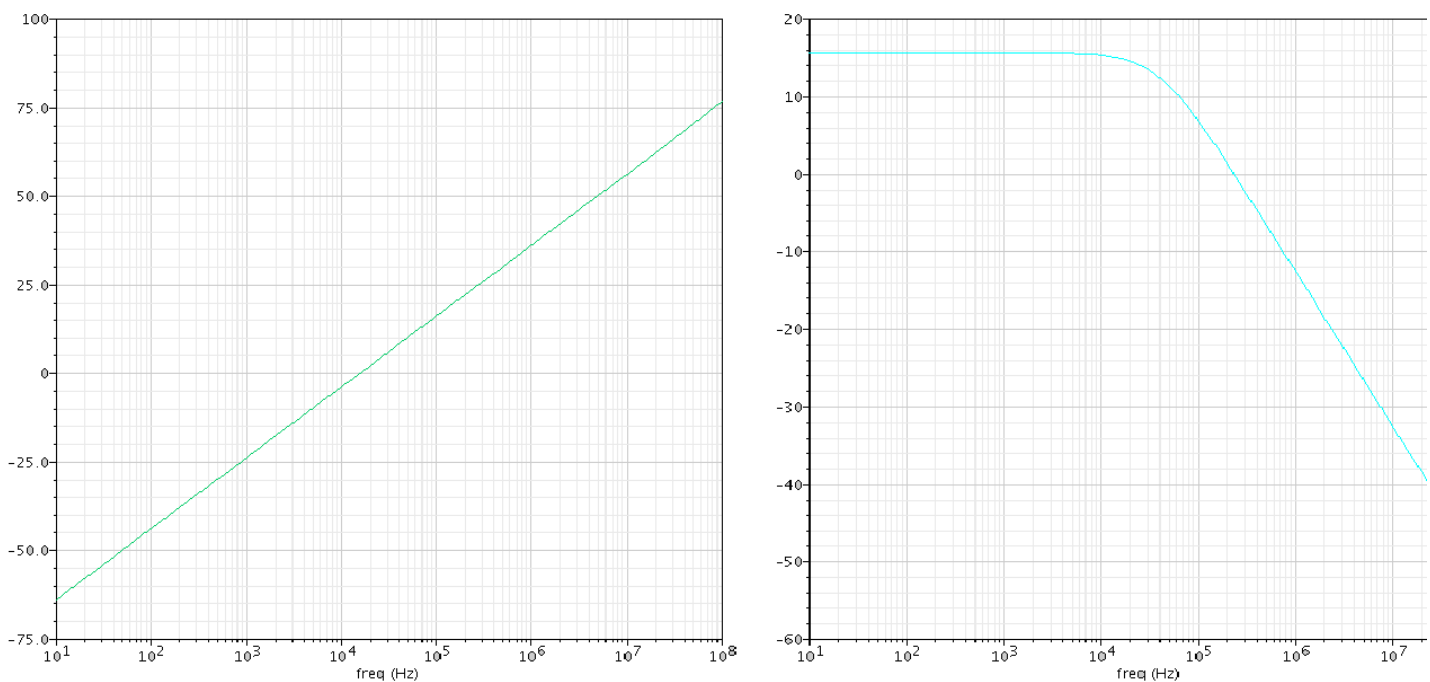

Figure 5.2: Input models' Frequency Response
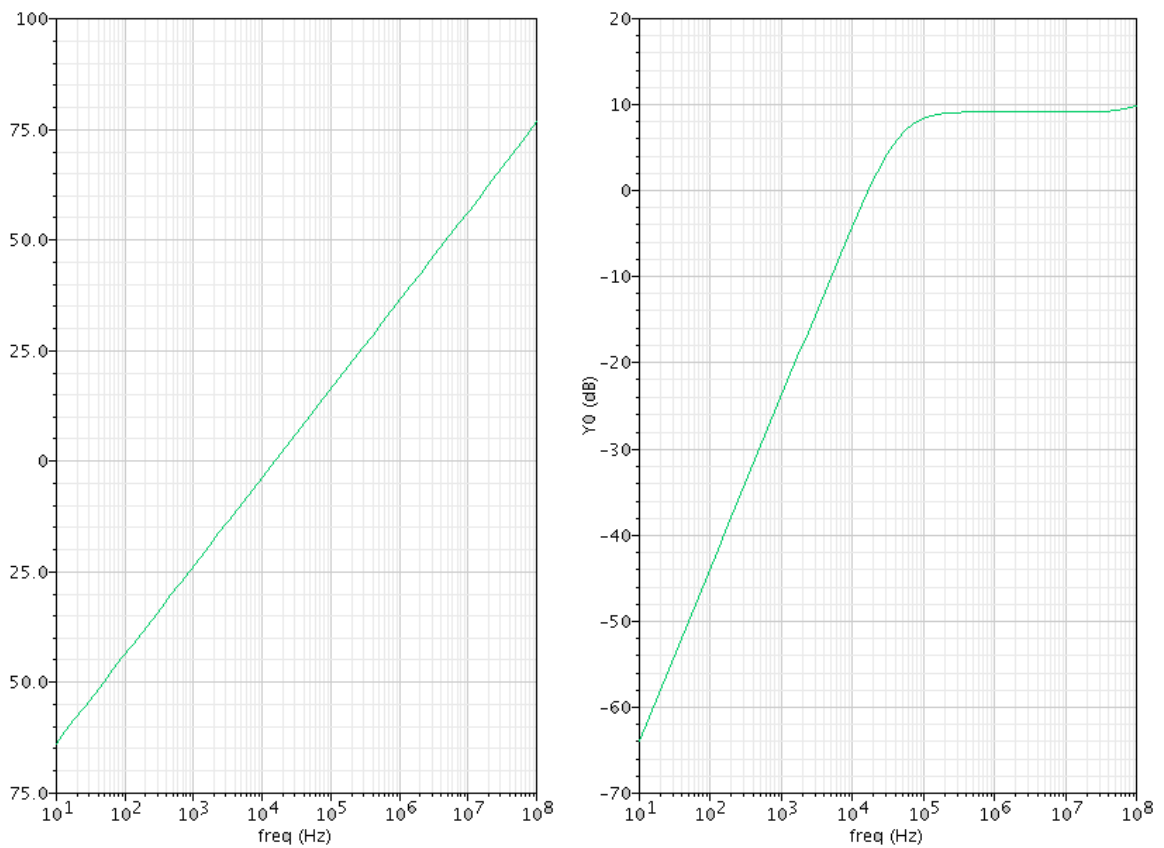

Figure 5.3: Coil's response 
As it has already been described, a pole is introduced into the coil's response. The frequency response of the coil, before and after the influence of the pole is presented in Figure 5.3, the figures on the left and on the right part respectively. This is done in order to be able to add the signal from the coil and the signal from the Hall sensor in order to get a flat output frequency response. When the parameters of the system are chosen in order to match the pole and amplitude from the coil and the pole and the amplitude from the magnetometer, the frequency response at the output is flat.

The system is designed in order to be able to change the corner frequency and the amplitude of the coil's response that is presented in Figure 5.3 and thus match the two signals at the output. The corner frequency can be changed by changing the values of the capacitors (cap) of the readout circuit (described in Chapter 4). The amplitude can be changed by changing the $V_{S C O N}$ input voltage that is applied by the biasing circuit.

In Figure 5.4 the frequency response results of the input voltages and the output currents are presented, for different values of the capacitors (cap). It should also be highlighted here that the values of both capacitors are the same.
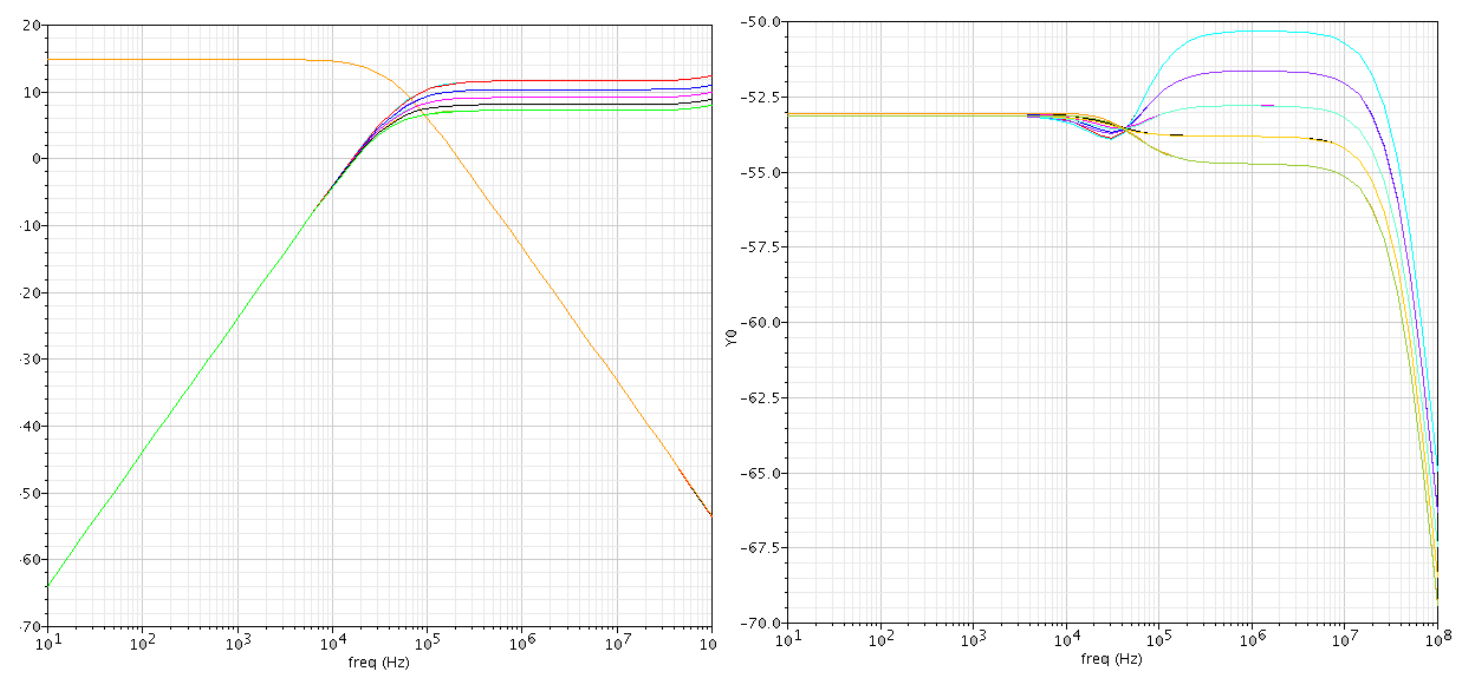

Figure 5.4: Input voltages and output current frequency response for varying cap values

As it is presented in Figure 5.4, for different capacitors values there are different corner frequencies. The low-pass response of the left part of Figure 5.4 is the magnetometer's response and the high pass response is the coil's response. As it is presented, the coil's response varies in accordance to varying capacitors values. Thus, the appropriate corner frequency can be adjusted and applied in order to match the signals and get flat output response. In Figure 5.5, the corresponding output voltage response for varying capacitors values is presented. 


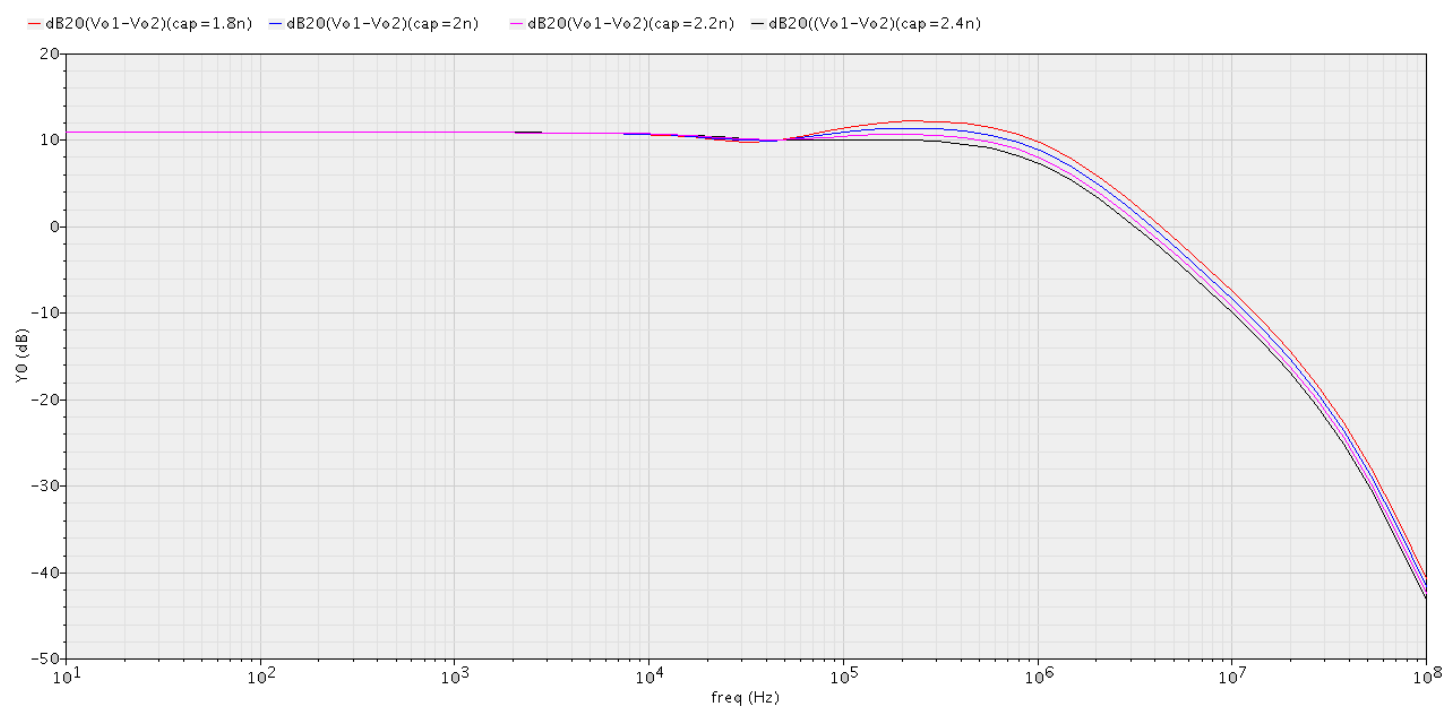

Figure 5.5: Output voltage frequency response for varying cap values
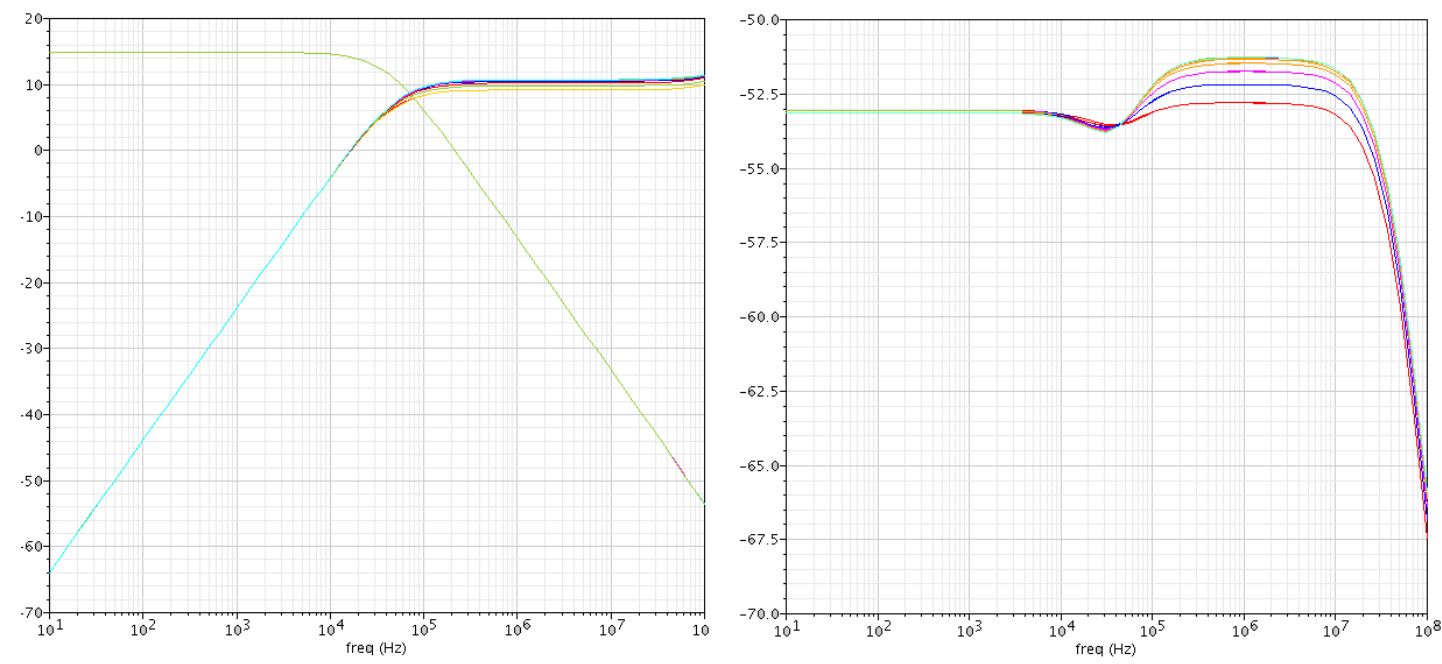

Figure 5.6: Input voltages and output current frequency response for varying amplitude values

In Figure 5.6 the frequency response results of the input voltages and the output currents are presented. Here, also the magnetometer's and the coil's response are presented on the left part of Figure 5.6, but in this case the OTA's gain exhibits different values. The OTA's gain can be controlled by the $V_{S C O N}$ voltage of the biasing circuit. For varying $V_{S C O N}$ values, varying biasing current values and varying OTA's gain values are achieved and in accordance varying amplitude signal values. The appropriate value of the OTA's gain and the $\mathrm{V}_{\text {SCON }}$ voltage can be chosen and applied in order to match the signals and get flat output frequency response. In Figure 5.7, the corresponding output voltage response for varying $V_{\text {ScON }}$ voltage values is presented. 
Galvanically Isolated, Wide-Band Current Sensors

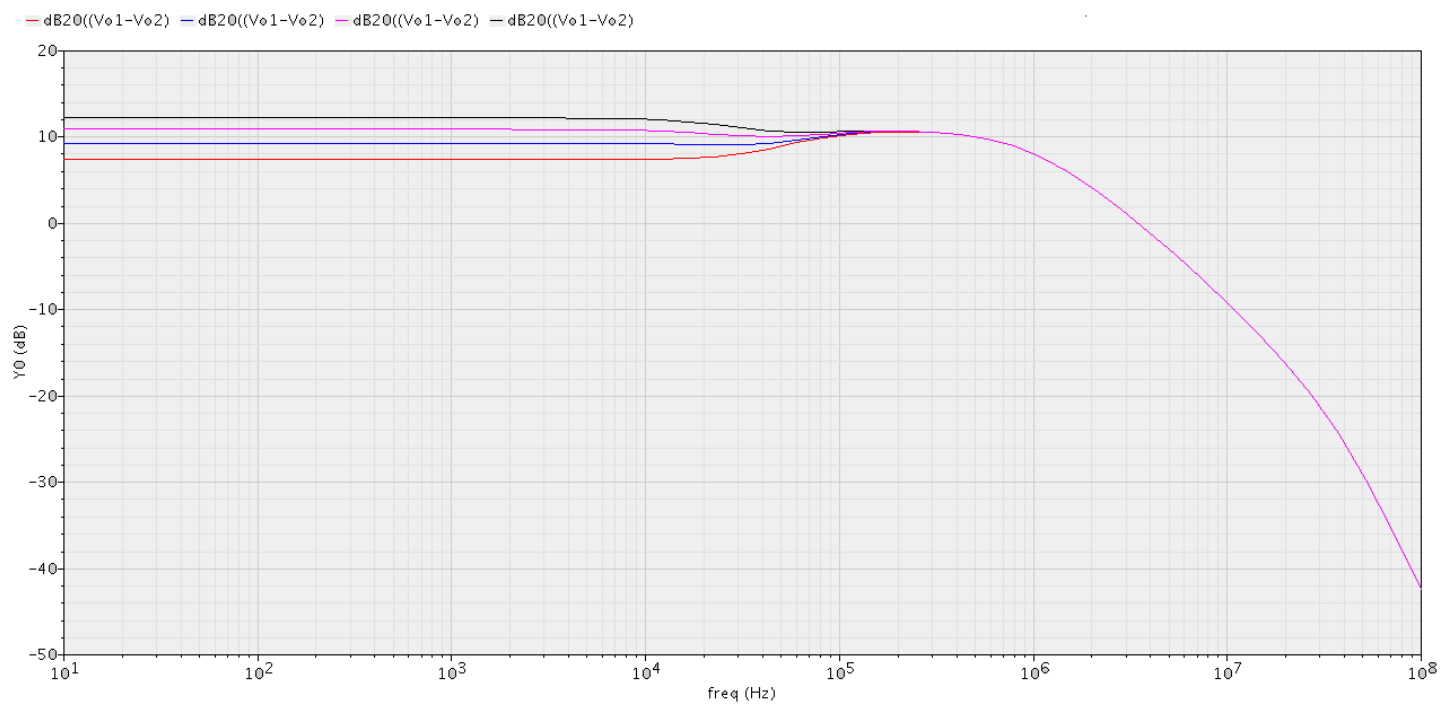

Figure 5.7: Output voltage frequency response for varying amplitude values

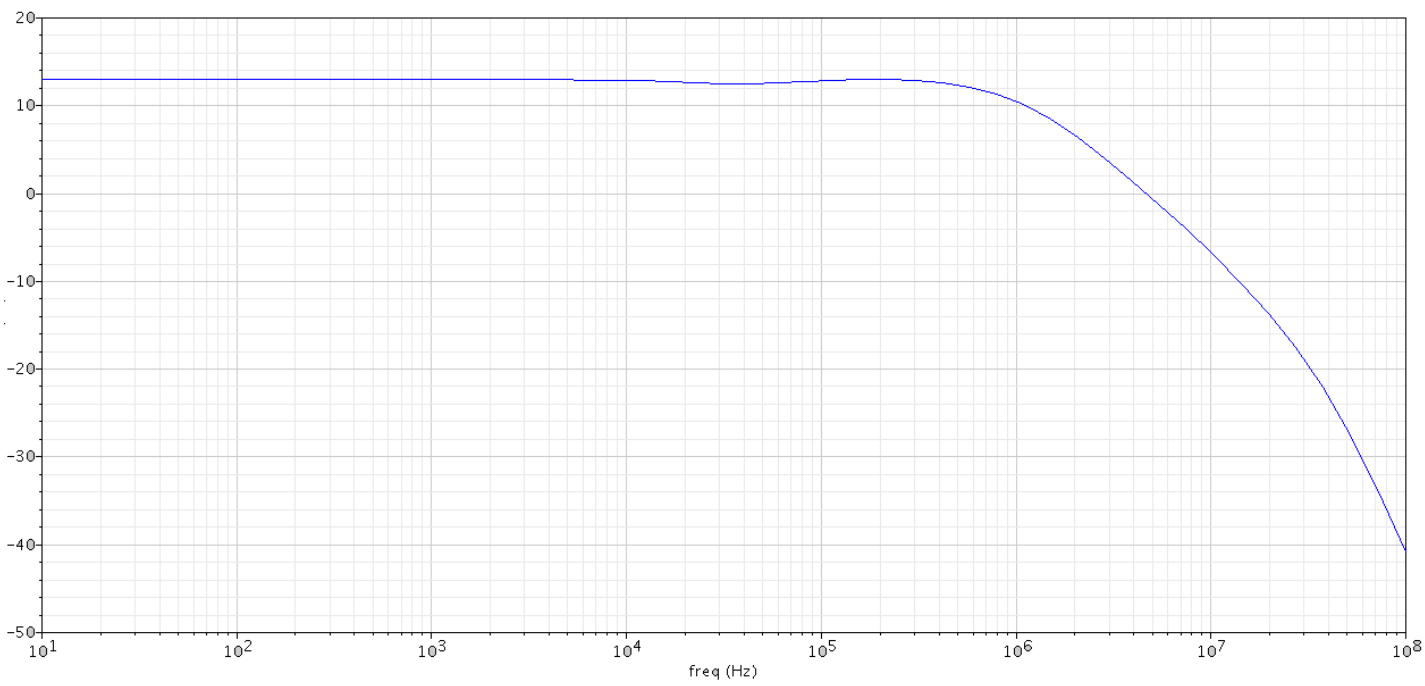

Figure 5.8: Voltage output frequency response

In Figure 5.8, the chosen voltage output frequency response of the readout circuit is presented. That response is the result of the subtraction of the output voltages. The RC filter at the output introduces a pole at $1 \mathrm{MHz}$, as it can be also verified by the response of Figures $5.5,5.7$ and 5.8 .

The transient response of the coil at the first input is presented in Figure 5.9. The coil's transient response is measured for various frequency values equal to $1 \mathrm{KHz}, 10 \mathrm{KHz}, 100$ $\mathrm{KHz}$ and $1 \mathrm{MHz}$. 
Galvanically Isolated, Wide-Band Current Sensors

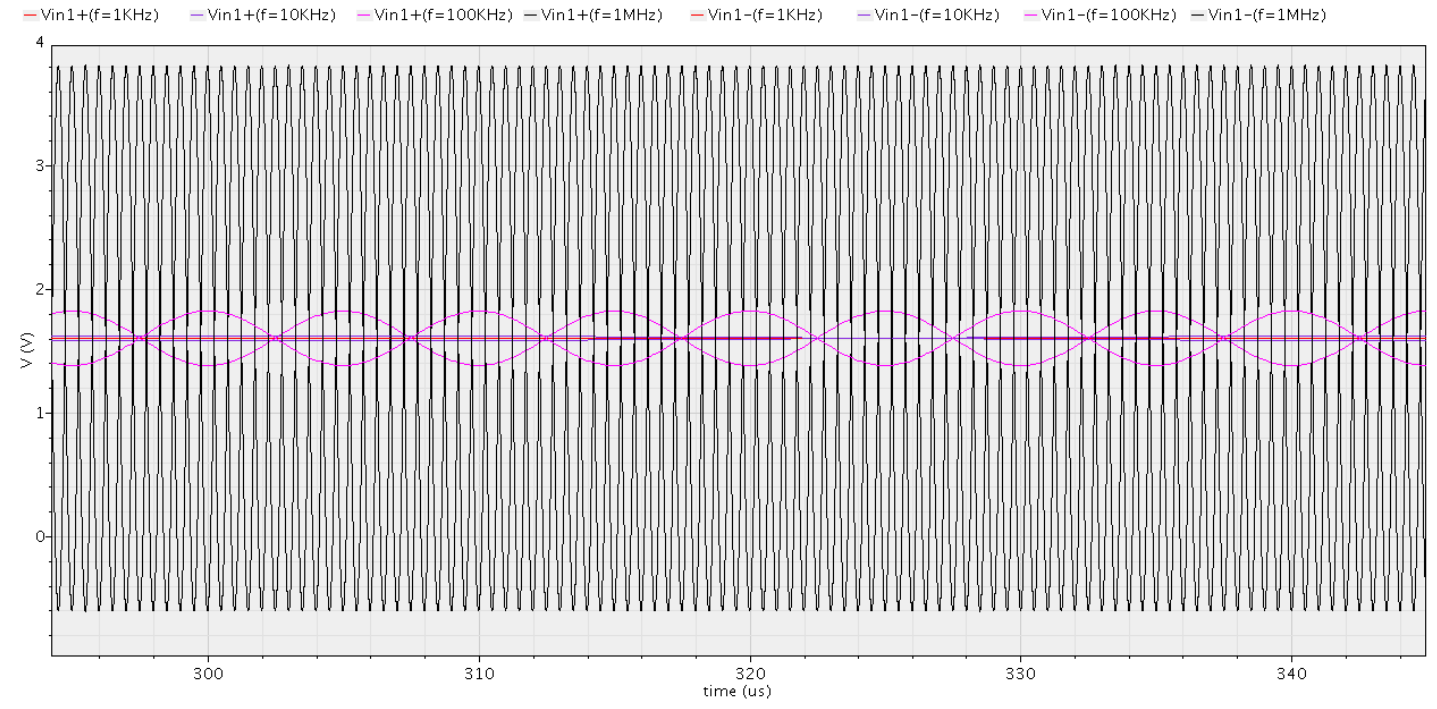

Figure 5.9: Coil input (transient response)

In Figure 5.10, the transient response of the magnetometer's model at the second input is presented, for various frequency values equal to $1 \mathrm{KHz}, 10 \mathrm{KHz}, 100 \mathrm{KHz}$ and $1 \mathrm{MHz}$.

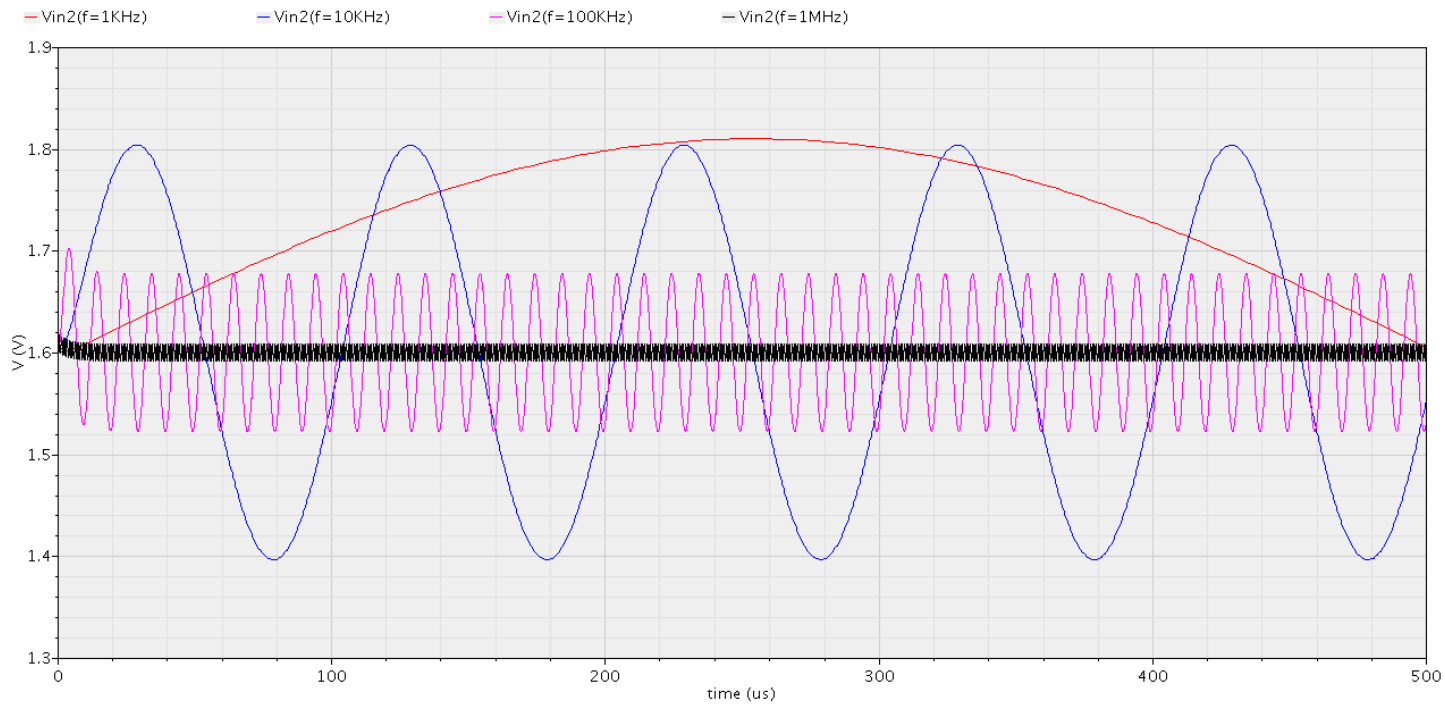

Figure 5.10: Magnetometer's model (transient response)

In Figure 5.11, the transient response of the current at the output is presented for frequency values equal to $30 \mathrm{KHz}, 35 \mathrm{KHz}, 40 \mathrm{KHz}$ and $45 \mathrm{KHz}$. These values are near the pole of the magnetometer which is calculated at frequency equal to $37 \mathrm{KHz}$, according to the magnetometer's design at this point. By inspection of Figure 5.11, the amplitude of the measured current at the output is the same, for the aforementioned frequency values. 


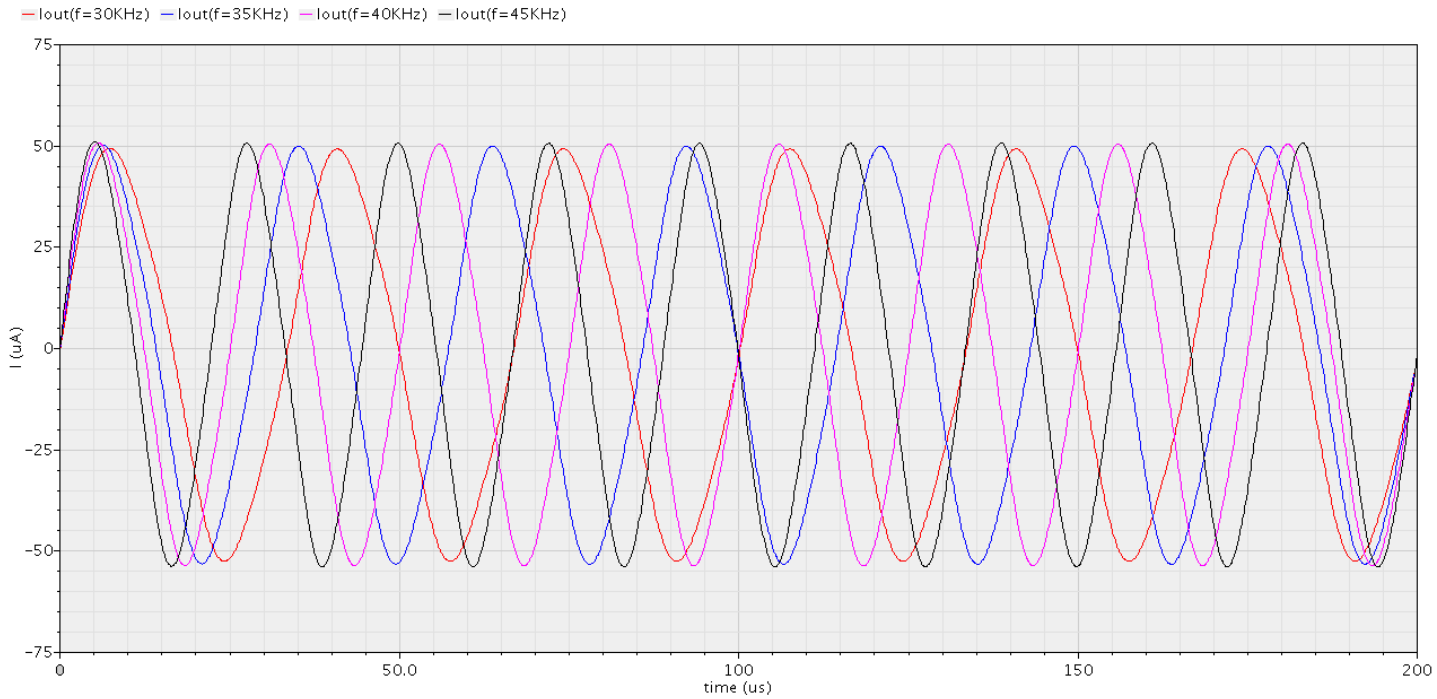

Figure 5.11: Output current transient response (low frequencies values)

In Figure 5.12, the transient response of the current at the output is presented for frequency values equal to $1 \mathrm{KHz}, 10 \mathrm{KHz}, 100 \mathrm{KHz}$ and $1 \mathrm{MHz}$. As it is presented in Figure 5.12 , the amplitude of the output signals is the same for frequencies lower than $1 \mathrm{MHz}$. The output signal's amplitude is lower at $1 \mathrm{MHz}$. The results of Figure 5.12 are in accordance and confirm the frequency response results.

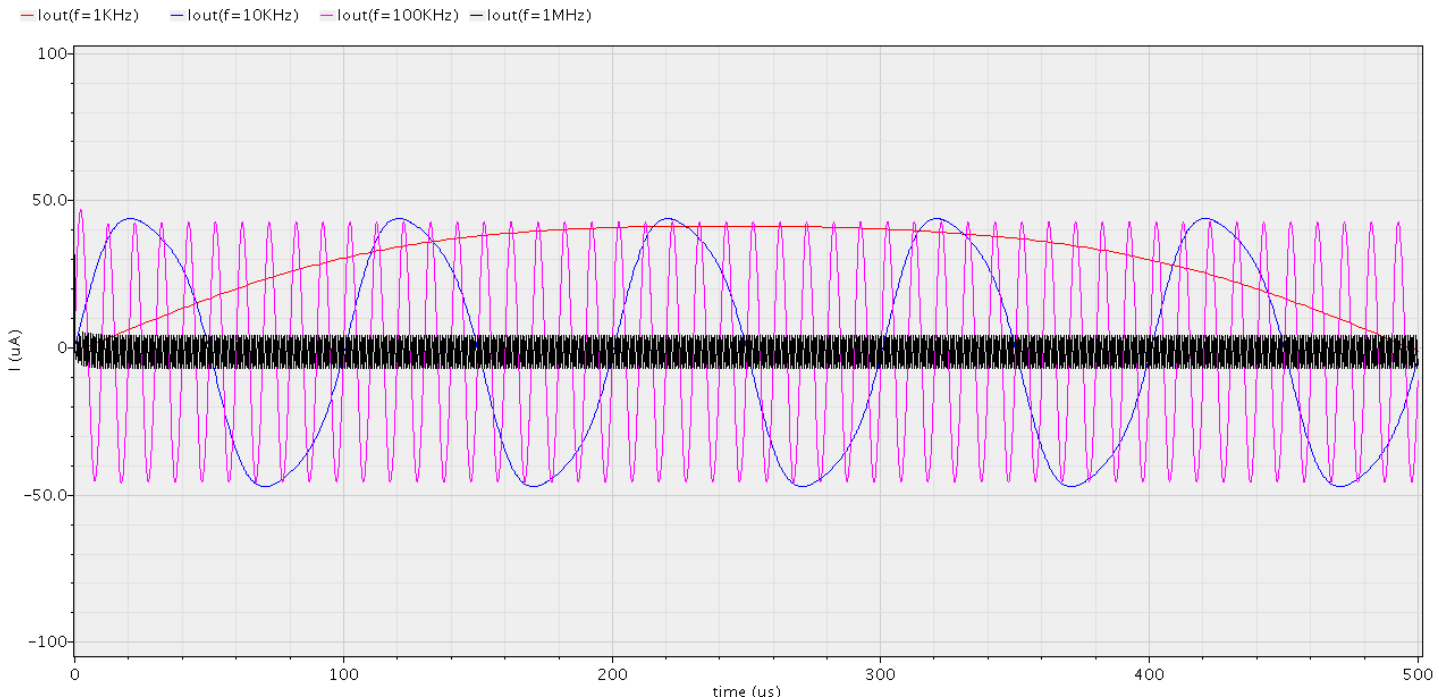

Figure 5.12: Output current transient response (high frequencies values)

In Figure 5.13, the output voltage transient response is presented for frequency values equal to $30 \mathrm{KHz}, 35 \mathrm{KHz}, 40 \mathrm{KHz}$ and $45 \mathrm{KHz}$. By inspection of Figure 5.13 , the amplitude of the measured current at the output is the same, for the aforementioned frequency values. 


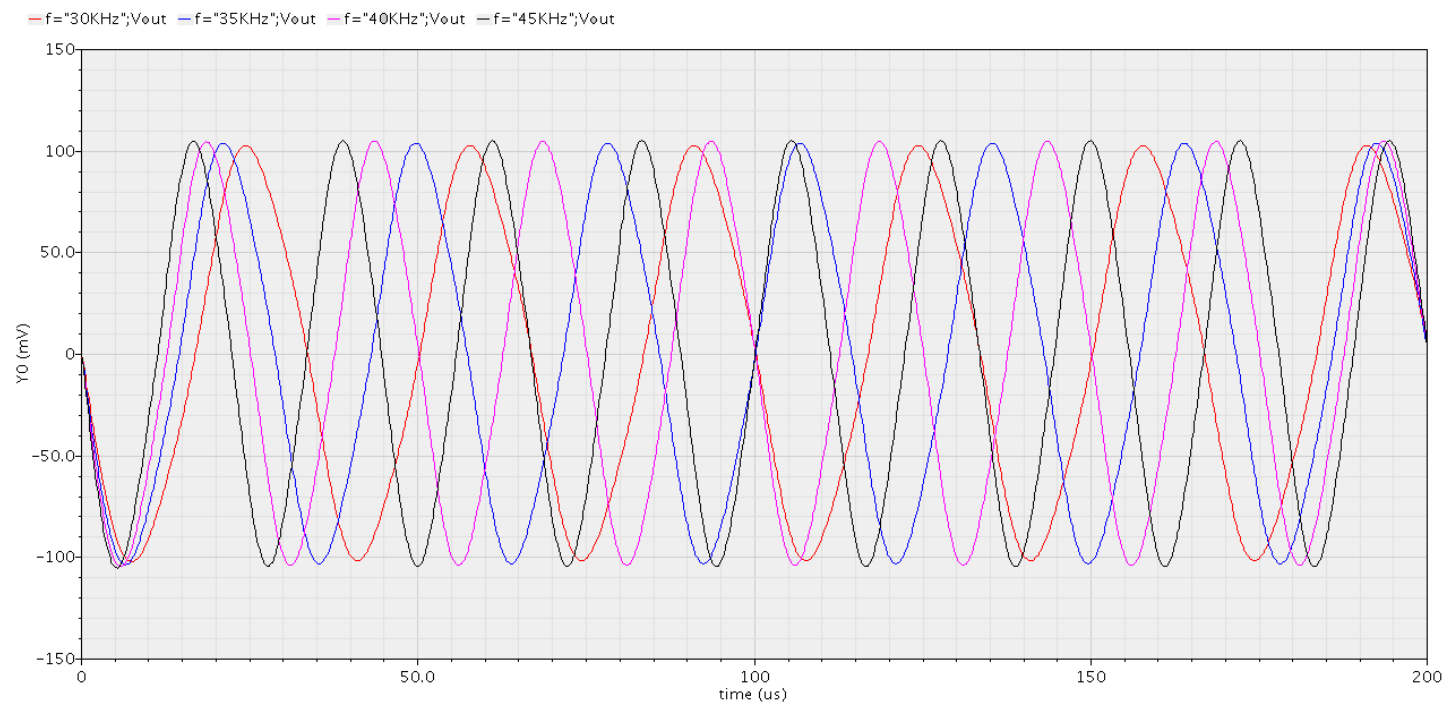

Figure 5.13: Output voltage transient response (low frequencies values)

In Figure 5.14 the output voltage transient response is presented for frequency values equal to $1 \mathrm{KHz}, 10 \mathrm{KHz}, 100 \mathrm{KHz}$ and $1 \mathrm{MHz}$. As it is presented, the amplitude of the output signals is the same for lower frequencies up to $1 \mathrm{MHz}$ and is reduced at $1 \mathrm{MHz}$. The results of Figure 5.14 are in accordance and confirm the frequency response results.

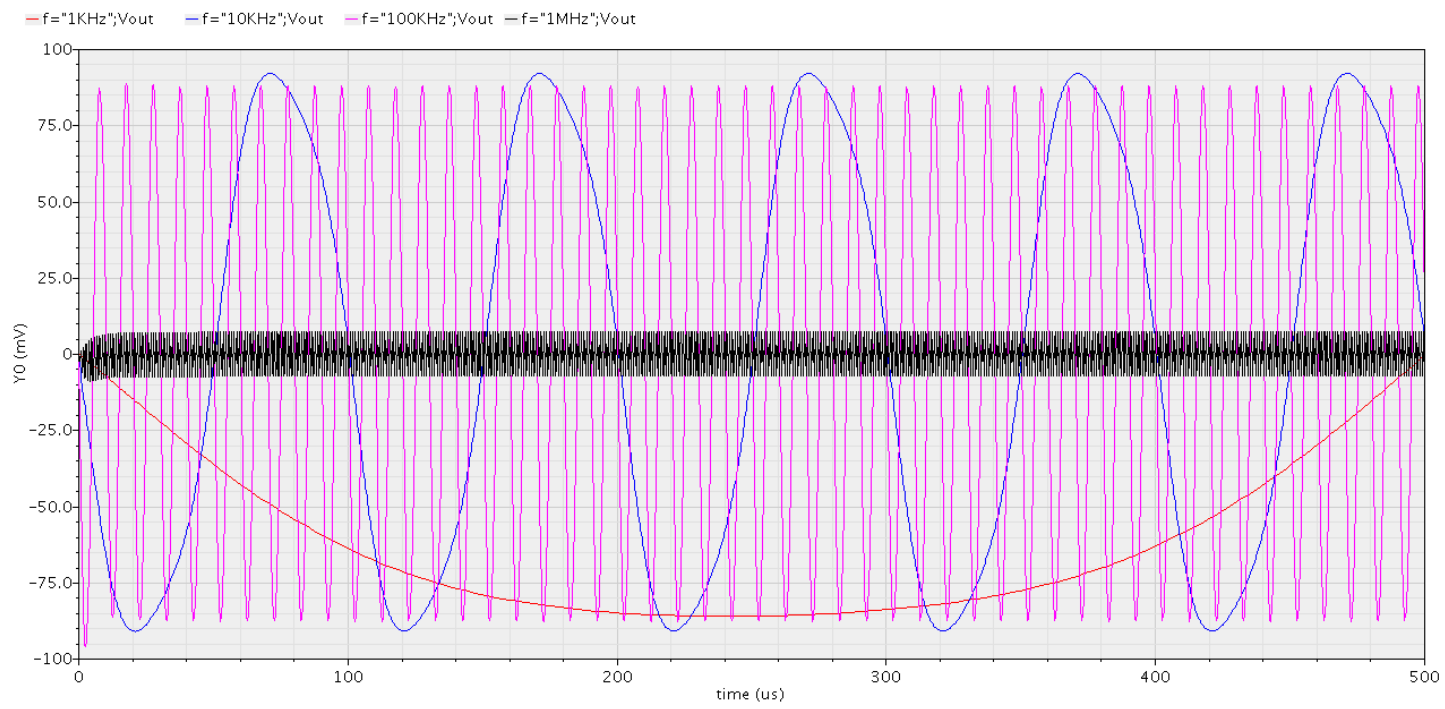

Figure 5.14: Output Voltage transient response (high frequencies values)

The parameters values for the above simulation results with models at the inputs are presented in Table 5.1

\begin{tabular}{|l|l|}
\hline$A_{\text {mag }}$ & 6 \\
\hline $\mathrm{V}_{\text {SCON }}$ & $1.4 \mathrm{~V}$ \\
\hline
\end{tabular}




\begin{tabular}{|l|l|}
\hline$R_{F}$ & 1 Ohm \\
\hline cap & $2.2 \mathrm{n}$ \\
\hline$R_{\text {out }}$ & 1 KOhm \\
\hline$C_{\text {out }}$ & $150 p$ \\
\hline
\end{tabular}

Table 5.1: Parameters values for modeled inputs

Where $A_{\text {mag }}$ stands for the magnetometer's gain and is used as a parameter at the voltage controlled voltage source. $V_{S C O N}$ is the input voltage that is applied at the biasing circuit. $R_{F}$ stands for the value of both the feedback resistors which have equal values. Cap is the value of the equal valued capacitors. $\mathrm{R}_{\text {out }}$ and $\mathrm{C}_{\text {out }}$ are the values of the resistance and capacitance of the RC filter which is connected at the output.

\subsubsection{Simulation Results with the Hall sensor circuit}

The Hall sensor's model is replaced by the Hall sensor circuit. The transient simulation results of the coil and Hall sensor transient response for frequency values equal to $1 \mathrm{KHz}, 10 \mathrm{KHz}$, $100 \mathrm{KHz}$ and $1 \mathrm{MHz}$ are presented in Figures 5.15 and 5.16, respectively. In this case there was not possible to simulate and plot frequency response (ac response) results. This is so, because the Hall sensor's circuit includes also digital components.

In Figures 5.17 and 5.18 the transient response of the output current and output voltage are presented for frequency values equal to $1 \mathrm{KHz}, 10 \mathrm{KHz}, 100 \mathrm{KHz}$ and $1 \mathrm{MHz}$. According to the results, the amplitude of the output signals is the same for lower frequencies up to $1 \mathrm{MHz}$ and falls at $1 \mathrm{MHz}$. These results are in accordance to the results that are plotted using a model for the magnetometer. Also, these results are in accordance to the design specifications.

The parameters values for the above simulation results are presented in Table 5.2. In this case, there is a parametric output voltage value of the Hall sensor and for varying values of that voltage the Hall sensor's amplitude can be adjusted. That parameter is a part of the Hall sensor and is not presented here in detail.

\begin{tabular}{|l|l|}
\hline$V_{\text {SCON }}$ & $1.4 \mathrm{~V}$ \\
\hline$R_{F}$ & 1 Ohm \\
\hline cap & $4 n$ \\
\hline
\end{tabular}


Galvanically Isolated, Wide-Band Current Sensors

\begin{tabular}{|l|l|}
\hline$R_{\text {out }}$ & 1 KOhm \\
\hline$C_{\text {out }}$ & $150 p$ \\
\hline
\end{tabular}

Table 5.2: Parameters values with the Hall sensor at the second input

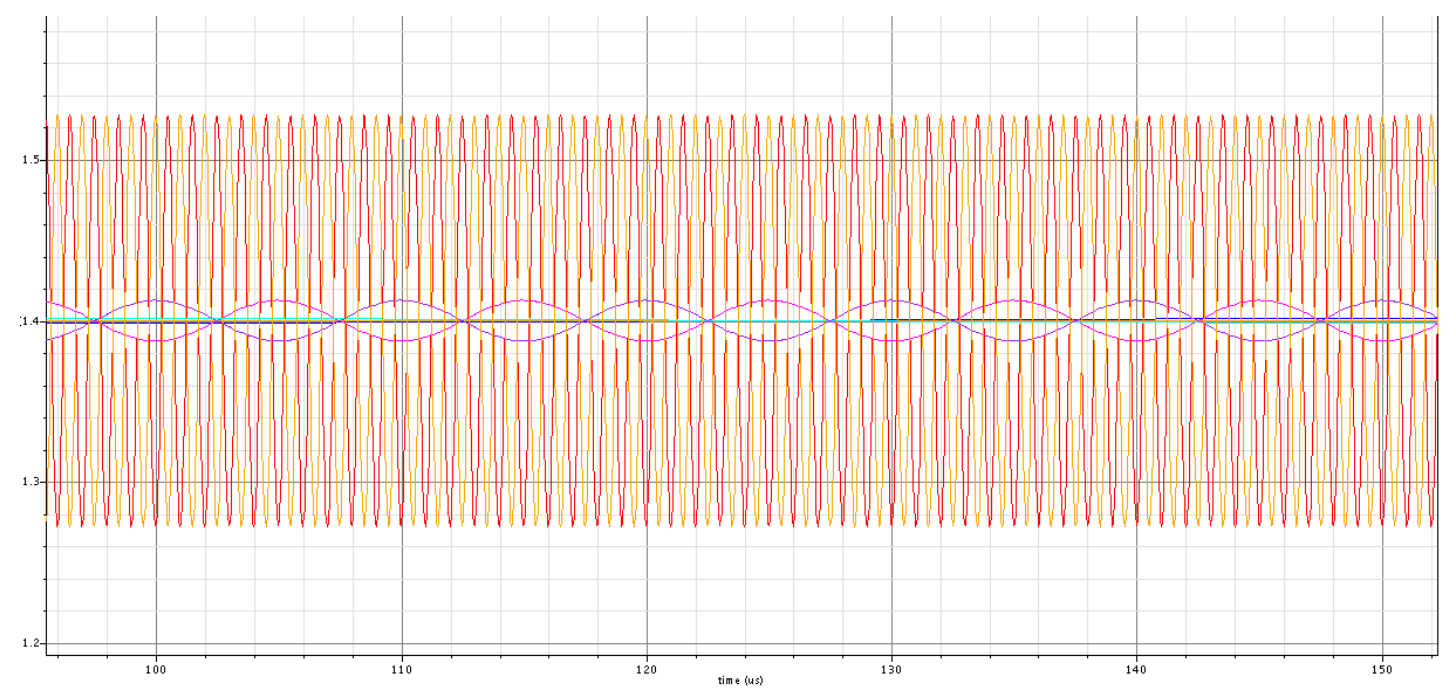

Figure 5.15: Coil's transient response

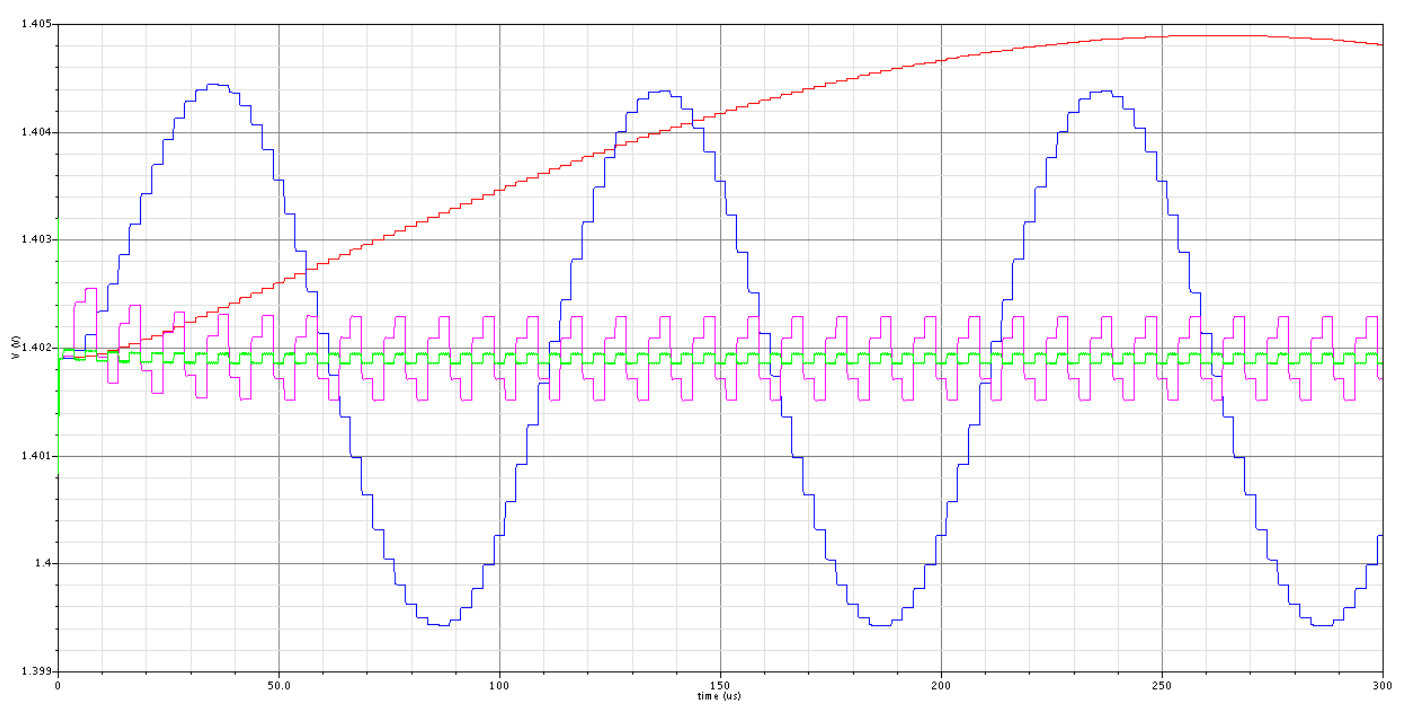

Figure 5.16: Hall sensor's transient response 


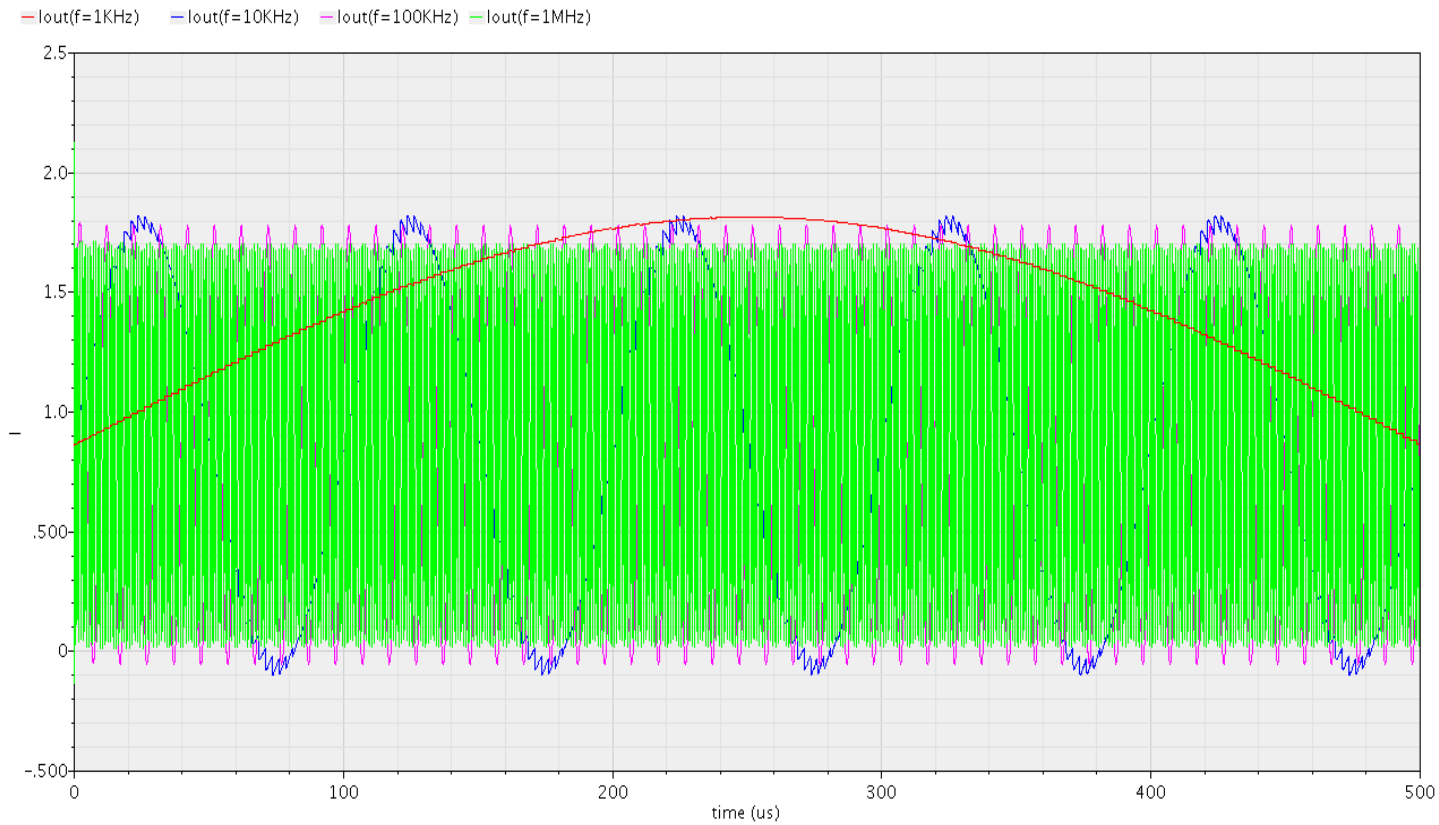

Figure 5.17: Output Current transient response

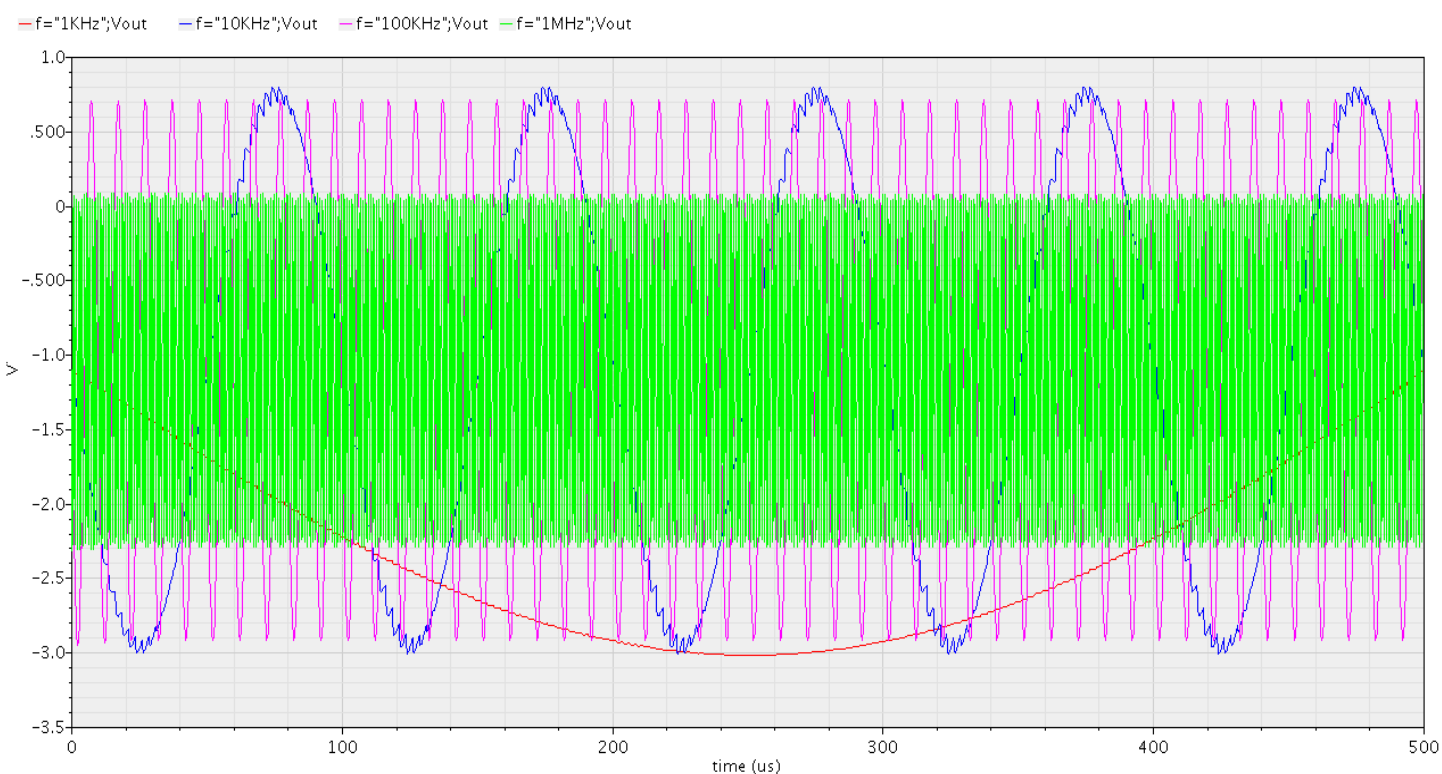

Figure 5.18: Output Voltage transient response

It should be highlighted here that the simulations with the Hall sensor at the second input lasted a period of some weeks. This is so because of the fact that the Hall sensor has digital components and thus there was not possible to have frequency response (ac) simulations. In order to get the required output response, the Hall sensor's parameters were set (with the help and guidance of the Hall sensor's designer) and also the parameters of the readout circuit. Parametric transient response simulations were done for different parameter values in order to choose the ones that would result to the same current amplitude and voltage 
Galvanically Isolated, Wide-Band Current Sensors

difference output response for frequencies lower than $1 \mathrm{MHz}$, as it is presented in Figures 5.17 and 5.18 .

As it will be described later, according to the fabricated chips measurements the results have 0 Volts $\mathrm{CM}$ at the output when the Hall sensor is connected. The setup and results of Figures 5.17 and 5.18 have been chosen in order to present the correct output functionality of the circuit having in mind that ac response simulations where not possible at this point.

\subsection{Fabricated Chips}

This work includes the fabrication and measurement of two tapeouts and also the final PCB design and measurement.

The first tape-out was sent in May 2009 and the fabricated chip was delivered in August 2009. The top-level Layout view of the first fabricated chip with pins is presented in Figure 5.19. The dimensions of this layout, including pads, are $1.950 \times 1.810 \mathrm{um}^{2}$. The microphotograph of the resulting silicon die is presented in Figure 5.20. The chips have been produced using the Multi Project Wafer service of AMS. The figure of the first fabricated chip is presented in Figure 5.21. In Figure 5.22 there is a zoomed-in view regarding the pins.

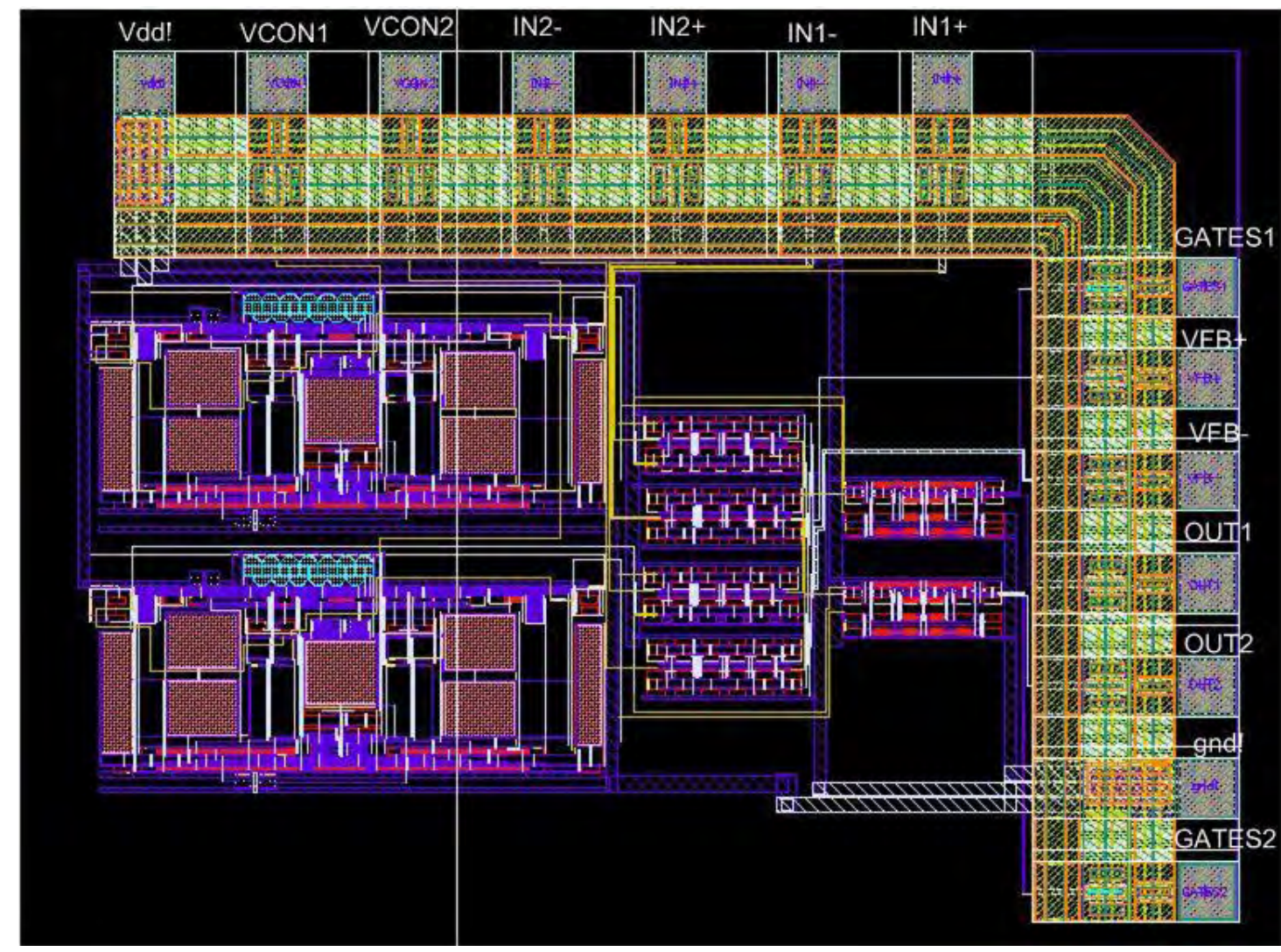

Figure 5.19: Layout view 
Galvanically Isolated, Wide-Band Current Sensors

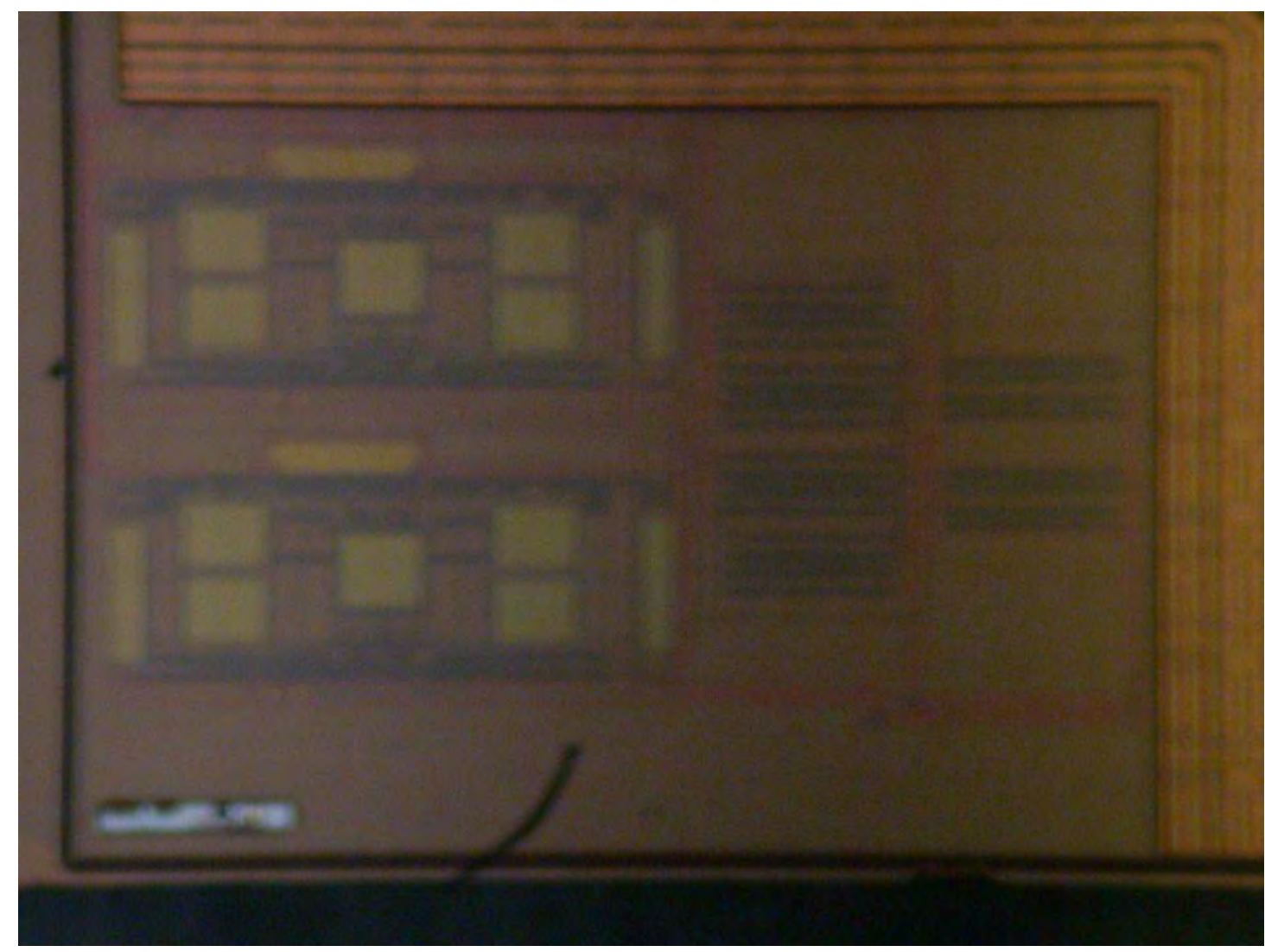

Figure 5.20: Microphotograph

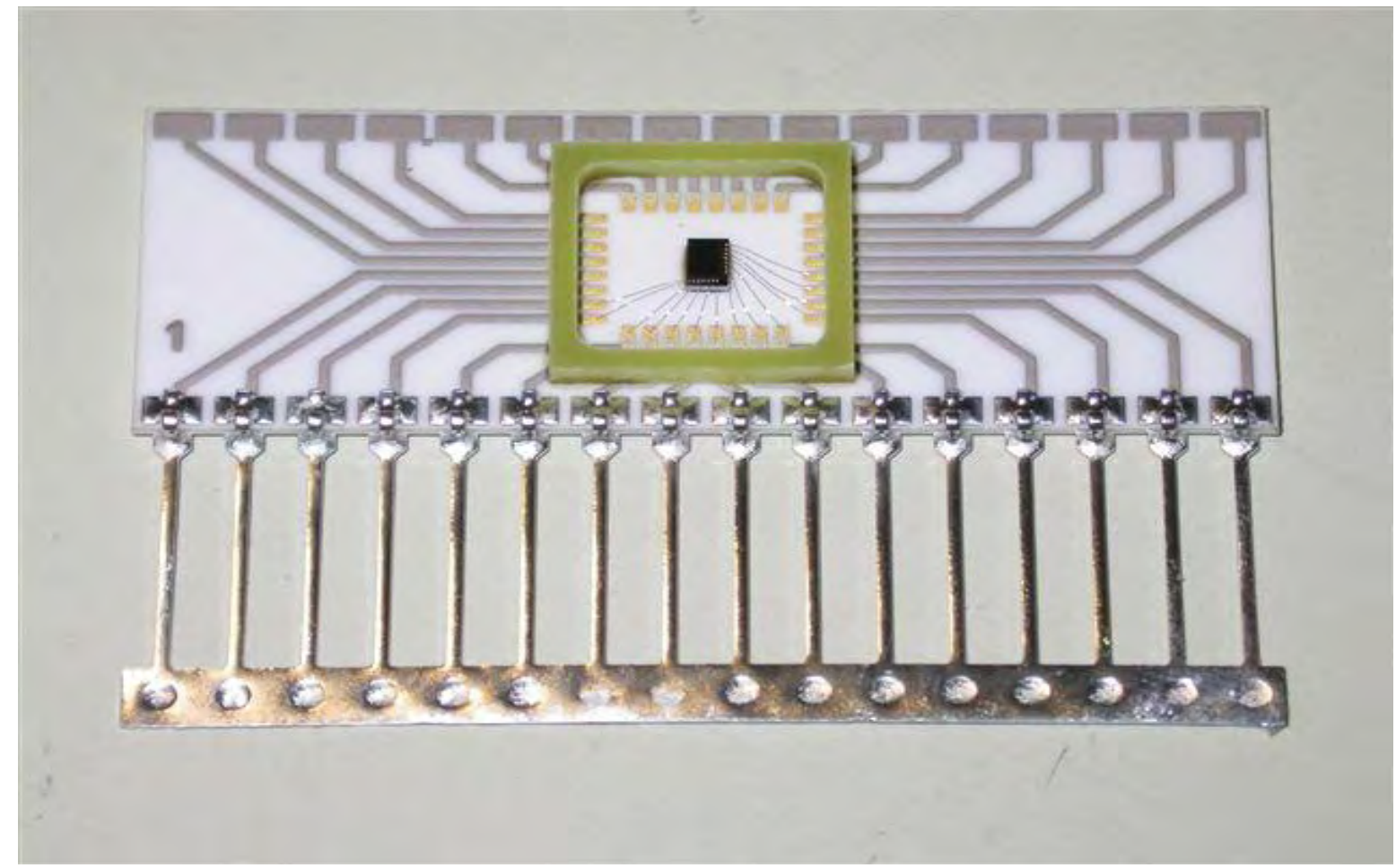

Figure 5.21: First Fabricated chip 


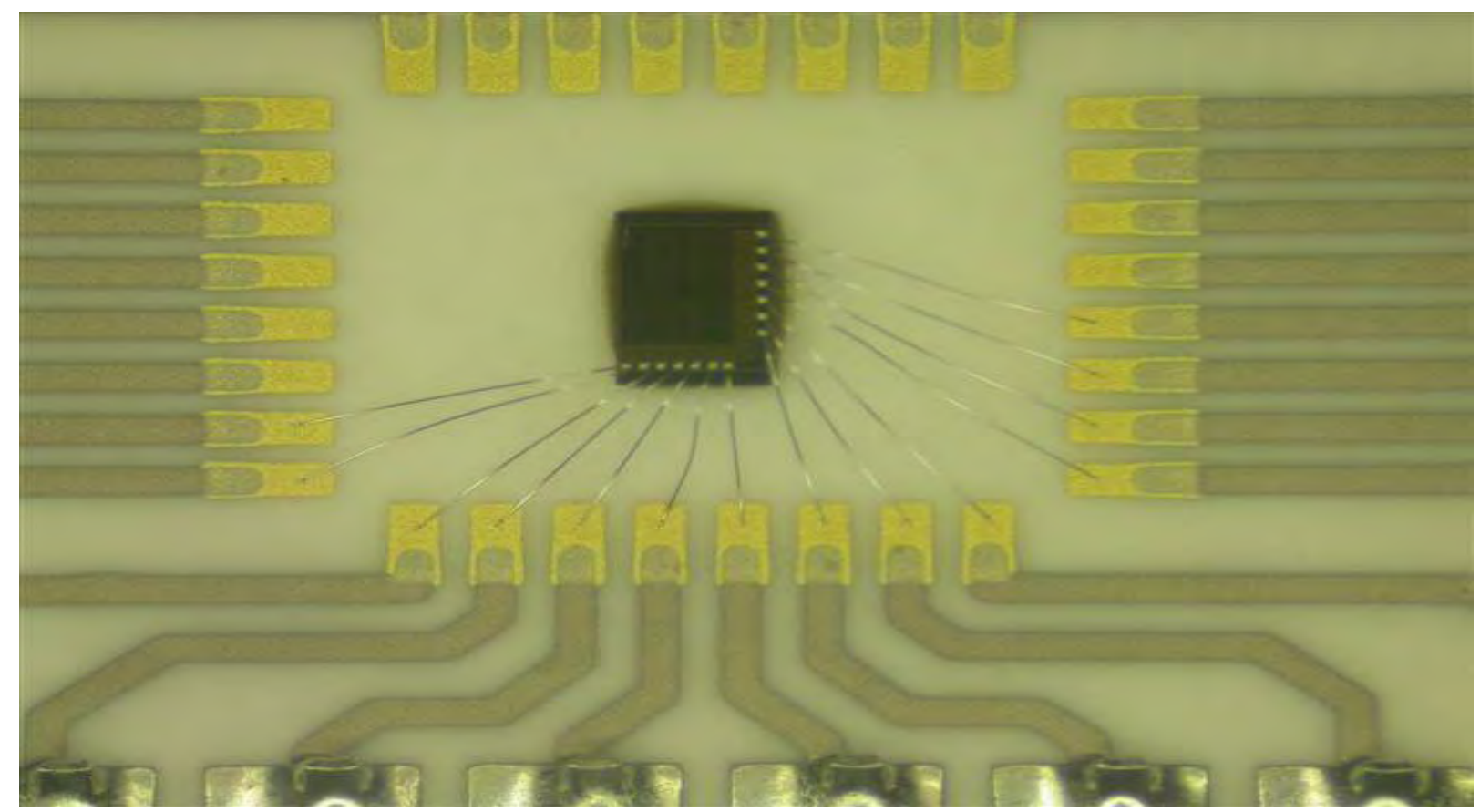

Figure 5.22: First Fabricated chip, pins

According to the results of the measurements of the first fabricated chip, improvements have been done to the design and a second tape-out was sent in February 2010. The top-level Layout view of the second fabricated chip with pins is presented in Figure 5.23. The dimensions of this layout with pads are $2.303 \times 1.810 \mathrm{um}^{2}$. The second fabricated chip was bonded on a DILL support and encapsulated and was delivered in September 2010. In Figure 5.24 , the photo of the second fabricated chip is presented. 


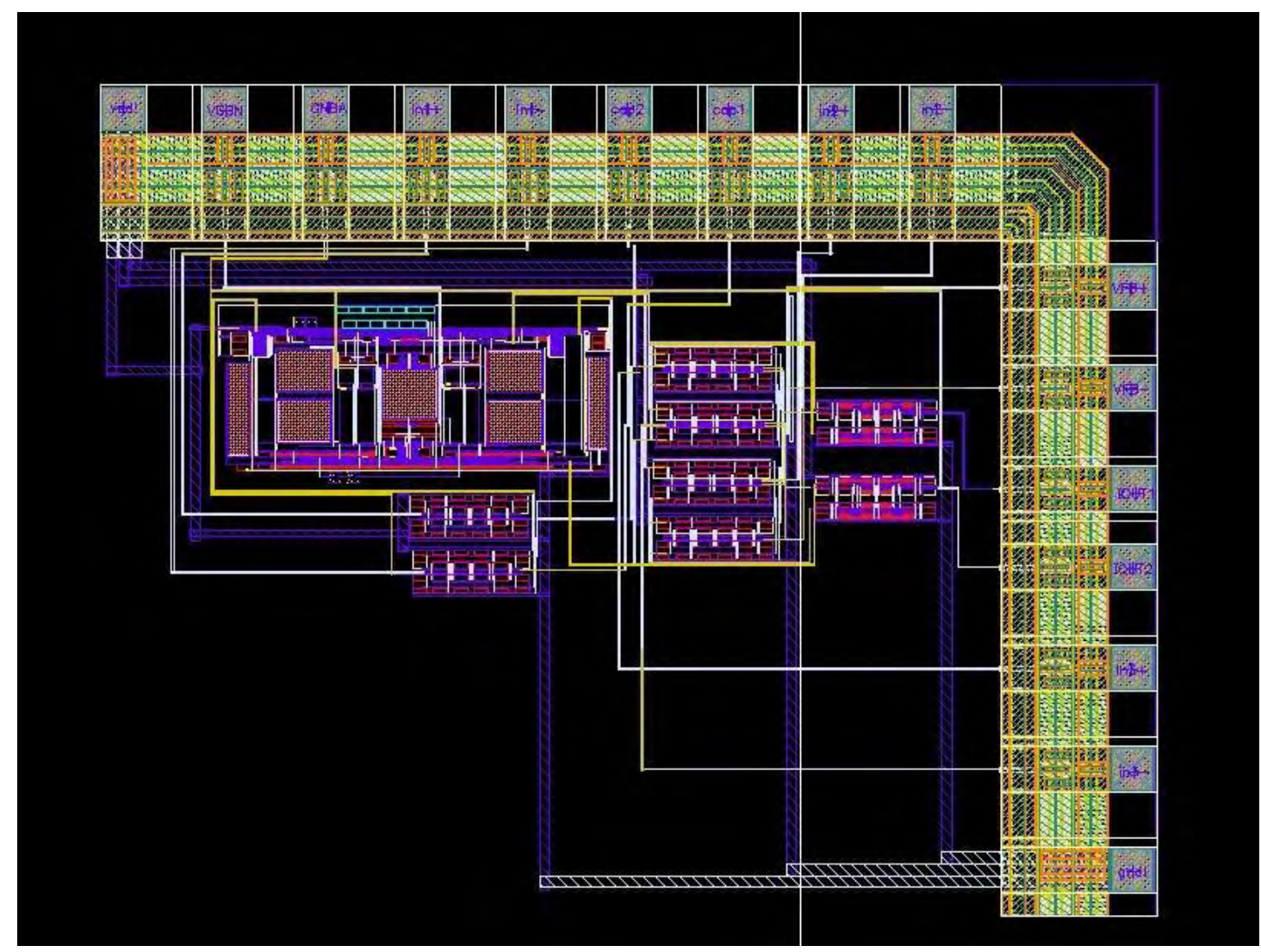

Figure 5.23: Layout view

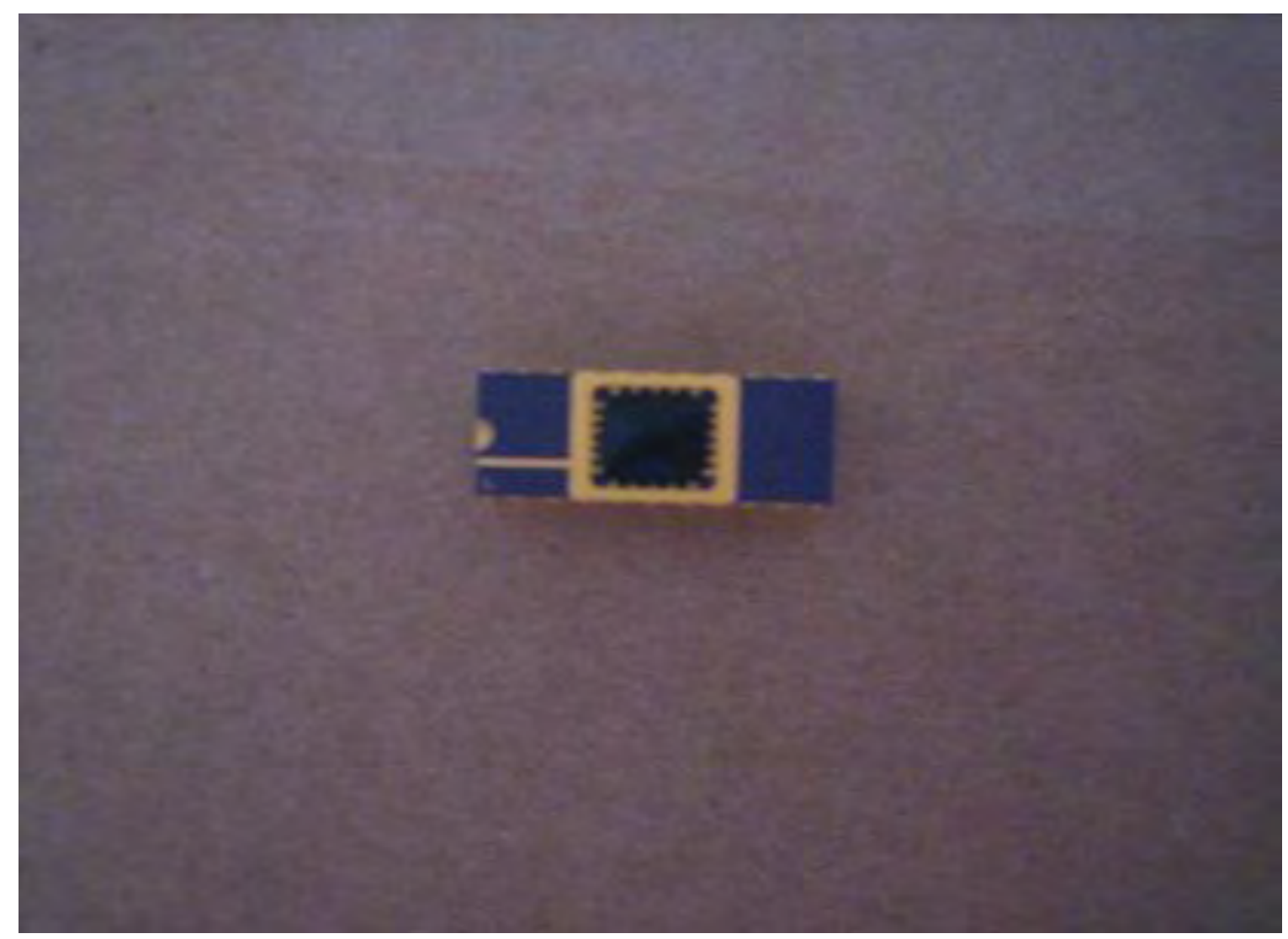

Figure 5.24: Second Fabricated chip (DILL) 


\subsection{Measurement Results}

\subsubsection{First Fabricated Chip measurements}

The first fabricated chip was different from the design of the DILL that is described in Figure 4.10. It turned out, according to the measurements of the first chip that this design architecture does not function as expected. The first design did not give the opportunity of tuning amplitude for the coil response. The measuring board of the first fabricated chip is presented in Figure 5.25.

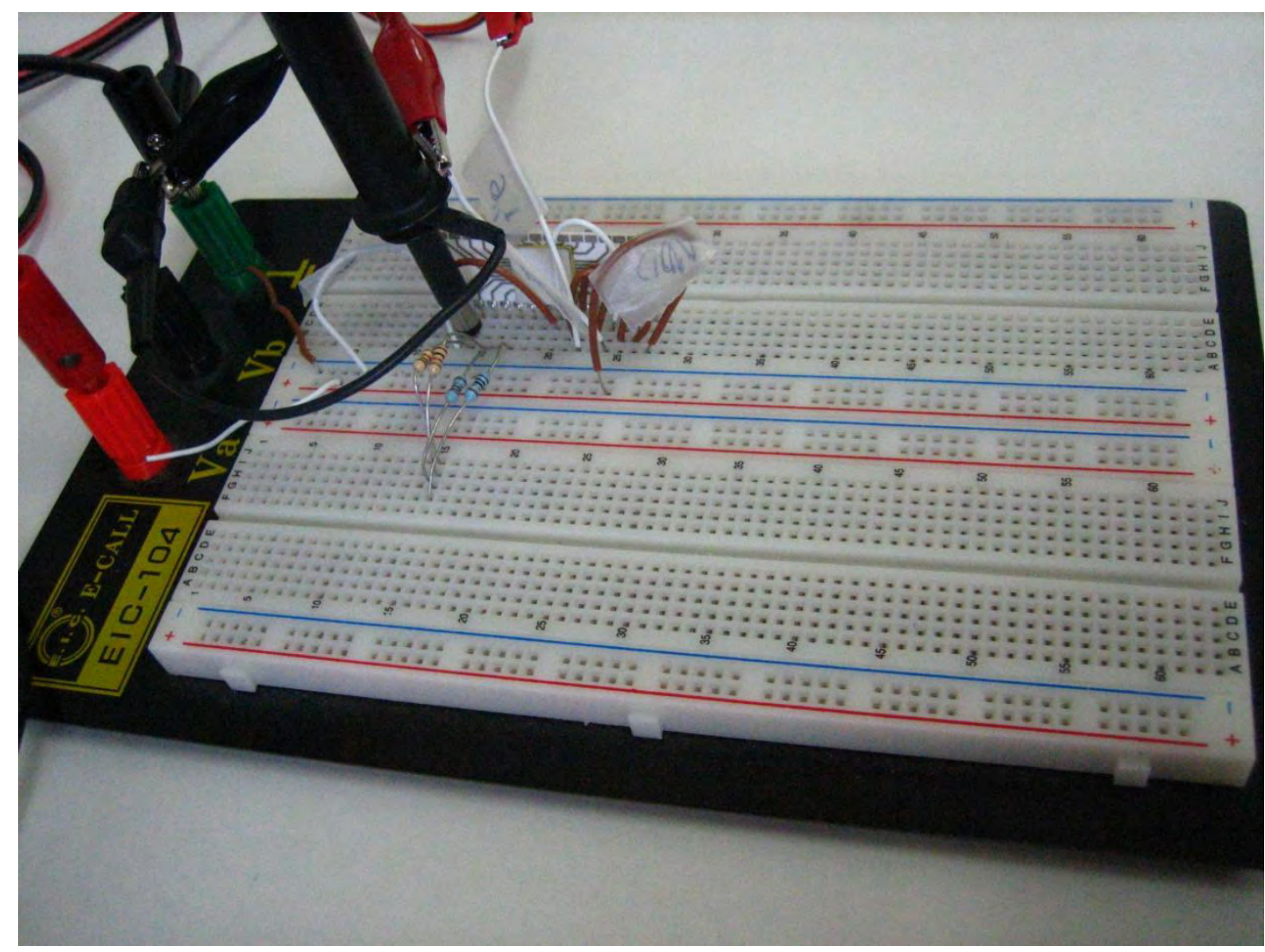

Figure 5.25: Measurement Board of the first fabricated chip

The feedback and the output resistors can be seen by inspection of Figure 5.25. The values of these resistors that are used for the measurement results are, $R_{F A}=R_{F B}=10 \mathrm{Ohm}$ (the blue ones) and $R_{01}=R_{02}=1 \mathrm{KOhm}$ (the brown ones). The measurement results are presented in the plot of Figure 5.26. The values of the input signals are $v_{\text {in2 }}=80 \mathrm{mV}(p-p)$ and $v_{\text {in } 1+}=80 \mathrm{mV}(p$ $p)$. The supply voltage is equal to $V_{D D}=3.2 \mathrm{~V}$ and $V_{C M}=1.6 \mathrm{~V}$. The output response is measured for frequency range $100 \mathrm{~Hz}-1 \mathrm{MHz}$. 
Galvanically Isolated, Wide-Band Current Sensors

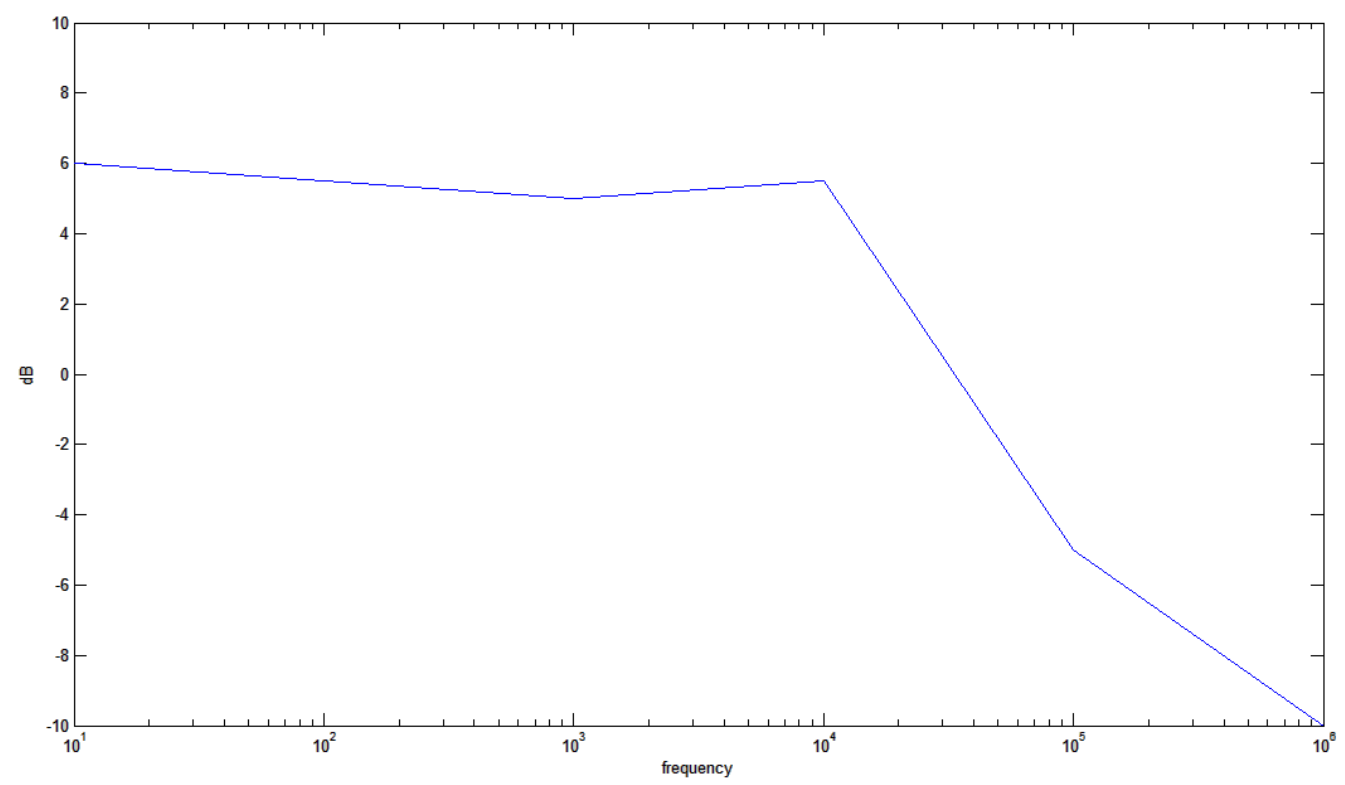

Figure 5.26: Measurement Results of the first fabricated chip

According to the above measurement results and conclusions regarding these results, there was a second design. The schematic of the second design is presented in Figure 4.10 and the function that describes the output of the circuit is presented in accordance in Chapter 4. It turned out that the gain should be improved and the functionality of the varying pole position, that has already being described, should be added.

\subsubsection{DILL Measurement Results}

The measuring board of the DILL is presented in Figure 5.27. 


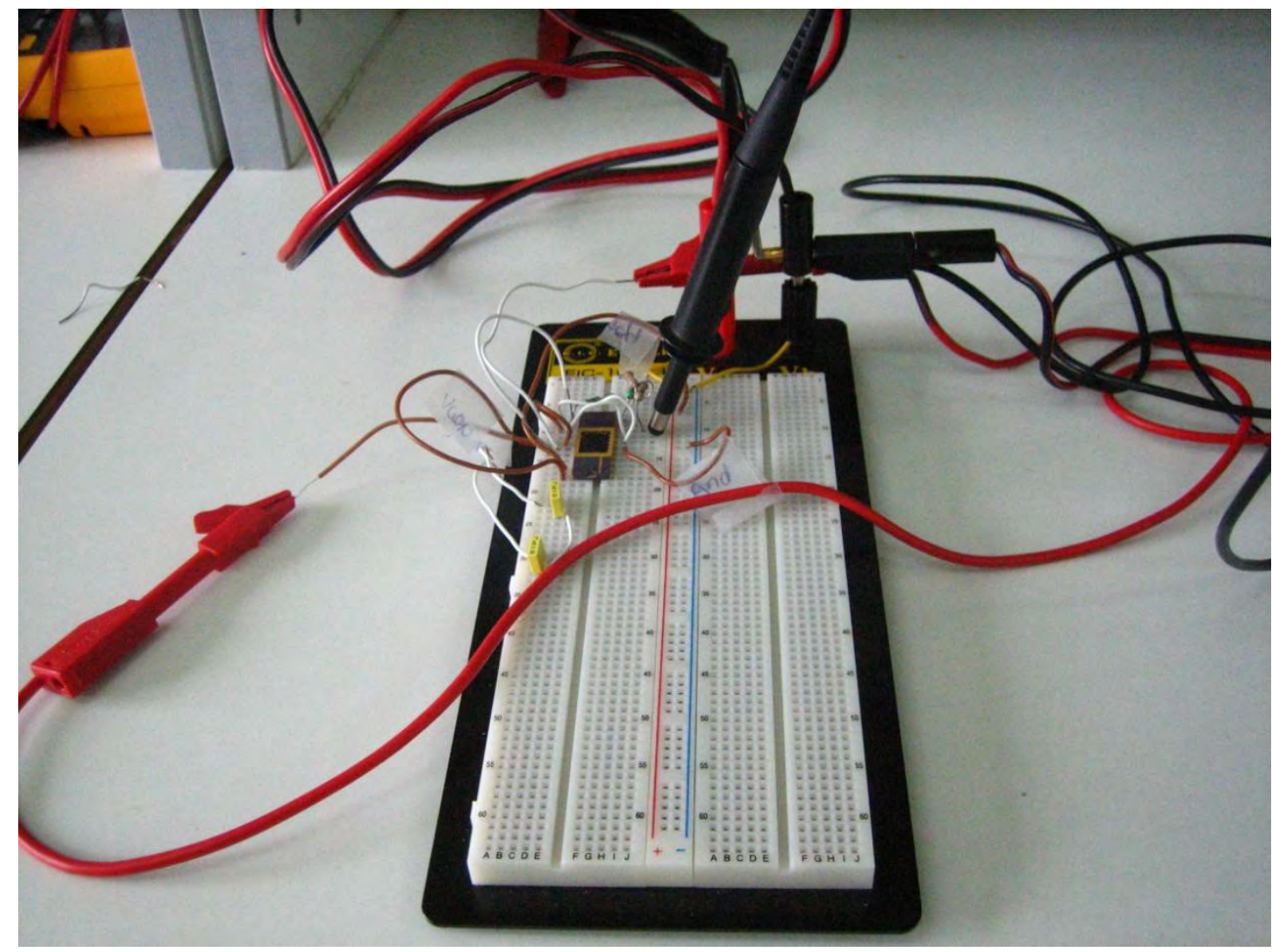

Figure 5.27: Measurement Board

From inspection of Figure 5.27, the capacitors which are called cap at the system design figure/description are connected externally. Also, from inspection of Figure 5.27 the output resistors can be seen.

In order to measure the chip, a coil is used as the first input and an RC filter is used as the second input in order to model the Hall sensor's output. The measurement results are presented in Figure 5.28. The parameters' values that are used for that measurement are, $\mathrm{R}_{\mathrm{FA}}=\mathrm{R}_{\mathrm{FB}}=1 \mathrm{Ohm}, \mathrm{R}_{01}=\mathrm{R}_{02}=1 \mathrm{~K}$ and cap $=2 \mathrm{nF}$. The supply voltage is equal to $\mathrm{V}_{\mathrm{DD}}=3.2 \mathrm{~V}$ and $\mathrm{V}_{\mathrm{CM}}=1.6 \mathrm{~V}$. 
Galvanically Isolated, Wide-Band Current Sensors

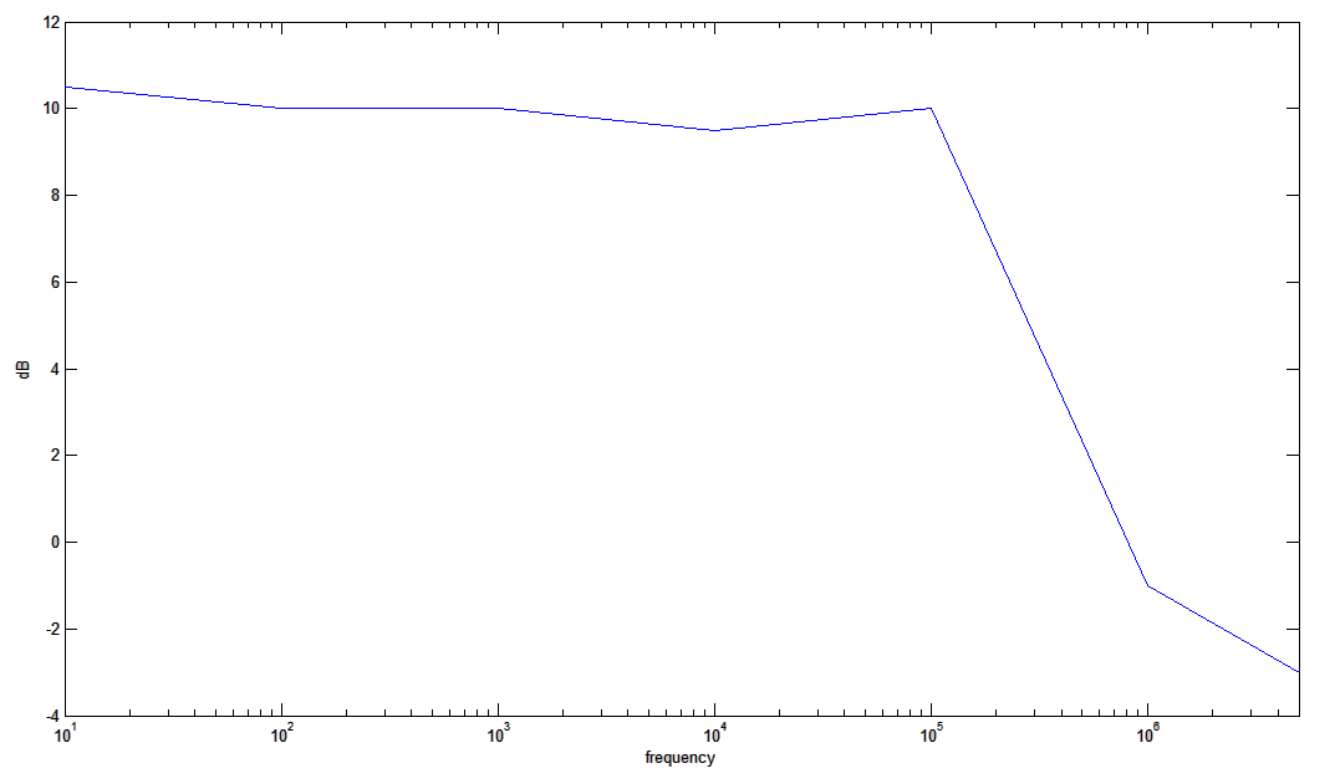

Figure 5.28: Measurement Results of the second fabricated chip

\subsubsection{PCB Measurement Results}

A Printed Circuit Board is designed, including the merging circuit (readout circuit) that is presented here and the flat coil. The schematic view of the PCB design is presented in Figure 5.29 .

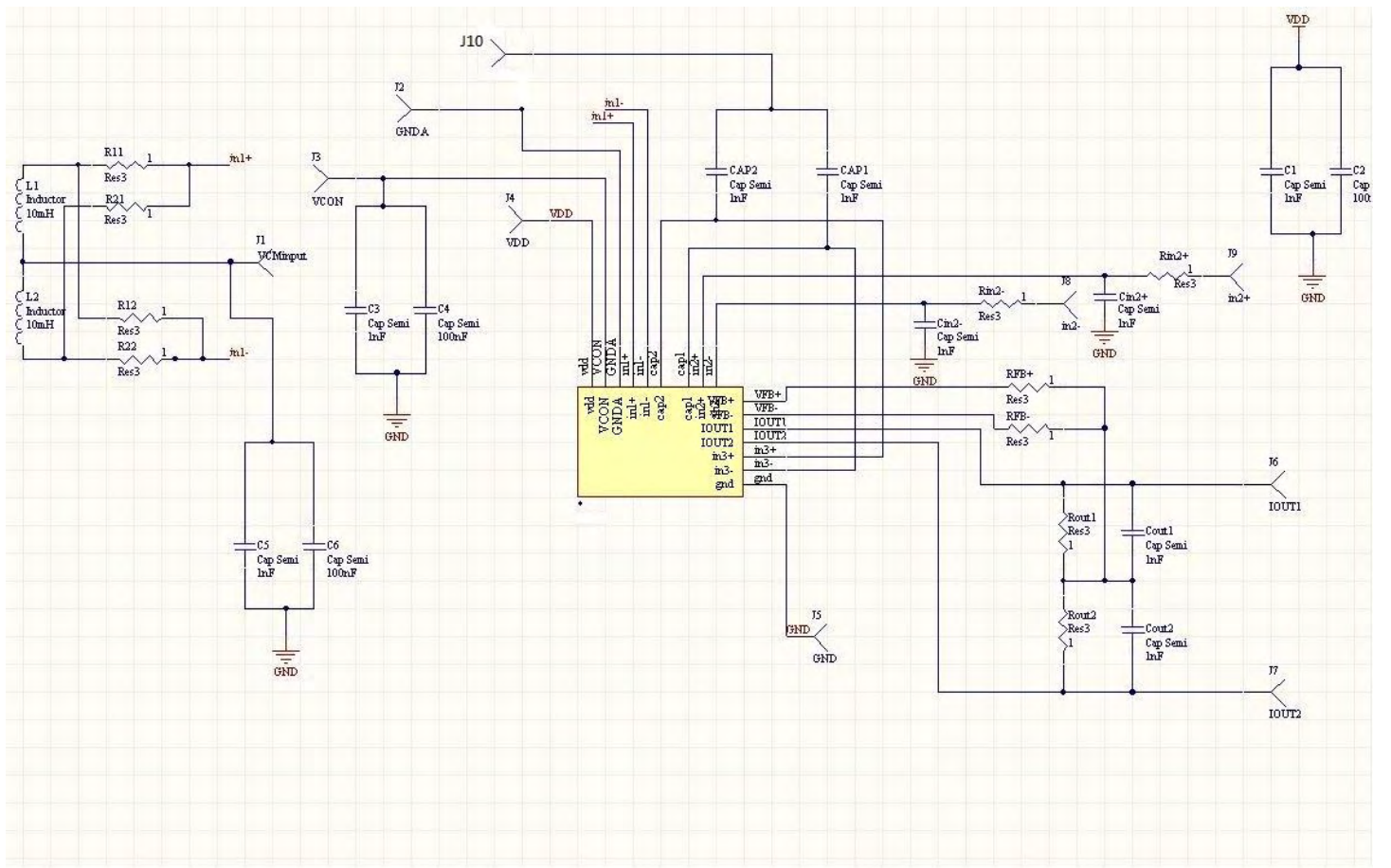

Figure 5.29: РCB schematic view 
Galvanically Isolated, Wide-Band Current Sensors

The PCB is presented in Figure 5.30

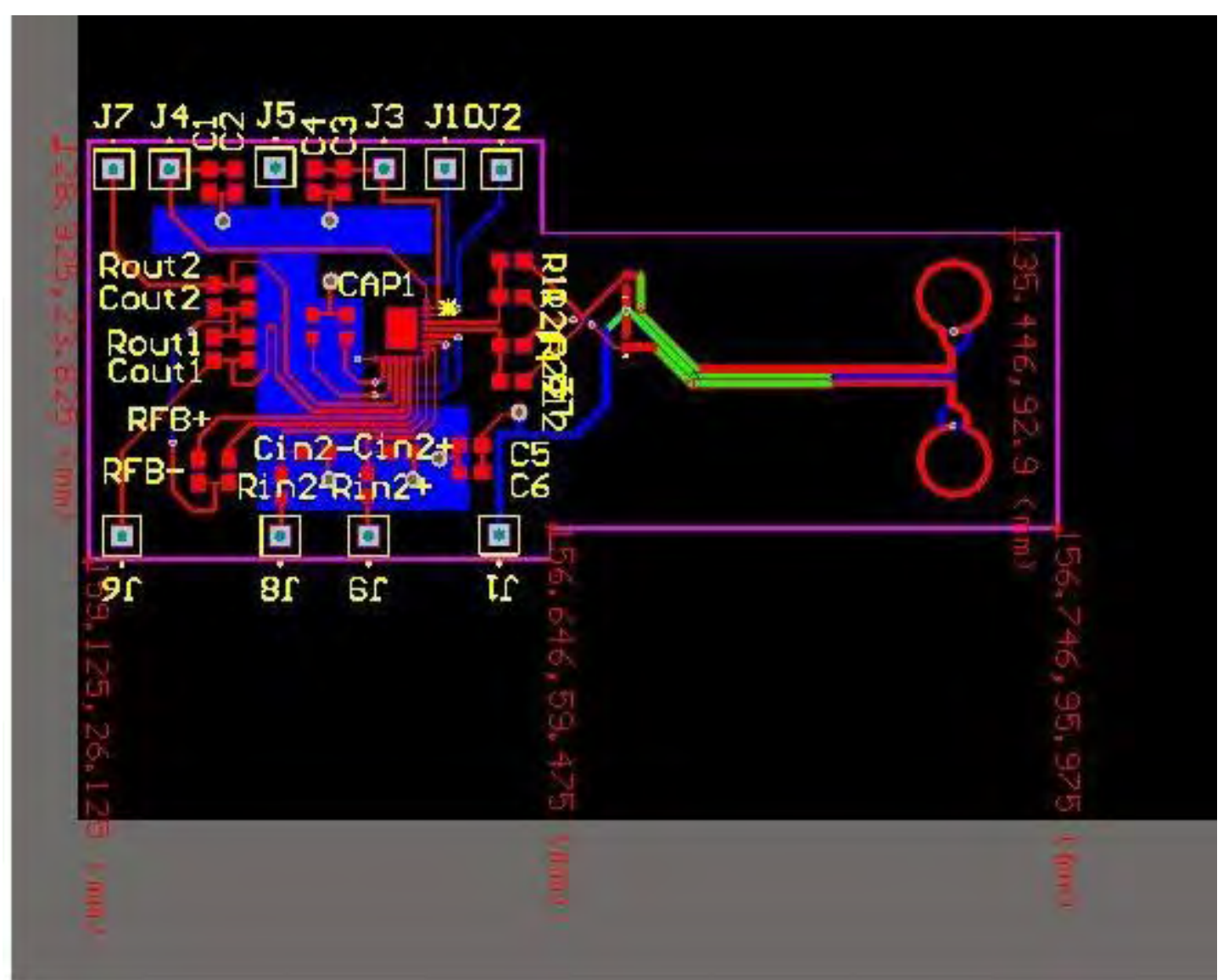

Figure 5.30: PCB

The measurement setup of the PCB, only with the coil in the one input of the merging circuit is presented in Figure 5.31. 


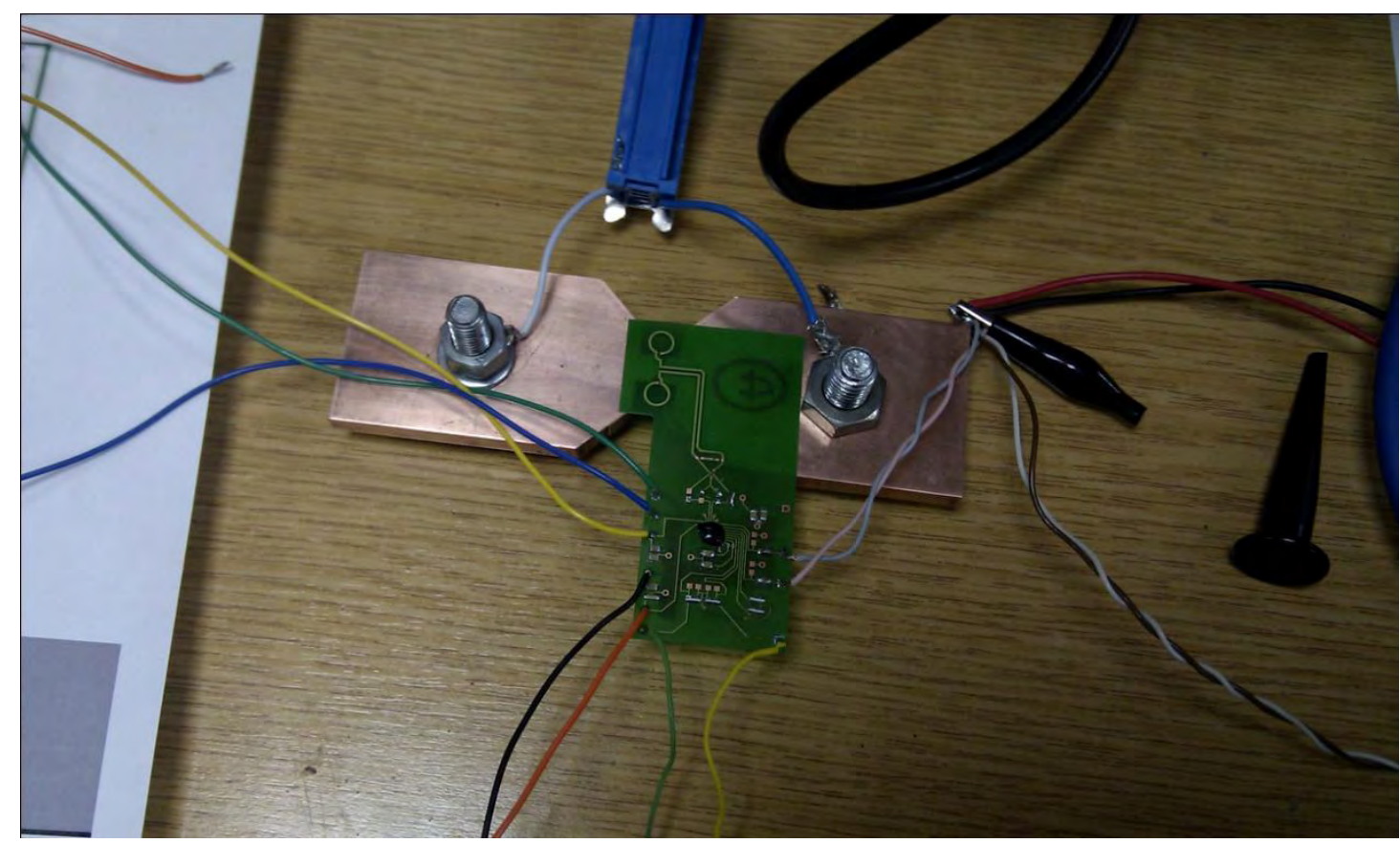

Figure 5.31: PCB measurement setup

The measurement results of the PCB with the flat coil at the first input are presented in Figure 5.31. The parameters' values that are used for that measurement are, $R_{F A}=R_{F B}=10 \mathrm{Ohm}$, $\mathrm{R}_{01}=\mathrm{R}_{02}=1 \mathrm{~K}$ and cap $=2.2 n F$. The supply voltage is equal to $\mathrm{V}_{D D}=3.2 \mathrm{~V}$ and $\mathrm{V}_{C M}=1.6 \mathrm{~V}$.

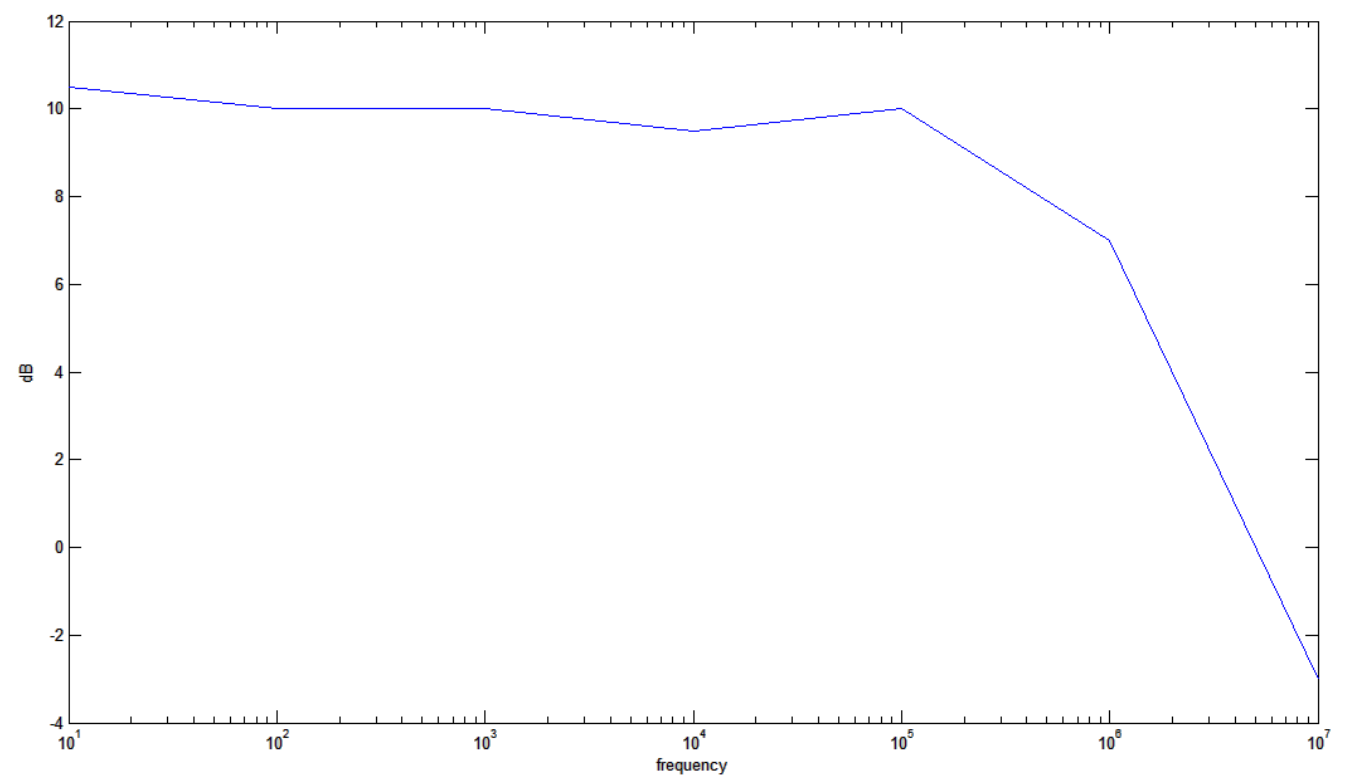

Figure 5.32: PCB measurement results, with flat coil at the first input

The measurement setup of the PCB with the flat coil connected to one input and the magnetometer connected to the other input of the merging circuit is presented in Figures $5.33 \mathrm{a}, 5.33 \mathrm{~b}$ and $5.33 \mathrm{c}$. As it can be seen by inspection of Figures $5.33 \mathrm{a}, \mathrm{b}, \mathrm{c}$, the Hall 
Galvanically Isolated, Wide-Band Current Sensors

magnetic sensor incorporates a shielding device. This device was chosen and designed according to the results of the Magnetic Field Simulator that is presented in Chapter 6 .

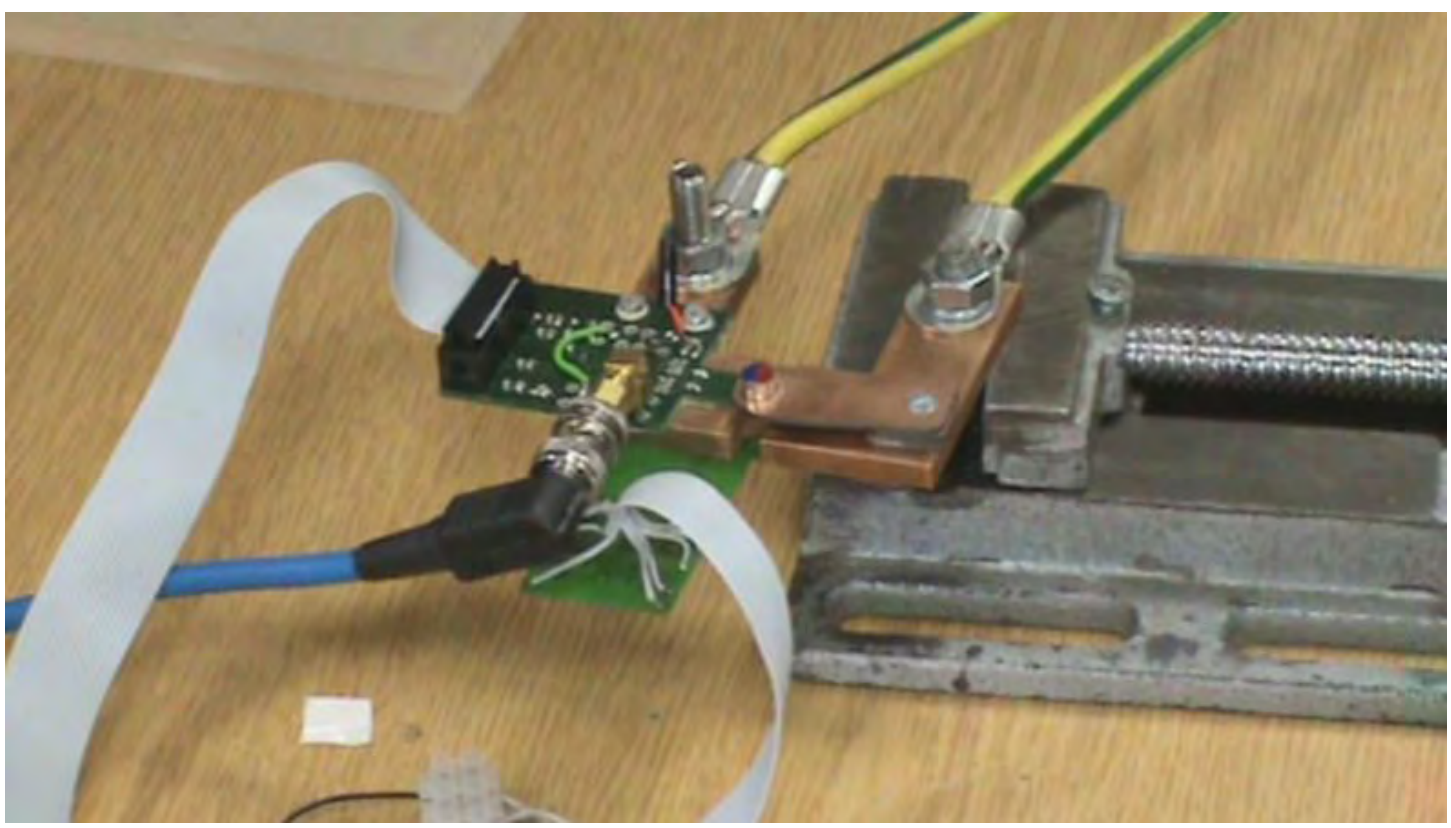

Figure 5.33a: Measurement setup

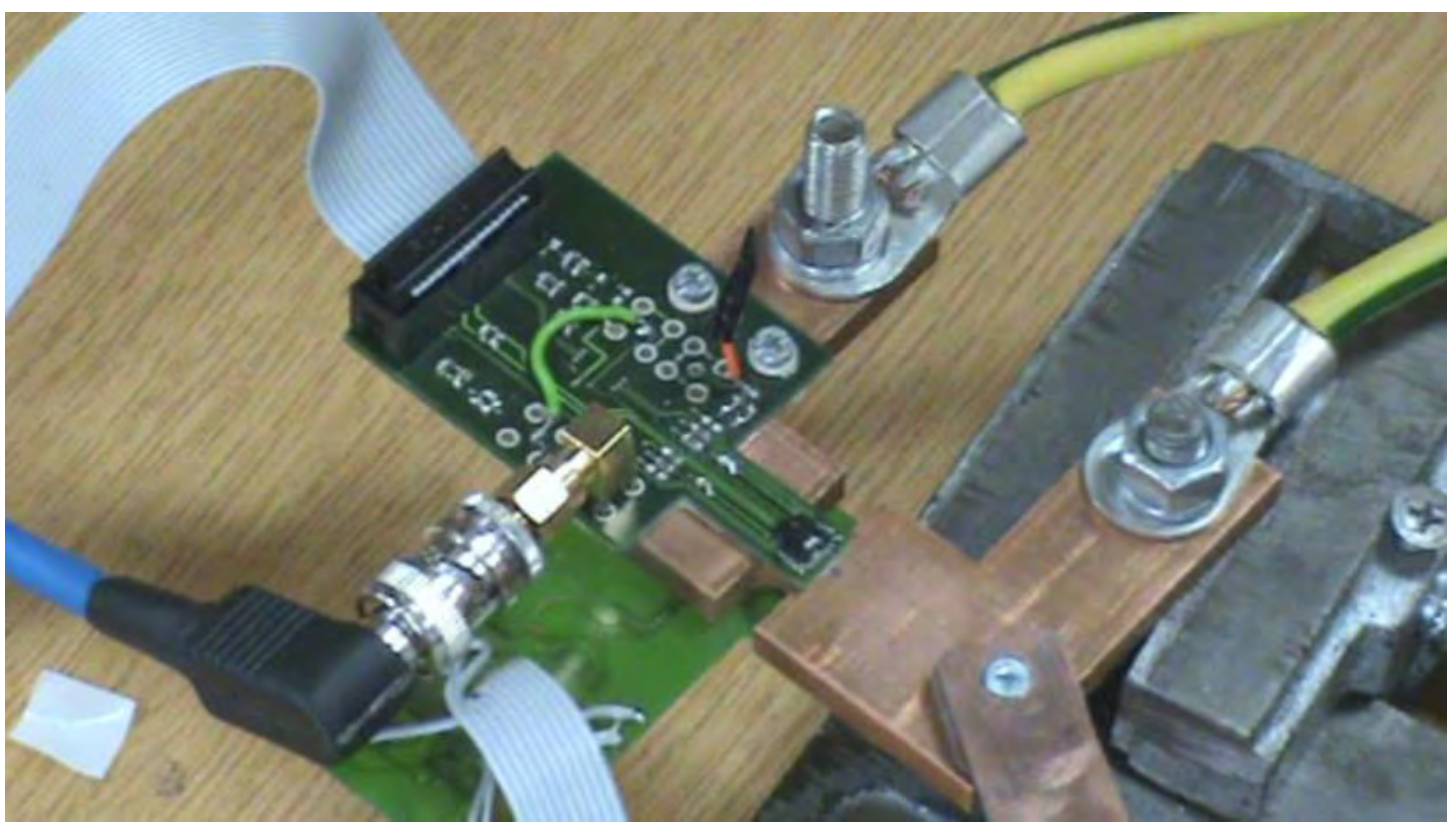

Figure 5.33b: Measurement setup 


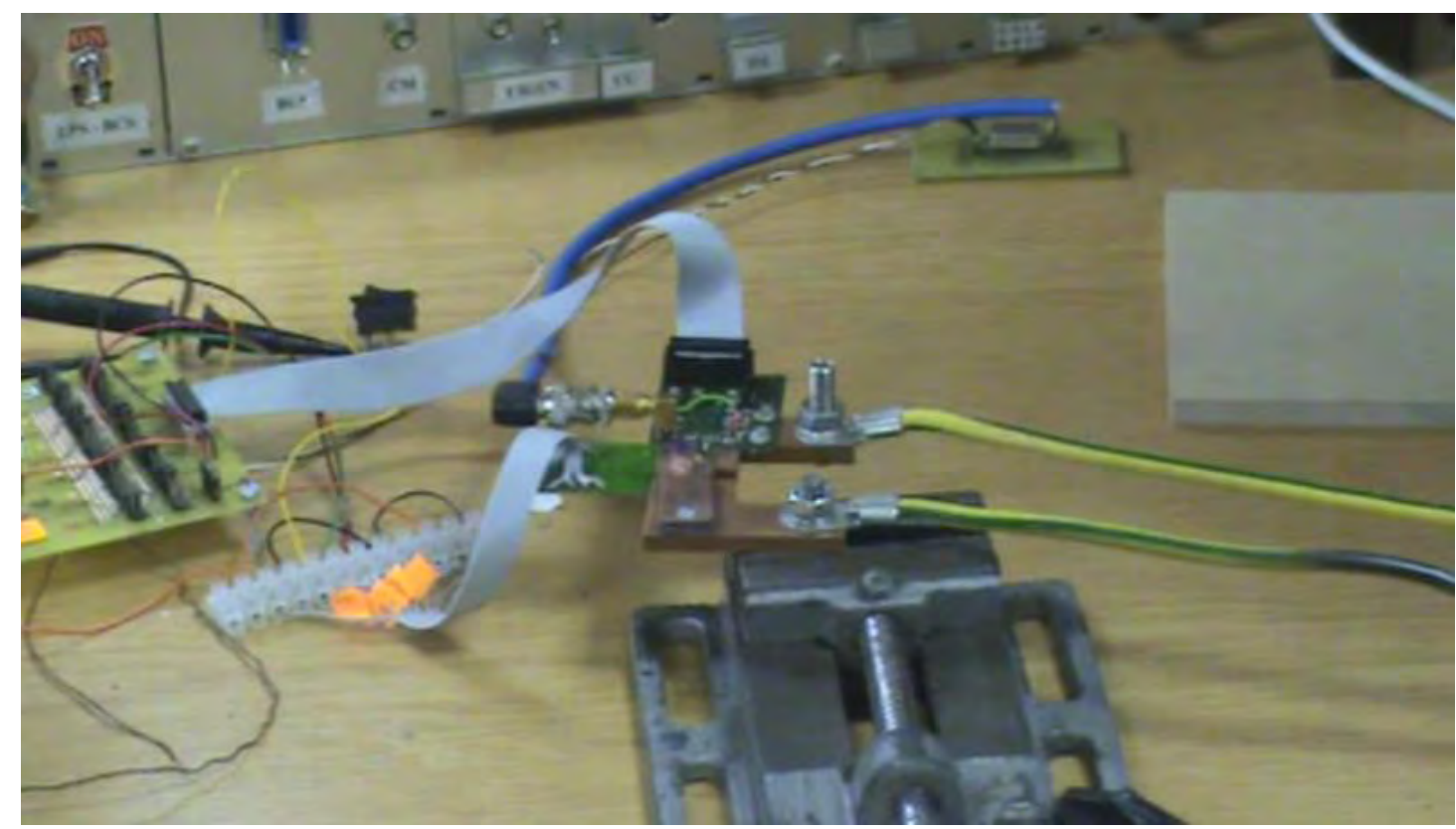

Figure 5.33c: Measurement setup

The measurement results of the whole system, with the flat pick-up coil at the first input and the magnetometer at the second input of the readout circuit are presented in Figure 5.34. The parameters' values that are used for that measurement are, $\mathrm{R}_{\mathrm{FA}}=\mathrm{R}_{\mathrm{FB}}=10 \mathrm{Ohm}, \mathrm{R}_{01}=\mathrm{R}_{02}=1 \mathrm{~K}$ and cap $=4 n F$. The supply voltage is equal to $V_{D D}=3.2 \mathrm{~V}$ and $V_{C M}=1.4 \mathrm{~V}$. The coil's signal amplitude is equal to $5 \mathrm{mV} p$-p and the Hall sensor's signal amplitude is equal to $84 \mathrm{mV} p-p$.

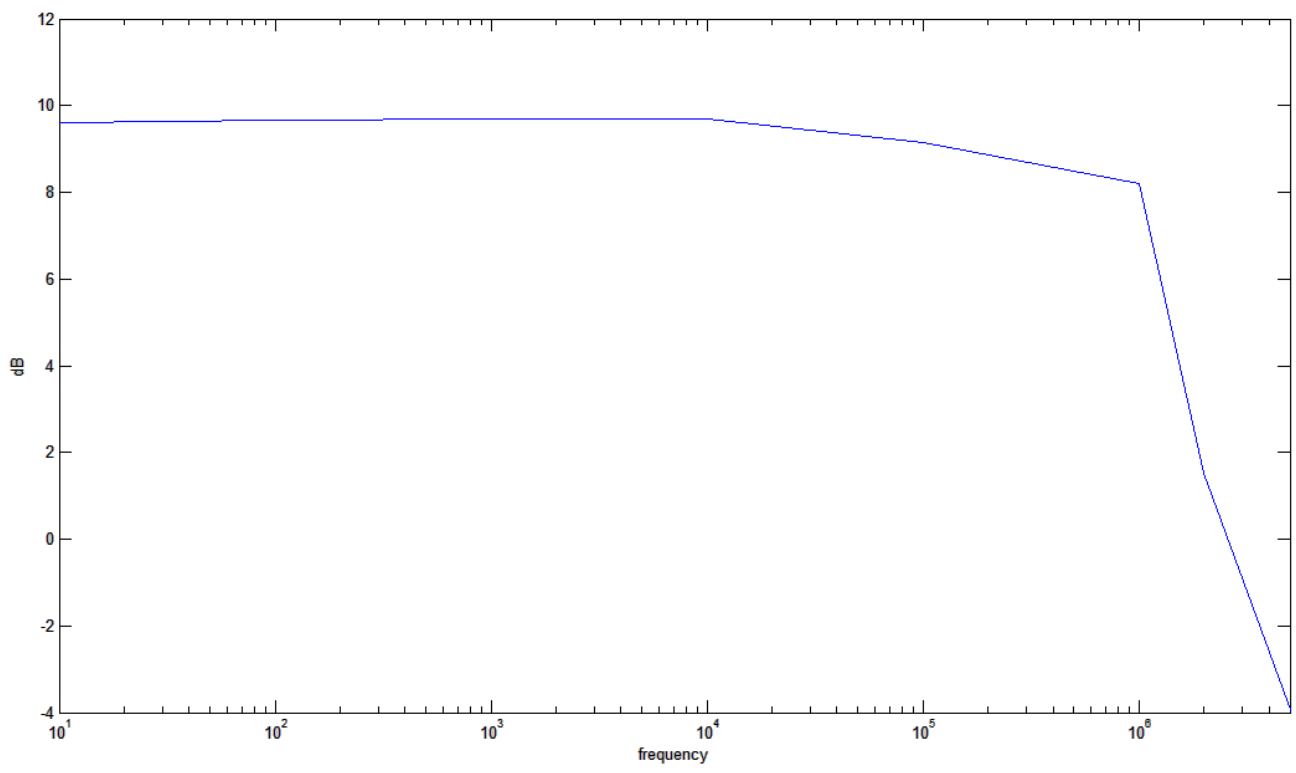

Figure 5.34: Measurement Results 
Galvanically Isolated, Wide-Band Current Sensors

\section{References}

[1] http://www.cadence.com/us/pages/default.aspx

[2] http://www.austriamicrosystems.com/

[3] Flat coil, Marjan Blagojevic, SENTRONIS a.d. Nis

[4] M.Banjevic, High Bandwidth CMOS Magnetic Sensors Based on the Miniaturized Circular Vertical Hall Device, PHD thesis.

[5] M.Banjevic, F.Liakou, B.Furrer, S.Dimitrijevic, M.Blagojevic, P.D.Dimitropoulos and R.S.Popovic, Open-loop CMOS Current Transducer with Low Temperature Cross-sensitivity, Eurosensors XXV, 2011 


\section{Magnetic Field Simulator}

\subsection{Introduction}

Magnetic Field Simulator (MFS) is an open source software tool developed in Matlab [1] script language that is capable of solving $2 \mathrm{D}$, quasi-static electromagnetic problems inside and around current conductors and/or conducting shields.

In a primitive implementation of a galvanically isolated current sensing structure, the current conductor curries current $I$ that induces flux density $\boldsymbol{B}$ sensed by the Hall elements, as it is presented in Figure 6.1. The operation of this device is limited up to few $\mathrm{KHz}$ of magnetic field frequency. High frequency magnetic field components induce Eddy Currents into the Hall plate [2]. These Eddy Currents disturb the uniform flow of the biasing current within the Hall plate, destructing the sensitivity of the device even as low frequencies as $D C$. This is a nonlinear effect [3], thus a linear electronic filter cannot be used to filter out high frequencies.

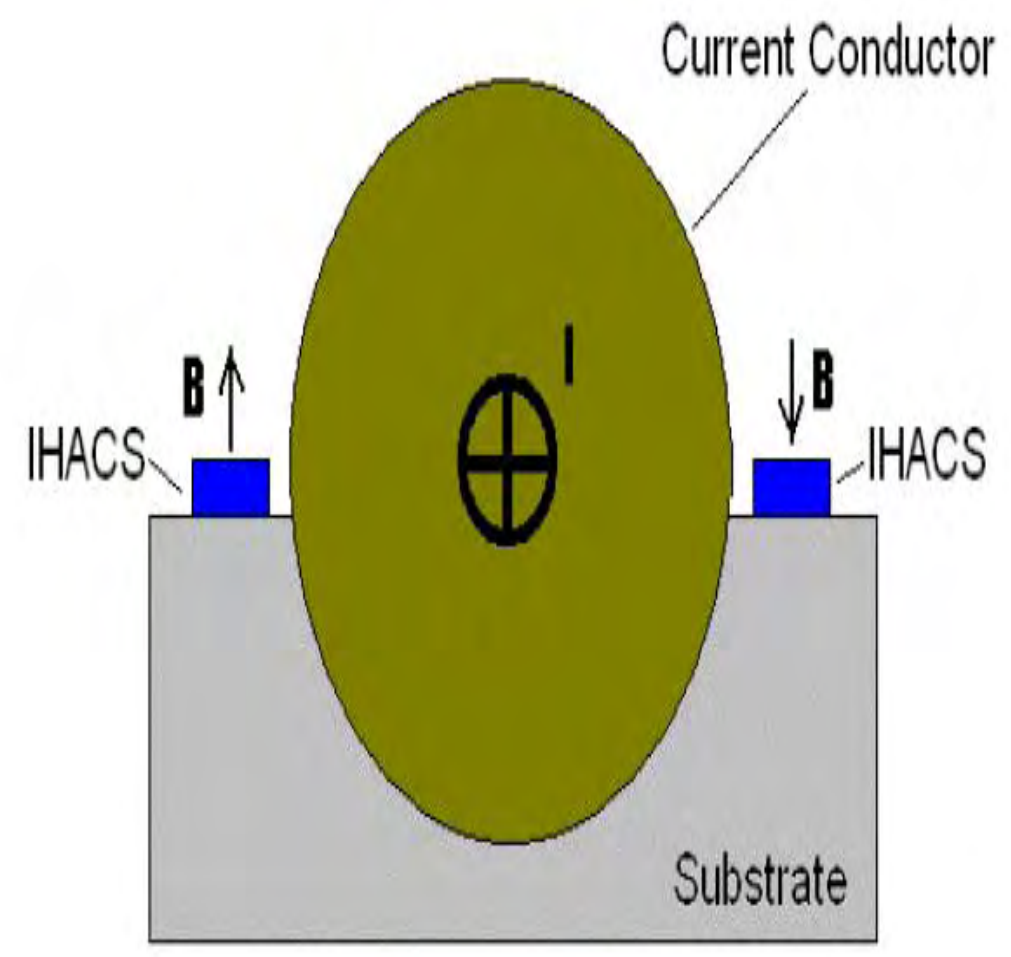

Figure 6.1: Primitive implementation of a galvanically isolated current sensing structure

The intention is the development of a flux shaping device in order to filter out high frequency (parasitic) field components. We leverage the Eddy Current effect induced by high frequency 
Galvanically Isolated, Wide-Band Current Sensors

field components [4]. A CAD tool has been developed in order to simulate the behavior of shields with different shape as the one presented in Figure 6.2.

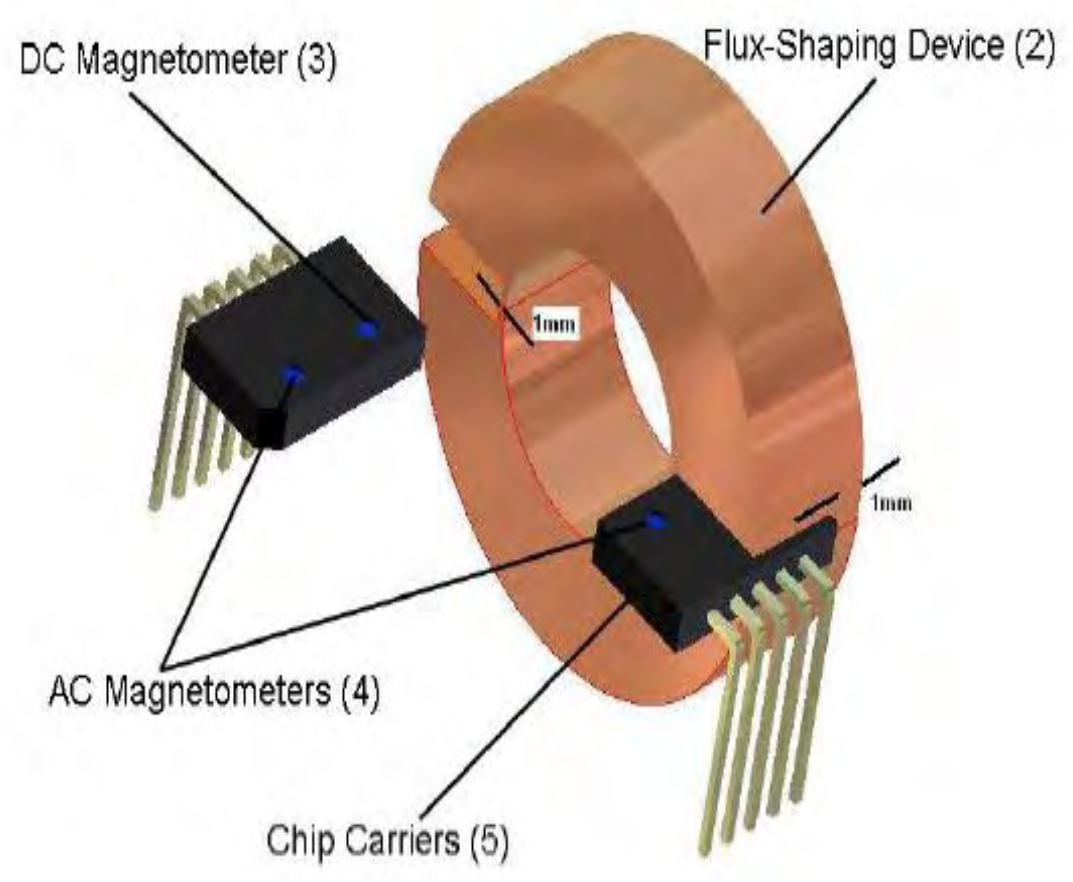

Figure 6.2: Current sensor incorporating a copper ring providing appropriate shielding against high frequency field components

\subsection{Theory}

The EM problem describing the skin effect in conductors can be formulated by means of scalar potential $\Phi$ and vector potential $\boldsymbol{A}$ that are defined in equations (6.1) and (6.2) [5], in a way to satisfy the two first Maxwell equations as presented in equations (6.3) and (6.4). Where, $\boldsymbol{\Phi}$ is the magnetic flux and $\boldsymbol{A}$ is the magnetic potential. The wave equations result from the latter two Maxwell equations as derived in equations (6.5) and (6.6). In equations (6.1)-(6.6), it is assumed that the space is linear and isotropic.

$$
\begin{aligned}
& \nabla \times \bar{A}=\mu \cdot \bar{H} \text { and } \nabla \cdot \bar{A}=-\mu \cdot \varepsilon \cdot \frac{\partial \Phi}{\partial t} \\
& \bar{E}=-\nabla \Phi-\frac{\partial \bar{A}}{\partial t}
\end{aligned}
$$


Galvanically Isolated, Wide-Band Current Sensors

$$
\begin{aligned}
& \nabla \times \bar{E}=-\nabla \times \nabla \Phi-\frac{\partial}{\partial t} \nabla \times \bar{A}=-\mu \cdot \frac{\partial \bar{H}}{\partial t} \\
& \mu \cdot \nabla \cdot \bar{H}=\nabla \bullet \nabla \times \bar{A}=0 \\
& \nabla \times \bar{H}=\nabla \times \nabla \times \frac{\bar{A}}{\mu}=\bar{J}+\varepsilon \cdot \frac{\partial \bar{E}}{\partial t} \Leftrightarrow \nabla^{2} \bar{A}-\mu \cdot \varepsilon \cdot \frac{\partial^{2} \bar{A}}{\partial t^{2}}=-\mu \cdot \bar{J} \\
& \varepsilon \cdot \nabla \cdot \bar{E}=-\varepsilon \cdot \nabla^{2} \Phi-\varepsilon \cdot \frac{\partial}{\partial t} \nabla \cdot \bar{A}=\rho \Leftrightarrow \nabla^{2} \Phi-\mu \cdot \varepsilon \cdot \frac{\partial^{2} \Phi}{\partial t^{2}}=-\frac{\rho}{\varepsilon}
\end{aligned}
$$

$\boldsymbol{H}$ is the magnetic intensity, $\boldsymbol{E}$ is the electric field, $\rho$ is the free charge density, $\varepsilon$ is the permittivity and $\sigma$ is the electrical conductivity. The current density $\boldsymbol{J}$ in a conductor is derived in equation (6.7). The continuity equation for the current is presented in (6.8). From equations (6.8) it follows that $\rho \rightarrow 0$ for $t \rightarrow+\infty$. Thus, in the steady state, the divergence of $\boldsymbol{J}$ and $\boldsymbol{E}$ are zero within the conductor, as it is presented in (6.9).

$$
\begin{aligned}
& \bar{J}=\sigma \cdot \bar{E}=-\sigma \cdot \nabla \Phi-\sigma \cdot \frac{\partial \bar{A}}{\partial t} \\
& \left\{\begin{array}{l}
\nabla \bullet \bar{J}=-\frac{\partial \rho}{\partial t} \\
\nabla \bullet \bar{J}=\frac{\sigma}{\varepsilon} \cdot \rho
\end{array}\right\} \Rightarrow \rho=\rho_{o} \cdot e^{-\sigma / \varepsilon^{-t}} \\
& \nabla \bullet \bar{J}=\nabla \bullet \bar{E}=0
\end{aligned}
$$

Following equations (6.7) and (6.8), the wave equations in the steady state become as presented in equations (6.10) and (6.11). Equations (6.10) and (6.11) can be further simplified in case propagation phenomena are neglected, as it is presented in equations (6.12), (6.13) and (6.14). In the latter case the scalar potential $\Phi$ does not vary with time and the divergence of the vector potential $\boldsymbol{A}$ is zero, as follows from equation (6.2). Equations (6.12), (6.13) and (6.14) describe generally the skin effect in current conductors.

$$
\begin{aligned}
& \nabla^{2} \bar{A}+\left(\omega^{2} \cdot \mu \cdot \varepsilon-i \cdot \omega \cdot \mu\right) \cdot \bar{A}=\mu \cdot \sigma \cdot \nabla \Phi \\
& \nabla^{2} \Phi-\omega^{2} \cdot \mu \cdot \varepsilon \cdot \Phi=0 \\
& \nabla^{2} \Phi=0 \\
& \nabla^{2} \bar{A}-i \cdot \omega \cdot \mu \cdot \bar{A}=\mu \cdot \sigma \cdot \nabla \Phi \\
& \nabla \cdot \bar{A}=0
\end{aligned}
$$


Alternatively, equations (6.15), (6.16) and (6.17) can be used to model the skin effect. These equations are derived by means of Maxwell equations (6.3) and (6.4) and - even though they assume zero divergence - they do not ensure the condition for zero divergence of $\boldsymbol{E}$ and $\boldsymbol{H}$ that is expressed by (6.4), (6.6), and (6.9). Solutions based on (6.15), (6.16) and (6.17) are correct in case of special conductor geometries - like cylindrical conductors - where the zero divergence condition is satisfied due to the physics of the problem; the solution is correct, despite the fact that zero-divergence condition is not ensured.

$$
\begin{aligned}
& \nabla^{2} \bar{H}-i \cdot \omega \cdot \mu \cdot \bar{H}=0 \\
& \nabla^{2} \bar{E}-i \cdot \omega \cdot \mu \cdot \bar{E}=0 \\
& \nabla^{2} \bar{J}-i \cdot \omega \cdot \mu \cdot \bar{J}=0 \\
& \left\{\begin{array}{c}
\hat{n} \bullet \nabla \Phi=-\frac{\hat{n} \bullet \bar{J}_{o}}{\sigma}-\frac{i \cdot \omega}{\sigma} \cdot \hat{n} \bullet \bar{A}_{o}: \text { Constant Current Excitation } \\
\Phi=\Phi_{\mathrm{o}}: \text { Constant Voltage Excitation }
\end{array}\right\} \\
& \hat{n} \bullet \nabla \Phi=-\frac{\hat{n} \bullet \bar{J}_{o}}{\sigma}-\frac{i \cdot \omega}{\sigma} \cdot \hat{n} \bullet \bar{A}_{o}=0: \text { At theConductor Side } \\
& \nabla \times \bar{B}_{o}=\bar{A}_{o}
\end{aligned}
$$

To model the skin effect and subsequently calculate the magnetic field around a current conductor, one should solve the system of elliptic equations shown in equations (6.12), (6.13) and (6.14). This imposes the need for derivation of two sets of boundary conditions, one set for $\boldsymbol{A}$ and one set for $\boldsymbol{\Phi}$. Nevertheless, the boundary condition derivation is generally a threedimensional problem. If somebody needs to reduce the problem in two dimensions the following procedure must be obeyed. The problem for $\Phi$ must be solved on a plane that is parallel to the current-flow. The aforementioned problem can be solved, provided either that the conductor is symmetric around the current-flow axis, or that the conductor has negligible or very large - dimensions along a given orientation; the conductor is two-dimensional. Then the problem for $\boldsymbol{A}$ can be solved on a plane that is normal to the current-flow axis, provided that the conductor is long enough. A convenient way to derive boundary conditions, in case of constant current excitation, is to assume a very thin current conductor, carrying the same current as the original one and being parallel with it. It can be assumed that, in a very large distance, both conductors induce the same $\boldsymbol{A}$. In the case of constant voltage excitation, the problem for $\Phi$ can be solved independently, as in the electrostatic case. Then $\boldsymbol{A}$ is calculated by means of Neumann boundary conditions that are $n \bullet \nabla \boldsymbol{A}=0$ [4]. Neumann boundary condition is a type of boundary condition, when imposed on an ordinary or a partial differential equation it specifies the values that the derivative of a solution is to take on the boundary of the domain. It is essential to notice that the normal components of $\boldsymbol{A}, \boldsymbol{J}$, and $\boldsymbol{E}$ are zero at the 
current-conductor boundary, because the current cannot leave the conductor. Boundary conditions for the aforementioned cases are presented in equations (6.18), (6.19) and (6.20).

\subsection{Magnetic Field Simulator (MFS)}

MFS is a pure 2D Partial Differential Equation problem solver. It assumes that the conductor length (z-dimension) is infinite. As a result one may easily prove that only z-components of $\boldsymbol{A}$ $=A \cdot \boldsymbol{z}, \boldsymbol{J}=\boldsymbol{J} \cdot \mathbf{z}$, and $\boldsymbol{E}=E \cdot \mathbf{z}$ exist while $\mathrm{x}$-, y-components are zero. Similarly, $\boldsymbol{B}=B_{X} \cdot \boldsymbol{X}+B_{Y} \cdot \boldsymbol{y}$. In this case equations (6.12), (6.13) and (6.14) become as it is presented in equations (6.21), (6.22) and (6.23). For constant current excitation, where the total current is denoted with $I$, equation (6.23) may also be applied. The system of equations (6.22) and (6.23) is solved iteratively.

$$
\begin{aligned}
& \left\{\begin{array}{c}
\nabla^{2} \Phi=0 \\
\widehat{n} \bullet \nabla \Phi=0
\end{array}\right\} \Rightarrow \nabla \Phi=c \\
& \nabla^{2} A-i \cdot \omega \cdot \mu \cdot A=\mu \cdot \sigma \cdot c \\
& \oint_{S} \bar{J} \bullet d \bar{S}=-\sigma \cdot \oint_{S}(c+i \cdot \omega \cdot A) \cdot d S=I
\end{aligned}
$$

The simulator algorithms that are written have been implemented in Matlab so as to form a useful software tool. This tool has been employed for the simulation and optimization of FluxShaping devices that may be optimally used to filter out high frequency (parasitic) magnetic field components in Current sensors.

\subsubsection{Simulator Description}

MFS simulator has as input domain properties and parameters values, calculates and gives as a result simulation data. These simulation data include the $z$-components of magnetic vector potential $\boldsymbol{A}$ and current density $\boldsymbol{J}$, and the $x, y$ components of magnetic flux density $\boldsymbol{B}$. These data are stored and used in order to plot the results.

Before setting-up a simulation, the geometry of the Partial Differential Equation (PDE) problem needs to be specified. That is accomplished by the specification of the Geometry Description matrix gd and the Name Space matrix ns. Each column of $g d$ matrix corresponds to an object in the solid geometry model. Four types of solid objects are supported, circle solid, polygon solid, rectangle solid and ellipse solid. Each column of $n s$ matrix contains $\mathrm{N}$ characters (one character per row) composing the name of the solid object that is described in the corresponding column of the $g d$ matrix. There are two ways to define $g d$ and $n s$ matrixes. The first one is to type them manually in Matlab workspace and the second one is to use Matlab pdetool function that launches a user friendly GUI environment. In either case gd and $n s$ matrixes are temporarily stored in Matlab Workspace as Matlab Variables and are used by MFS function makeg to generate the Decomposed Geometry matrix $g$. This matrix is 
required by MFS function which is called FEM, in order to execute the simulation. In order to execute the makeg function, a parameter that is called bdim must be specified. This parameter defines the distance measured from point $(0,0)$ at which the magnetic flux density is not affected by the special conductor shape. Parameter bdim can be calculated by using equation (6.24)

$$
B=\mu \cdot \frac{I}{2 \cdot \pi \cdot b_{\text {dim }}}
$$

Function makeg stores the Decomposed Geometry matrix $g$ in the MATLAB Workspace in MATLAB Variable format. Moreover, it saves $g$ and $g d$ matrixes in the makeg.mat and the makeg.par files respectively. A makeg.log file is also created.

Apart from defining the geometry of the 2D solids, their type must be specified with respect to the solid functionality in the PDE problem. Four kinds of types are supported, current conductor, passive shield, infinite current conductor and vacuum.

- Current conductor is a device connected to a power source, in which the current distribution is affected by eddy current effects.

- Passive shield is a conductive device that is not connected to a power source, in which the current distribution is attributed completely to eddy current effects resulting to zero total current.

- Infinite current conductor is a device connected to a power source, in which the current distribution is not affected by eddy current effect and is therefore constant.

- Vacuum, in which no current conduction is allowed.

The domain properties are defined by means of the MFS function makeg.

Also, a number of parameters must be defined as the simulator's input.

These parameters are:

- Mesh Density (meshref): Defines the density of the mesh. This parameter is a positive integer and usually takes values between 2 and 4 . Values larger than 4 may force the simulator to stall.

- Solution Tolerance (tolerance): MFS uses an iterative algorithm which finish when the absolute difference between two subsequent solutions become smaller than the tolerance parameter. This parameter is a positive double number and takes values between $10^{-2}$ and $10^{-5}$, depending on the problem.

- Max Iterations (iter): This parameter sets the amount of the algorithm's iterations. It is a positive integer and takes values between 100 and 1000 .

- Max x-coordination to export results ( $x$ max): MFS exports the solution in a way that can be used with Matlab for further analysis. The solution is saved in a rectangular space that is 
defined by the vertexes (-xmax -ymax), (xmax -ymax), (xmax ymax) and (-xmax ymax). Parameter $x$ max is positive double number.

- Max y-coordination to export results (ymax): MFS exports the solution in a way that can be used with Matlab for further analysis. The solution is saved in a rectangular space that is defined by the vertexes (-xmax -ymax), (xmax -ymax), (xmax ymax) and (-xmax ymax). Parameter ymax is positive double number.

- Export Accuracy (xydim): This parameter defines the discrete step between points in [-xmax xmax] and [-ymax ymax] intervals that are denoted as xstep and ystep respectively. It is determined by the relations $x$ step $=2 \cdot x \max / x y \operatorname{dim}$ and $y$ step $=2 \cdot y$ max $/ x y d i m$.

- Permeability $(m u)$ : This parameter defines the permeability of conductors and shields.

- Conductivity (sigm): This parameter defines the conductivity of conductors and shields.

- Conductor Current (Isource): This parameter defines the total current that flows in all conductors.

- Frequency $(f)$ : This parameter defines the frequency of Isource.

\subsubsection{Simulator Functions}

The simulator consists of six functions which are, makeg, makeb, makebscript, setfempar, FEM, pdeplot.

First of all, makeg function is set and executed. This function takes as parameters, $g d, n s$, bdim and pathdef. Parameter pathdef contains a string that specifies the path in which makeg.log, makeg.par, and makeg.mat files are saved. When this function is executed Matlab asks the user to define the domain properties. Thus, the Decomposed Geometry matrix $g$ is calculated and stored in the Matlab Workspace, in Matlab variable format. Also, the gd matrix is saved.

After that, the setfemparam function is executed. This function has as parameters, pathdef and fileid. Parameter pathdef has the same definition as for makeg function. Parameter fileid can be used to attach a string to the name of the parameter file. This functionality gives the user the opportunity to save the results of multiple simulations with different parameters (for example with different frequency) but having the same geometry. When this function is executed, Matlab asks from the user to define the algorithm's parameters, that have been described above. After that, the algorithm's parameters are stored in the Matlab Workspace, in a .par file.

Then, functions makeb and makebscript are executed. These functions are not called by the user but are called and executed internally by function FEM. FEM is the main simulator's function. FEM function takes as parameters pathdef and fileid. FEM reads the geometry data and the algorithm parameter data and calculates and stores the results in files in the Matlab Workspace. 
Galvanically Isolated, Wide-Band Current Sensors

Now that the calculations have finished, the simulator is ready to plot and present the results. There are two ways to present the results that have been calculated by FEM function. The first one is by means of standard Matlab plot function and the second one is by special Matlab pdeplot function. For the first option variables $A x y, J x y, B X x y$ and $B Y x y$ can be used, while the latter requires variables $A, J, B X$ and $B Y$ in addition to $p, e$ and $t$. The syntax of both functions is described in Matlab Help.

\subsection{MFS Simulation Results}

MFS simulation results are presented below.

A rectangular current conductor with dimensions $10 \times 1 \mathrm{~mm}^{2}$, as the one presented in Figure 6.3 is being tested. Total current $I=600 \mathrm{~A}$ is flowing through the conductor. The magnetic flux density is measured at the conductor surface at several positions and frequencies, as it is presented in Figures 6.4 and 6.5 respectively. The position results in Figure 6.4 are normalized.

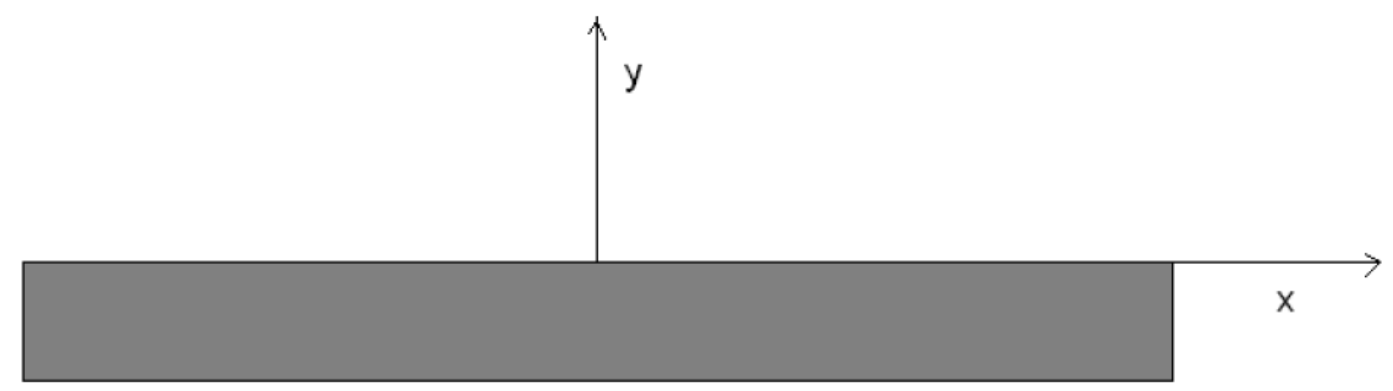

Figure 6.3: Rectangular current conductor
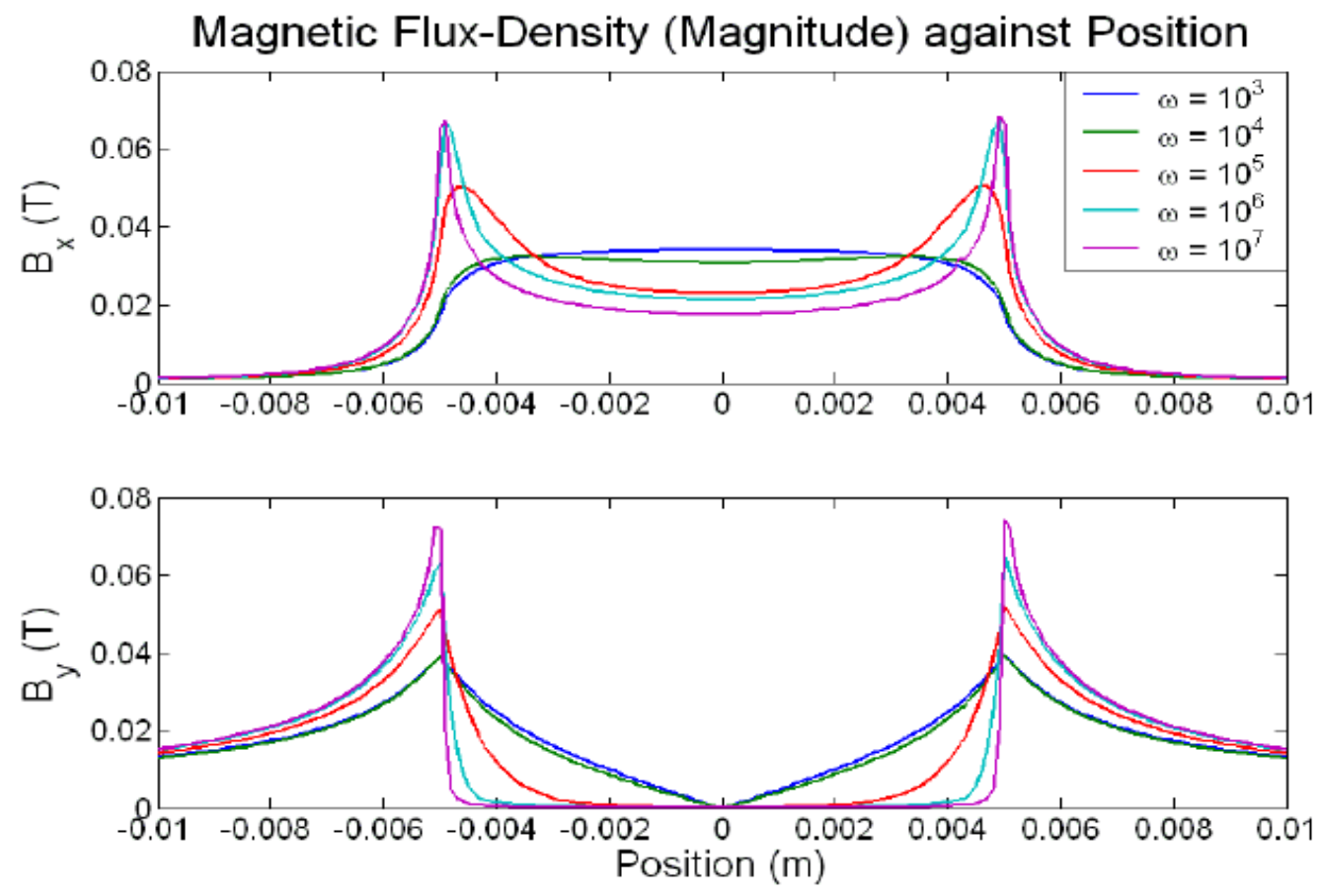

Figure 6.4: Magnetic Flux Density against Position 

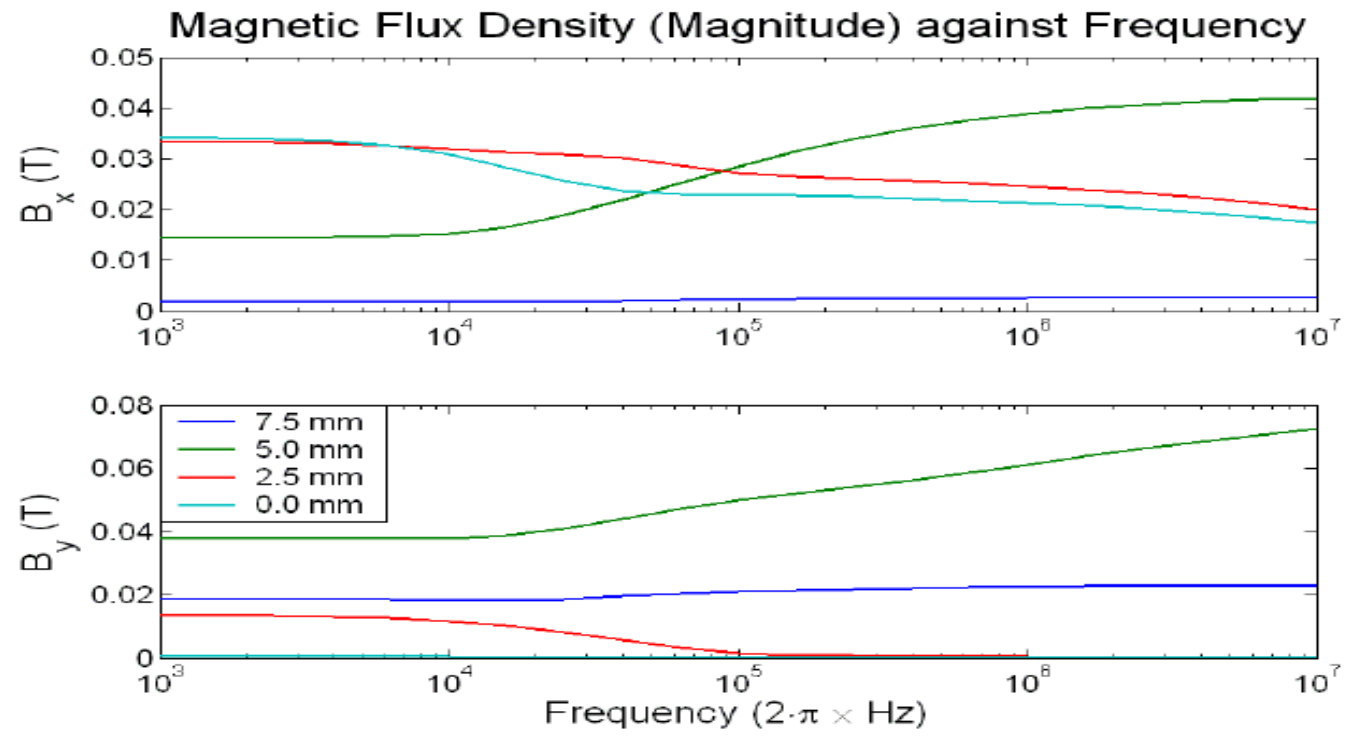

Figure 6.5: Magnetic Flux Density against Frequency

The 2D current conductor (made of copper) with the cross-section presented in Figure 6.6 has been tested as current sensor. Dimensions are stated in Figure 6.6 and measured in millimeters. The total current cross-section area is $S=3.5 \times 10^{-5} \mathrm{~m}^{2}$. Constant current $\mathrm{I}=\mathrm{S} \times$ $\sigma_{\text {copper }}=2100 \mathrm{~A}$ is flowing through the conductor, where $1 / \sigma_{\text {copper }}$ is the resistivity of pure copper. The electric field intensity within the conductor is $E=d V / d x=1 \mathrm{~V} / \mathrm{m}$. The lower part of the conductor serves also as the support of the 2D HALL (vertical and horizontal) pair.

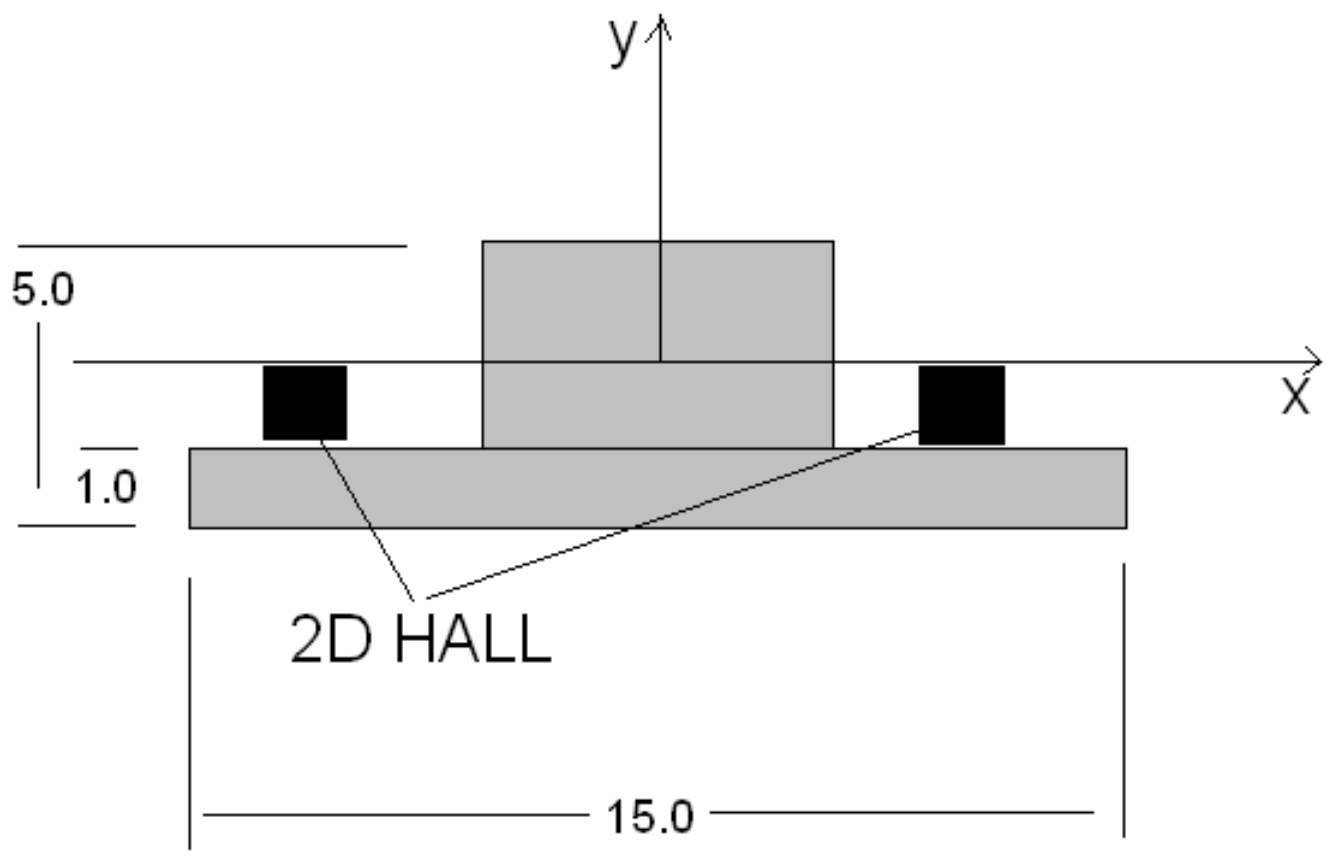

Figure 6.6: Current conductor (made of copper) 
Galvanically Isolated, Wide-Band Current Sensors

MFS Simulation results for the conductor of Figure 6.6 are presented in Figures 6.7 and 6.8, where flux density against position and against frequency is presented. The position results in Figure 6.7 are normalized.
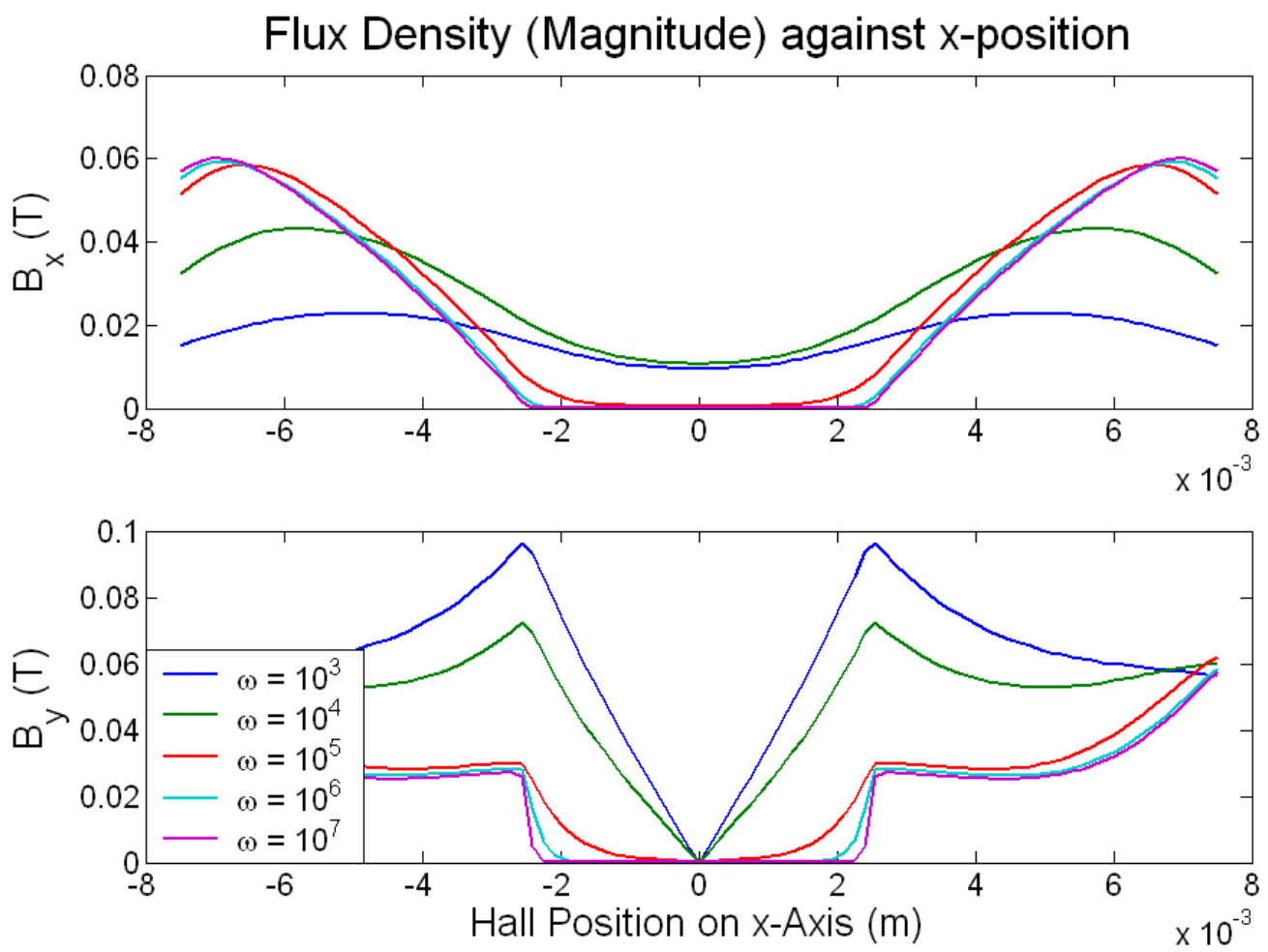

Figure 6.7: Flux Density against $x$ position 
Galvanically Isolated, Wide-Band Current Sensors
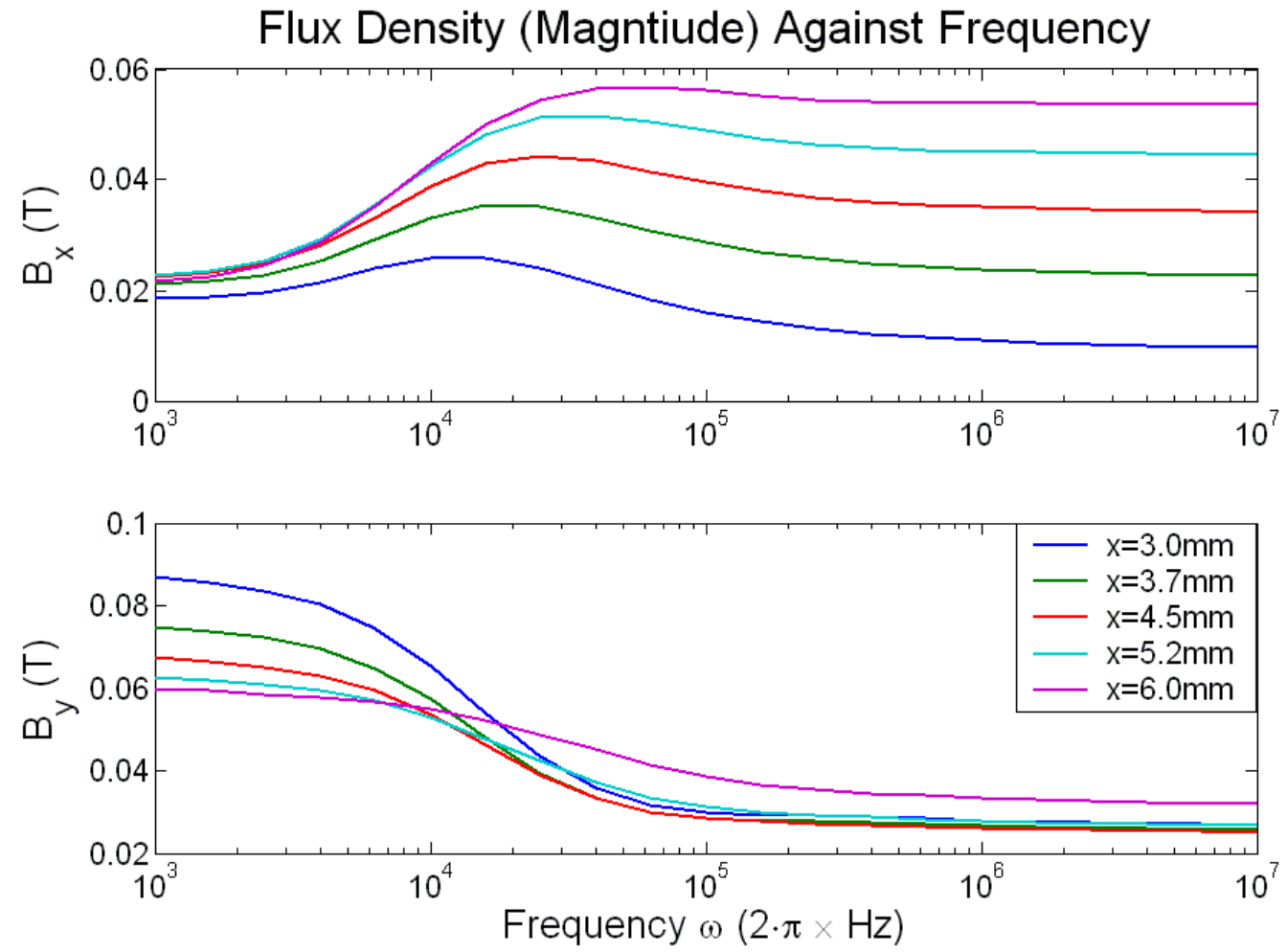

Figure 6.8: Flux Density against Frequency

According to the results that are presented in Figures 6.7 and 6.8 we can assume that a chip with dimensions $2 \times 3 \mathrm{~mm}^{2}$ containing a pair of vertical and horizontal Hall devices that is positioned in a way that the vertical device is at the $\mathrm{x}=6 \mathrm{~mm}$ position, while the horizontal is at the $x=3 \mathrm{~mm}$ position can be used as high frequency current sensor. The vertical device works as a high-pass magnetic filter, while the horizontal one is a low-pass filter. 
Galvanically Isolated, Wide-Band Current Sensors

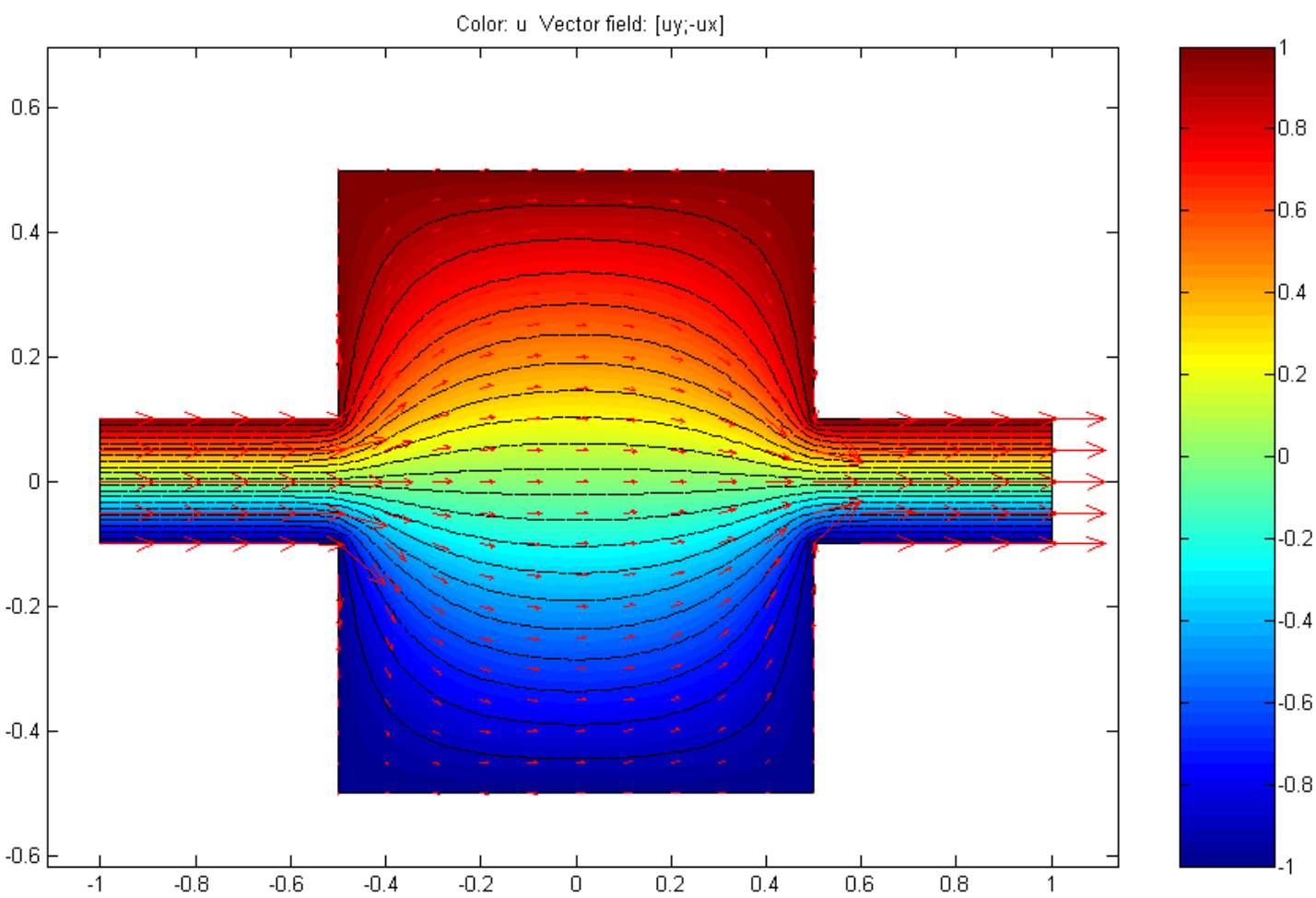

Figure 6.8: Intensity for Low Frequency Current

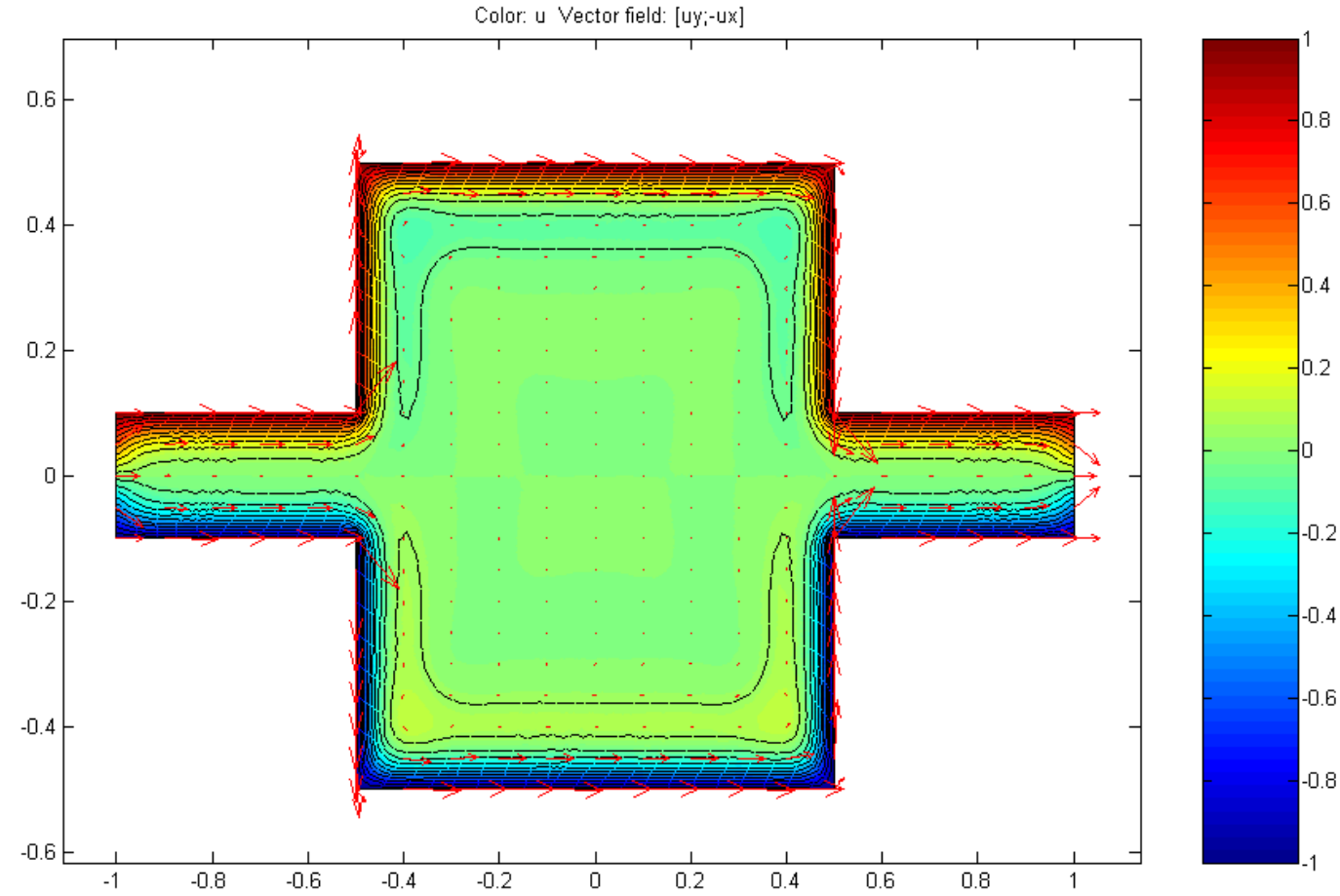

Figure 6.9: Intensity for High Frequency Current

In the above Figures 6.8 and 6.9, MFS simulation results are presented for a device having the shape that is show in the Figures. For the same current conductor, the intensity is presented against position, for low and high frequency current respectively. The position is 
Galvanically Isolated, Wide-Band Current Sensors

normalized. For the conductor of Figures 6.8 and 6.9 , the intensity against position for various frequency values is presented in Figure 6.10. The position here is also normalized.
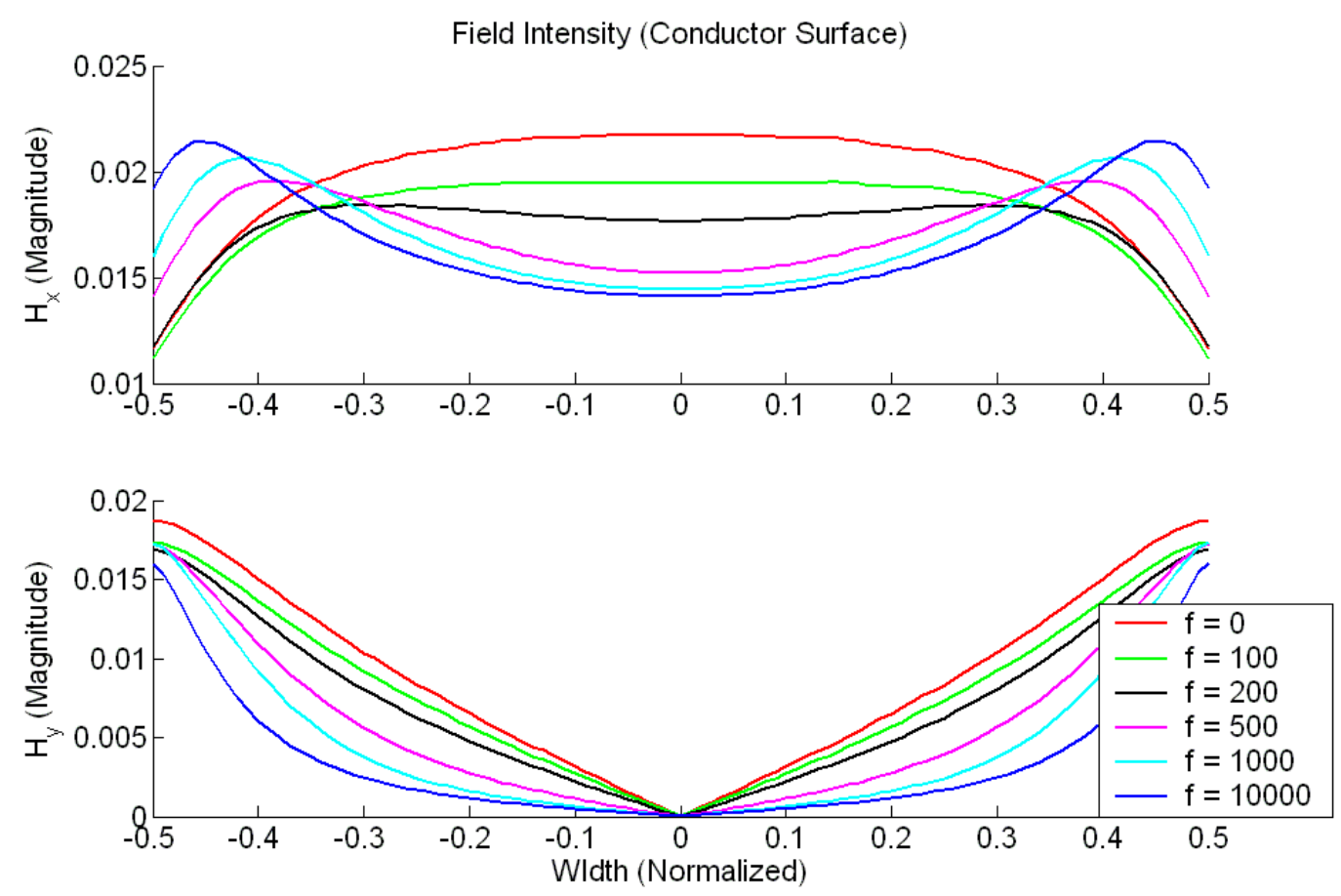

Figure 6.10: Flied intensity at the conductor's surface

According to the results of Figures 6.9 and 6.10, a vertical Hall device at position 0.4 works as a low-pass magnetic filter.

MFS simulation results for the current sensor of Figure 6.2 are presented in Figures 6.11 and 6.12 . 
Galvanically Isolated, Wide-Band Current Sensors

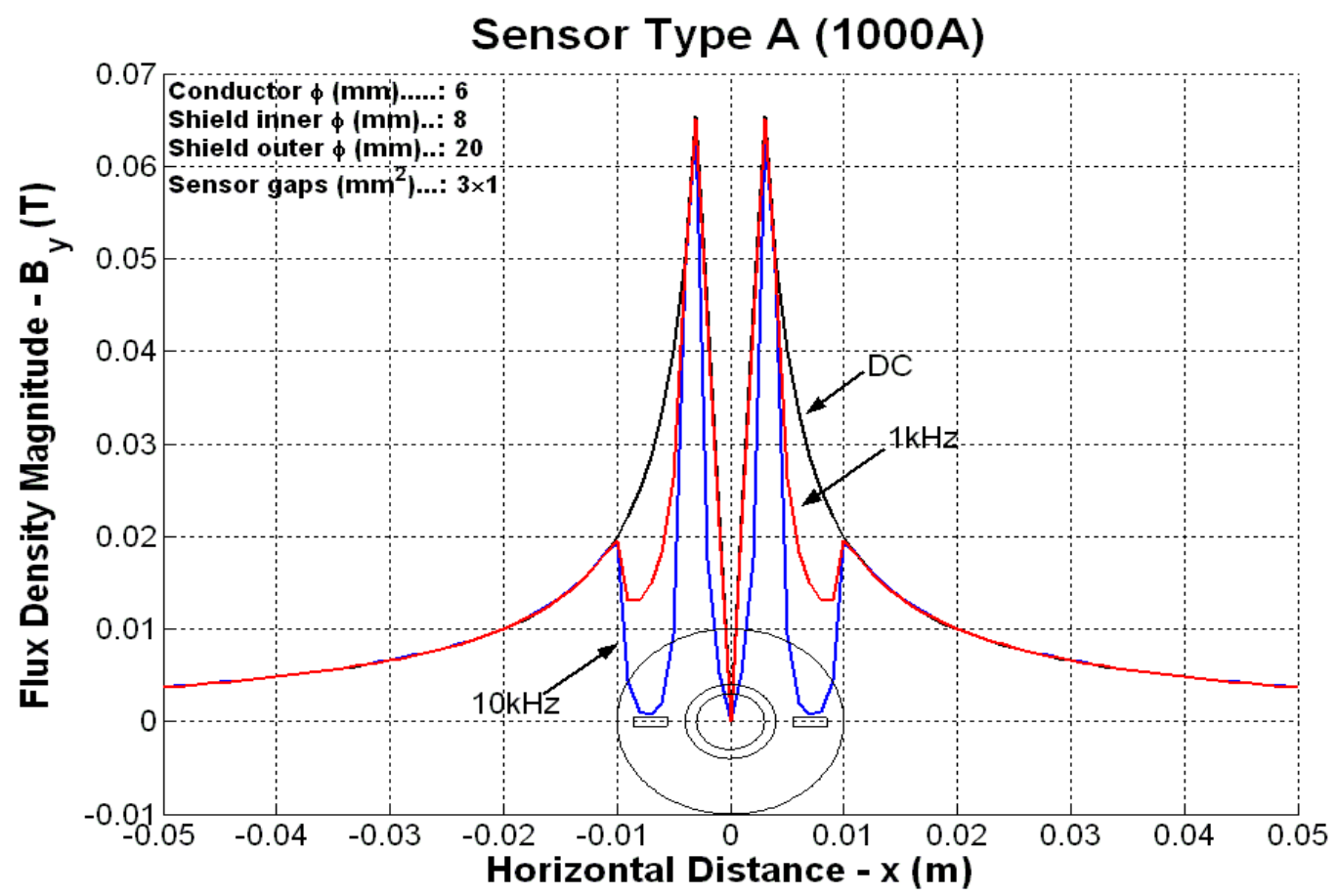

Figure 6.11: Flux Density against $x$ position for various frequency values

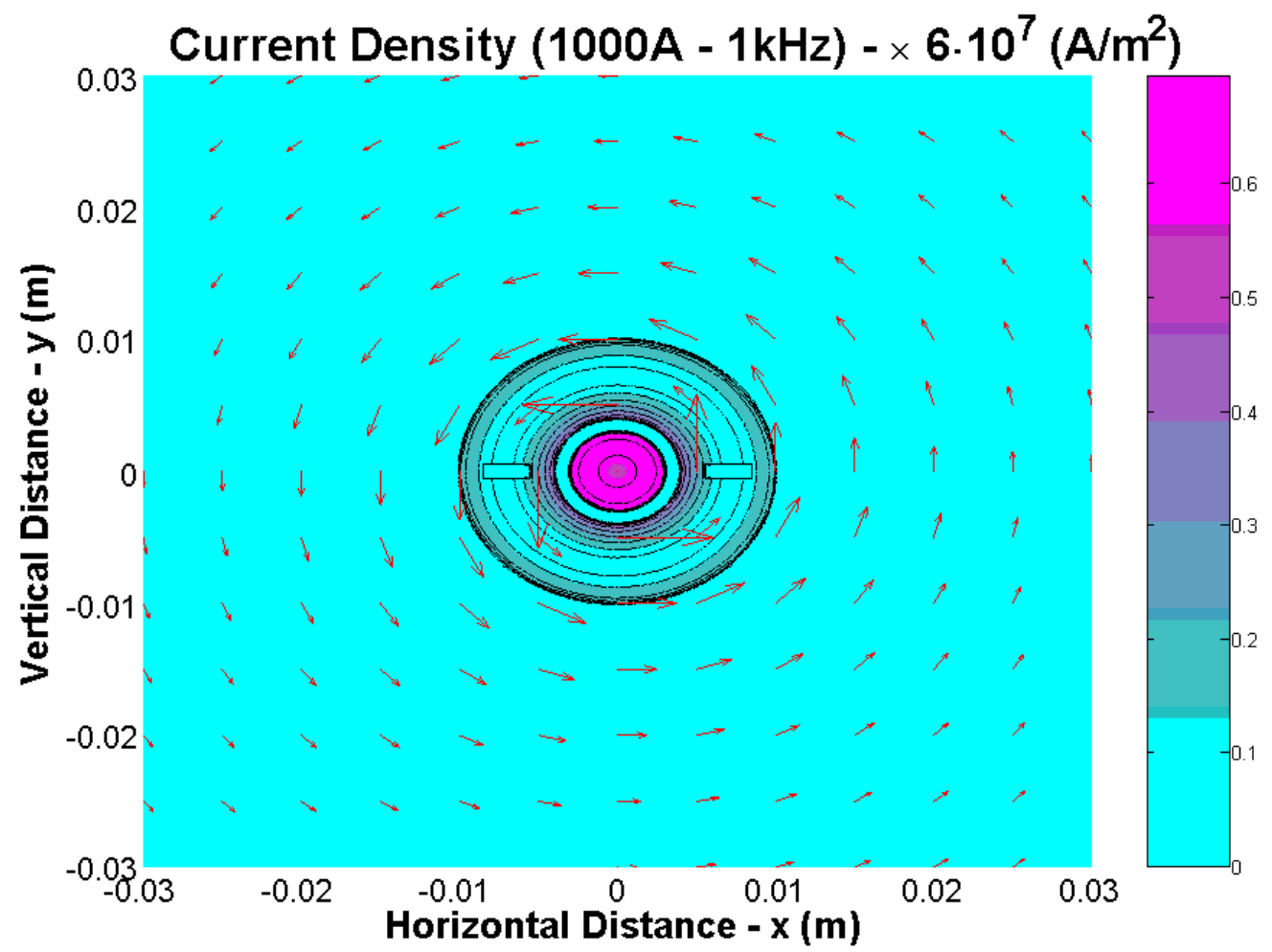

Figure 6.12: Flux Density

According to the simulation results of Figure 6.11 if the Hall sensors are place at $x=-0.005$ and $x=0.005$ positions, these devices work as low-pass filters. 
Galvanically Isolated, Wide-Band Current Sensors

\section{References}

[1] http://www.mathworks.com/products/matlab/

[2] R.S.Popovic, Hall Effect Devices, IOP Publishing, 2004.

[3] R.S.Popovic and B.Halg, Nonlinearity in Hall devices and its compensation, Solid-State Electronics (1988) 1681-1688.

[4] F. Liakou, P. Dimitropoulos, G. Stamoulis, 2D Simulator for Eddy Current Field Filters, Eurosensors XXV, 2011, Athens, Greece

[5] William H. Hayt, John A. Buck, Engineering Electromagnetics, 2000 